\title{
NONLINEAR CONNECTIONS AND SPINOR GEOMETRY
}

\author{
SERGIU I. VACARU and NADEJDA A. VICOL
}

Received 21 December 2002

\begin{abstract}
We present an introduction to the geometry of higher-order vector and covector bundles (including higher-order generalizations of the Finsler geometry and Kaluza-Klein gravity) and review the basic results on Clifford and spinor structures on spaces with generic local anisotropy modeled by anholonomic frames with associated nonlinear connection structures. We emphasize strong arguments for application of Finsler-like geometries in modern string and gravity theory, noncommutative geometry and noncommutative field theory, and gravity.
\end{abstract}

2000 Mathematics Subject Classification: 15A66, 58B20, 53C60, 83C60, 83E15.

1. Introduction. Nowadays, interest has been established in non-Riemannian geometries derived in the low-energy string theory [18, 64, 65], noncommutative geometry $[1,3,8,12,15,22,32,34,53,55,67,109,111,112]$, and quantum groups [33, 35, 36, 37]. Various types of Finsler-like structures can be parametrized by generic off-diagonal metrics, which cannot be diagonalized by coordinate transforms but only by anholonomic maps with associated nonlinear connection (in brief, N-connection). Such structures may be defined as exact solutions of gravitational field equations in the Einstein gravity and its generalizations $[75,79,80,94,95,96,97,98,99,100,102,103,104$, $105,109,110,111]$, for instance, in the metric-affine [19, 23, 56] Riemann-Cartan gravity $[24,25]$. Finsler-like configurations are considered in locally anisotropic thermodynamics, kinetics, related stochastic processes [85, 96, 107, 108], and (super-) string theory $[84,87,90,91,92]$.

The following natural step in these lines is to elucidate the theory of spinors in effectively derived Finsler geometries and to relate this formalism of Clifford structures to noncommutative Finsler geometry. It should be noted that the rigorous definition of spinors for Finsler spaces and generalizations was not a trivial task because (on such spaces) there are no defined even local groups of automorphisms. The problem was solved in $[82,83,88,89,93]$ by adapting the geometric constructions with respect to anholonomic frames with associated N-connection structure. The aim of this work is to outline the geometry of generalized Finsler spinors in a form more oriented to applications in modern mathematical physics.

We start with some historical remarks: the spinors studied by mathematicians and physicists are connected with the general theory of Clifford spaces introduced in 1876 [14]. The theory of spinors and Clifford algebras play a major role in contemporary physics and mathematics. The spinors were discovered by Èlie Cartan in 1913 in 
mathematical form in his researches on representation group theory [10, 11]; he showed that spinors furnish a linear representation of the groups of rotations of a space of arbitrary dimensions. The physicists Pauli [60] and Dirac [20] (in 1927, resp., for the three-dimensional and four-dimensional space-times) introduced spinors for the representation of the wave functions. In general relativity theory spinors and the Dirac equations on (pseudo-) Riemannian spaces were defined in 1929 by Weyl [113], Fock [21], and Schrödinger [68]. The books [61, 62, 63] by Penrose and Rindler summarize the spinor and twistor methods in space-time geometry (see additional references [7, 9, 26, 27, 31, 54] on Clifford structures and spinor theory).

Spinor variables were introduced in Finsler geometries by Takano in [73] where he dismissed anisotropic dependencies not only on vectors on the tangent bundle but also on some spinor variables in a spinor bundle on a space-time manifold. Then generalized Finsler geometries, with spinor variables, were developed by Ono and Takano in a series of publications during 1990-1993 [57, 58, 59, 74]. The next steps were investigations of anisotropic and deformed geometries with spinor and vector variables and applications in gauge and gravity theories elaborated by Stavrinos and his students, Koutroubis, Manouselis, and Balan at the beginning of 1994 [69, 70, 71, 72]. In those works the authors assumed that some spinor variables may be introduced in a Finsler-like way, but they did not relate the Finlser metric to a Clifford structure and restricted the spinor-gauge Finsler constructions only to antisymmetric spinor metrics on two-spinor fibers with possible generalizations to four-dimensional Dirac spinors.

Isotopic spinors, related with SU(2) internal structural groups, were considered in generalized Finsler gravity and gauge theories also by Asanov and Ponomarenko [4]. In that book, and in other papers on Finsler geometry with spinor variables, the authors did not investigate the possibility of introducing a rigorous mathematical definition of spinors on spaces with generic local anisotropy.

An alternative approach to spinor differential geometry and generalized Finsler spaces was elaborated, at the beginning of 1994, in a series of papers and communications by Vacaru and coauthors [83, 88, 101]. This direction originates from Clifford algebras, Clifford bundles [28], Penrose's spinor, and twistor space-time geometry $[61,62,63]$, which were reconsidered for the case of nearly autoparallel maps (generalized conformal transforms) in [86]. In the works [82, 83, 88, 89], a rigorous definition of spinors for Finsler spaces, and their generalizations, was given. It was proven that a Finsler, or Lagrange, metric (in a tangent or, more generally, in a vector bundle) induces naturally a distinguished Clifford (spinor) structure which is locally adapted to the nonlinear connection structure. Such spinor spaces could be defined for arbitrary dimensions of base and fiber subspaces, their spinor metrics are symmetric, antisymmetric, or nonsymmetric, depending on the corresponding base and fiber dimensions. That work resulted in the formation of the spinor differential geometry of generalized Finsler spaces and developed a number of geometric applications to the theory of gravitational and matter field interactions with generic local anisotropy.

The geometry of anisotropic spinors and (distinguished by nonlinear connections) Clifford structures was elaborated for higher-order anisotropic spaces [82, 83, 92, 93] and, more recently, for Hamilton and Lagrange spaces [109, 111]. 
We emphasize that the theory of anisotropic spinors may be related not only to generalized Finsler, Lagrange, Cartan, and Hamilton spaces or their higher-order generalizations, but also to anholonomic frames with associated nonlinear connections which appear naturally even in (pseudo-) Riemannian and Riemann-Cartan geometries if off-diagonal metrics are considered [94, 96, 97, 98, 102, 103, 104, 105, 110]. In order to construct exact solutions of the Einstein equations in general relativity and extradimensional gravity (for lower dimensions see [85, 96, 107, 108]), it is more convenient to diagonalize space-time metrics by using some anholonomic transforms. As a result, one induces locally anisotropic structures on space-time, which are related to anholonomic (anisotropic) spinor structures.

The main purpose of the present review is to present a detailed summary and new results on spinor differential geometry for generalized Finsler spaces and (pseudo-) Riemannian space-times provided with an anholonomic frame and associated nonlinear connection structure, to discuss and compare the existing approaches and to consider applications to modern gravity and gauge theories. The work is based on communications [109, 111].

2. (Co-) vector bundles and N-connections. We outline the basic definitions and denotations for the vector and tangent (and their dual spaces) bundles and higher-order vector/covector bundle geometry. In this work, we consider that the space-time geometry can be modeled both on a (pseudo-) Riemannian manifold $V^{[n+m]}$ of dimension $n+m$ and on a vector bundle (or its dual, covector bundle) being, for simplicity, locally trivial with a base space $M$ of dimension $n$ and a typical fiber $F$ (cofiber $F^{*}$ ) of dimension $m$, or as a higher-order extended vector/covector bundle (we follow the geometric constructions and definitions of $[45,46,47,48,49,50,51,52]$, which were generalized for vector superbundles in [90, 91, 92]). Such (pseudo-) Riemannian spaces and/or vector/covector bundles enabled with compatible fibered and/or anholonomic structures are called anisotropic space-times. If the anholonomic structure with associated nonlinear connection is modeled on higher-order vector/covector bundles, we use the term higher-order anisotropic space-time. In this section, we usually omit proofs which can be found in the mentioned monographs [45, 46, 47, 48, 49, 50, 51, 52, 92].

2.1. (Co-) vector and tangent bundles. A locally trivial vector bundle, in brief, $v$ bundle, $\mathscr{E}=(E, \pi, M, G r, F)$ is introduced as a set of spaces and surjective map with the properties that a real vector space $F=\mathbb{R}^{m}$ of dimension $m(\operatorname{dim} F=m, \mathbb{R}$ denotes the real numbers field) defines the typical fiber, the structural group is chosen to be the group of automorphisms of $\mathbb{R}^{m}$, that is, $G r=\mathrm{GL}(m, \mathbb{R})$, and $\pi: E \rightarrow M$ is a differentiable surjection of a differentiable manifold $E$ (total space, $\operatorname{dim} E=n+m$ ) to a differentiable manifold $M$ (base space, $\operatorname{dim} M=n$ ). The local coordinates on $\mathscr{E}$ are denoted $u^{\alpha}=$ $\left(x^{i}, y^{a}\right)$, or in brief $u=(x, y)$ (the Latin indices $i, j, k, \ldots=1,2, \ldots, n$ define coordinates of geometrical objects with respect to a local frame on base space $M$; the Latin indices $a, b, c, \ldots=1,2, \ldots, m$ define fiber coordinates of geometrical objects and the Greek indices $\alpha, \beta, \gamma, \ldots$ are considered as cumulative ones for coordinates of objects defined on the total space of a v-bundle). 
Coordinate transforms $u^{\alpha^{\prime}}=u^{\alpha^{\prime}}\left(u^{\alpha}\right)$ on a v-bundle $\mathscr{E}$ are defined as $\left(x^{i}, y^{a}\right) \rightarrow\left(x^{i^{\prime}}\right.$, $\left.y^{a^{\prime}}\right)$, where

$$
x^{i^{\prime}}=x^{i^{\prime}}\left(x^{i}\right), \quad y^{a^{\prime}}=K_{a}^{a^{\prime}}\left(x^{i}\right) y^{a},
$$

and matrix $K_{a}^{a^{\prime}}\left(x^{i}\right) \in \mathrm{GL}(m, \mathbb{R})$ are functions of a necessary smoothness class.

A local coordinate parametrization of v-bundle $\mathscr{E}$ naturally defines a coordinate basis

$$
\partial_{\alpha}=\frac{\partial}{\partial u^{\alpha}}=\left(\partial_{i}=\frac{\partial}{\partial x^{i}}, \partial_{a}=\frac{\partial}{\partial y^{a}}\right)
$$

and the reciprocal to (2.2) coordinate basis

$$
d^{\alpha}=d u^{\alpha}=\left(d^{i}=d x^{i}, d^{a}=d y^{a}\right)
$$

which is uniquely defined from the equations $d^{\alpha} \circ \partial_{\beta}=\delta_{\beta}^{\alpha}$, where $\delta_{\beta}^{\alpha}$ is the Kronecker symbol and by "。” we denote the inner (scalar) product in the tangent bundle $\mathcal{T}_{\mathscr{E}}$.

A tangent bundle (in brief, $t$-bundle) $(T M, \pi, M)$ to a manifold $M$ can be defined as a particular case of a v-bundle when the dimensions of the base and fiber spaces (the last one considered as the tangent subspace) are identical, $n=m$. In this case both types of indices $i, k, \ldots$ and $a, b, \ldots$ take the same values $1,2, \ldots, n$. For t-bundles, the matrices of fiber coordinates transforms from (2.1) can be written as $K_{i}^{i^{\prime}}=\partial x^{i^{\prime}} / \partial x^{i}$.

We will also use the concept of covector bundle (in brief, $c$-bundles) $\breve{\mathscr{E}}=\left(\breve{E}, \pi^{*}, M\right.$, $\left.G r, F^{*}\right)$ which is introduced as a dual vector bundle for which the typical fiber $F^{*}$ (cofiber) is considered to be the dual vector space (covector space) to the vector space $F$. The fiber coordinates $p_{a}$ of $\breve{E}$ are dual to $y^{a}$ in $E$. The local coordinates on total space $\breve{E}$ are denoted $\breve{u}=(x, p)=\left(x^{i}, p_{a}\right)$. The coordinate transforms on $\breve{E}, \breve{u}=\left(x^{i}, p_{a}\right) \rightarrow \breve{u}^{\prime}=$ $\left(x^{i^{\prime}}, p_{a^{\prime}}\right)$, are written as

$$
x^{i^{\prime}}=x^{i^{\prime}}\left(x^{i}\right), \quad p_{a^{\prime}}=K_{a^{\prime}}^{a}\left(x^{i}\right) p_{a} .
$$

The coordinate bases on $E^{*}$ are denoted

$$
\breve{\partial}_{\alpha}=\frac{\breve{\partial}}{\partial u^{\alpha}}=\left(\partial_{i}=\frac{\partial}{\partial x^{i}}, \breve{\partial}^{a}=\frac{\breve{\partial}}{\partial p_{a}}\right), \quad \breve{d}^{\alpha}=\breve{d} u^{\alpha}=\left(d^{i}=d x^{i}, \breve{d}_{a}=d p_{a}\right) .
$$

We use breve symbols in order to distinguish the geometrical objects on a cv-bundle $\mathscr{E}^{*}$ from those on a v-bundle $\mathscr{E}$.

As a particular case with the same dimension of base space and cofiber, one obtains the cotangent bundle $\left(T^{*} M, \pi^{*}, M\right)$, in brief, ct-bundle, being dual to $T M$. The fibre coordinates $p_{i}$ of $T^{*} M$ are dual to $y^{i}$ in $T M$. The coordinate transforms (2.4) on $T^{*} M$ are stated by some matrices $K_{k^{\prime}}^{k}\left(x^{i}\right)=\partial x^{k} / \partial x^{k^{\prime}}$.

In our further considerations, we will distinguish the base and cofiber indices.

2.2. Higher-order (co-) vector bundles. The geometry of higher-order tangent and cotangent bundles provided with a nonlinear connection structure was elaborated in $[45,49,50,51,52]$ in order to geometrize the higher-order Lagrange and Hamilton mechanics. In this case we have base spaces and fibers of the same dimension. To 
develop the approach to modern high-energy physics (in superstring and Kaluza-Klein theories), we introduced (in $[82,83,90,91,92,93]$ ) the concept of higher-order vector bundle with the fibers consisting of finite "shells" of vector, or covector, spaces of different dimensions not obligatorily coinciding with the base space dimension.

Definition 2.1. A distinguished vector/covector space, in brief, dvc-space, of type

$$
\tilde{F}=F[v(1), v(2), c v(3), \ldots, c v(z-1), v(z)]
$$

is a vector space decomposed into an invariant oriented direct sum

$$
\tilde{F}=F_{(1)} \oplus F_{(2)} \oplus F_{(3)}^{*} \oplus \cdots \oplus F_{(z-1)}^{*} \oplus F_{(z)}
$$

of vector spaces $F_{(1)}, F_{(2)}, \ldots, F_{(z)}$ of respective dimensions

$$
\operatorname{dim} F_{(1)}=m_{1}, \operatorname{dim} F_{(2)}=m_{2}, \ldots, \operatorname{dim} F_{(z)}=m_{z},
$$

and of covector spaces $F_{(3)}^{*}, \ldots, F_{(z-1)}^{*}$ of respective dimensions

$$
\operatorname{dim} F_{(3)}^{*}=m_{3}^{*}, \ldots, \operatorname{dim} F_{(z-1)}^{*}=m_{(z-1)}^{*} .
$$

As a particular case, we obtain a distinguished vector space, in brief dv-space (resp., a distinguished covector space, in brief dcv-space), if all components of the sum are vector (resp., covector) spaces. We note that we have fixed, for simplicity, an orientation of (co-) vector subspaces like in (2.6).

Coordinates on $\tilde{F}$ are denoted

$$
\tilde{y}=\left(y_{(1)}, y_{(2)}, p_{(3)}, \ldots, p_{(z-1)}, y_{(z)}\right)=\left\{y^{\left\langle\alpha_{z}\right\rangle}\right\}=\left(y^{a_{1}}, y^{a_{2}}, p_{a_{3}}, \ldots, p_{a_{z-1}}, y^{a_{z}}\right),
$$

where indices run correspondingly to the values $a_{1}=1,2, \ldots, m_{1} ; a_{2}=1,2, \ldots, m_{2} ; \ldots$; $a_{z}=1,2, \ldots, m_{z}$.

DEFINITION 2.2. A higher-order vector/covector bundle, in brief, hvc-bundle, of type $\tilde{\mathscr{E}}=\tilde{\mathscr{E}}[v(1), v(2), c v(3), \ldots, c v(z-1), v(z)]$ is a vector bundle $\tilde{\mathscr{E}}=\left(\tilde{E}, p^{\langle d\rangle}, \tilde{F}, M\right)$ with corresponding total, $\tilde{E}$, and base, $M$, spaces, surjective projection $p^{\langle d\rangle}: \tilde{E} \rightarrow M$, and typical fiber $\tilde{F}$.

We define the higher-order vector (resp., covector) bundles, in brief, hv-bundles (resp., in brief, hcv-bundles), if the typical fibre is a dv-space (resp., a dcv-space) as particular cases of the hvc-bundles.

An hvc-bundle is constructed as an oriented set of enveloping "shell-by-shell" vbundles and/or cv-bundles,

$$
p^{\langle s\rangle}: \tilde{E}^{\langle s\rangle} \longrightarrow \tilde{E}^{\langle s-1\rangle}
$$

where we use the index $\langle s\rangle=0,1,2, \ldots, z$ in order to enumerate the shells, when $\tilde{E}^{\langle 0\rangle}=$ $M$. Local coordinates on $\tilde{E}^{\langle s\rangle}$ are denoted

$$
\tilde{u}_{(s)}=\left(x, \tilde{y}_{\langle s\rangle}\right)=\left(x, y_{(1)}, y_{(2)}, p_{(3)}, \ldots, y_{(s)}\right)=\left(x^{i}, y^{a_{1}}, y^{a_{2}}, p_{a_{3}}, \ldots, y^{a_{s}}\right) .
$$


If $\langle s\rangle=\langle z\rangle$, we obtain a complete coordinate system on $\tilde{\mathscr{E}}$ denoted in brief

$$
\tilde{u}=(x, \tilde{y})=\tilde{u}^{\alpha}=\left(x^{i}=y^{a_{0}}, y^{a_{1}}, y^{a_{2}}, p_{a_{3}}, \ldots, p_{a_{z-1}}, y^{a_{z}}\right) .
$$

We will use the general commutative indices $\alpha, \beta, \ldots$ for objects on hvc-bundles which are marked by tilde, like $\tilde{u}, \tilde{u}^{\alpha}, \ldots, \tilde{E}^{\langle s\rangle}, \ldots$.

The coordinate bases on $\tilde{\mathscr{E}}$ are denoted

$$
\begin{aligned}
& \tilde{\partial}_{\alpha}=\frac{\tilde{\partial}}{\partial u^{\alpha}}=\left(\partial_{i}=\frac{\partial}{\partial x^{i}}, \partial_{a_{1}}=\frac{\partial}{\partial y^{a_{1}}}, \partial_{a_{2}}=\frac{\partial}{\partial y^{a_{2}}}, \breve{\partial}^{a_{3}}=\frac{\breve{\partial}}{\partial p_{a_{3}}}, \ldots, \partial_{a_{z}}=\frac{\partial}{\partial y^{a_{z}}}\right), \\
& \tilde{d}^{\alpha}=\tilde{d} u^{\alpha}=\left(d^{i}=d x^{i}, d^{a_{1}}=d y^{a_{1}}, d^{a_{2}}=d y^{a_{2}}, \breve{d}_{a_{3}}=d p_{a_{3}}, \ldots, d^{a_{z}}=d y^{a_{z}}\right) .
\end{aligned}
$$

We give two examples of higher-order tangent/cotangent bundles (when the dimensions of fibers/cofibers coincide with the dimension of bundle space, see [45, 49, 50, $51,52])$.

2.2.1. Osculator bundle. The $k$-osculator bundle is identified with the $k$-tangent bundle $\left(T^{k} M, p^{(k)}, M\right)$ of an $n$-dimensional manifold $M$. We denote the local coordinates $\tilde{u}^{\alpha}=\left(x^{i}, y_{(1)}^{i}, \ldots, y_{(k)}^{i}\right)$, where we have identified $y_{(1)}^{i} \simeq y^{a_{1}}, \ldots, y_{(k)}^{i} \simeq y^{a_{k}}, k=z$, in order to have similarity with denotations from $[45,49,50,51,52]$. The coordinate transforms $\tilde{u}^{\alpha^{\prime}} \rightarrow \tilde{u}^{\alpha^{\prime}}\left(\tilde{u}^{\alpha}\right)$ preserving the structure of such higher-order vector bundles are parametrized:

$$
\begin{aligned}
x^{i^{\prime}} & =x^{i^{\prime}}\left(x^{i}\right), \quad \operatorname{det}\left(\frac{\partial x^{i^{\prime}}}{\partial x^{i}}\right) \neq 0, \\
y_{(1)}^{i^{\prime}} & =\frac{\partial x^{i^{\prime}}}{\partial x^{i}} y_{(1)}^{i}, \\
2 y_{(2)}^{i^{\prime}} & =\frac{\partial y_{(1)}^{i^{\prime}}}{\partial x^{i}} y_{(1)}^{i}+2 \frac{\partial y_{(1)}^{i^{\prime}}}{\partial y^{i}} y_{(2)}^{i}, \\
& \vdots \\
k y_{(k)}^{i^{\prime}} & =\frac{\partial y_{(1)}^{i^{\prime}}}{\partial x^{i}} y_{(1)}^{i}+\cdots+k \frac{\partial y_{(k-1)}^{i^{\prime}}}{\partial y_{(k-1)}^{i}} y_{(k)}^{i},
\end{aligned}
$$

where the equalities

$$
\frac{\partial y_{(s)}^{i^{\prime}}}{\partial x^{i}}=\frac{\partial y_{(s+1)}^{i^{\prime}}}{\partial y_{(1)}^{i}}=\cdots=\frac{\partial y_{(k)}^{i^{\prime}}}{\partial y_{(k-s)}^{i}}
$$

hold for $s=0, \ldots, k-1$ and $y_{(0)}^{i}=x^{i}$.

The natural coordinate frame on $\left(T^{k} M, p^{(k)}, M\right)$ is defined by $\tilde{\partial}_{\alpha}=\left(\partial / \partial x^{i}, \partial / \partial y_{(1)}^{i}, \ldots\right.$, $\left.\partial / \partial y_{(k)}^{i}\right)$ and the coframe is $\tilde{d}_{\alpha}=\left(d x^{i}, d y_{(1)}^{i}, \ldots, d y_{(k)}^{i}\right)$. These formulas are, respectively, some particular cases of (2.14). 
2.2.2. The dual bundle of $k$-osculator bundle. This higher-order vector/covector bundle, denoted as $\left(T^{* k} M, p^{* k}, M\right)$, is defined as the dual bundle to the $k$-tangent bundle $\left(T^{k} M, p^{k}, M\right)$. The local coordinates (parametrized as in the previous paragraph) are

$$
\tilde{u}=\left(x, y_{(1)}, \ldots, y_{(k-1)}, p\right)=\left(x^{i}, y_{(1)}^{i}, \ldots, y_{(k-1)}^{i}, p_{i}\right) \in T^{* k} M
$$

The coordinate transforms on $\left(T^{* k} M, p^{* k}, M\right)$ are

$$
\begin{aligned}
x^{i^{\prime}} & =x^{i^{\prime}}\left(x^{i}\right), \quad \operatorname{det}\left(\frac{\partial x^{i^{\prime}}}{\partial x^{i}}\right) \neq 0, \\
y_{(1)}^{i^{\prime}} & =\frac{\partial x^{i^{\prime}}}{\partial x^{i}} y_{(1)}^{i}, \\
2 y_{(2)}^{i^{\prime}} & =\frac{\partial y_{(1)}^{i^{\prime}}}{\partial x^{i}} y_{(1)}^{i}+2 \frac{\partial y_{(1)}^{i^{\prime}}}{\partial y^{i}} y_{(2)}^{i}, \\
& \vdots \\
(k-1) y_{(k-1)}^{i^{\prime}} & =\frac{\partial y_{(k-2)}^{i^{\prime}}}{\partial x^{i}} y_{(1)}^{i}+\cdots+k \frac{\partial y_{(k-1)}^{i^{\prime}}}{\partial y_{(k-2)}^{i}} y_{(k-1)}^{i}, \quad p_{i^{\prime}}=\frac{\partial x^{i}}{\partial x^{i^{\prime}}} p_{i},
\end{aligned}
$$

where the equalities

$$
\frac{\partial y_{(s)}^{i^{\prime}}}{\partial x^{i}}=\frac{\partial y_{(s+1)}^{i^{\prime}}}{\partial y_{(1)}^{i}}=\cdots=\frac{\partial y_{(k-1)}^{i^{\prime}}}{\partial y_{(k-1-s)}^{i}}
$$

hold for $s=0, \ldots, k-2$ and $y_{(0)}^{i}=x^{i}$.

The natural coordinate frame on $\left(T^{* k} M, p^{*(k)}, M\right)$ is written in the form $\tilde{\partial}_{\alpha}=\left(\partial / \partial x^{i}\right.$, $\left.\partial / \partial y_{(1)}^{i}, \ldots, \partial / \partial y_{(k-1)}^{i}, \partial / \partial p_{i}\right)$ and the coframe is written as $\tilde{d}_{\alpha}=\left(d x^{i}, d y_{(1)}^{i}, \ldots, d y_{(k-1)}^{i}\right.$, $\left.d p_{i}\right)$. These formulas are, respectively, certain particular cases of (2.14).

2.3. Nonlinear connections. The concept of nonlinear connection, in brief, N-connection, is fundamental in the geometry of vector bundles and anisotropic spaces (see a detailed study and basic references in [46, 47, 48] and, for supersymmetric and/or spinor bundles, see $[90,91,92,106])$. A rigorous mathematical definition is possible by using the formalism of exact sequences of vector bundles.

2.3.1. N-connections in vector bundles. Let $\mathscr{E}=(E, p, M)$ be a v-bundle with typical fiber $\mathbb{R}^{m}$ and $\pi^{T}: T E \rightarrow T M$ being the differential of the map $P$ which is a fibrepreserving morphism of the tangent bundle $\left(T E, \tau_{E}, E\right) \rightarrow E$ and of tangent bundle $(T M, \tau, M) \rightarrow M$. The kernel of the vector bundle morphism, denoted as $\left(V E, \tau_{V}, E\right)$, is called the vertical subbundle over $E$, which is a vector subbundle of the vector bundle $\left(T E, \tau_{E}, E\right)$.

A vector $X_{u}$ tangent to a point $u \in E$ is locally written as $(x, y, X, Y)=\left(x^{i}, y^{a}, X^{i}, Y^{a}\right)$, where the coordinates $\left(X^{i}, Y^{a}\right)$ are defined by the equality $X_{u}=X^{i} \partial_{i}+Y^{a} \partial_{a}$. We have $\pi^{T}(x, y, X, Y)=(x, X)$. Thus the submanifold $V E$ contains the elements which are locally represented as $(x, y, 0, Y)$. 
DEFINITION 2.3. A nonlinear connection $\mathbf{N}$ in a vector bundle $\mathscr{E}=(E, \pi, M)$ is the splitting on the left of the exact sequence

$$
0 \longmapsto V E \longmapsto T E \longmapsto T E / V E \longmapsto 0
$$

where $T E / V E$ is the factor bundle.

By Definition 2.3 a morphism of vector bundles $C: T E \rightarrow V E$ is defined such that the superposition of maps $C \circ i$ is the identity on $V E$, where $i: V E \mapsto V E$. The kernel of the morphism $C$ is a vector subbundle of $\left(T E, \tau_{E}, E\right)$, which is the horizontal subbundle, denoted by $\left(H E, \tau_{H}, E\right)$. Consequently, we can prove that in a v-bundle $\mathscr{E}$, an $\mathrm{N}$-connection can be introduced as a distribution

$$
\left\{N: E_{u} \longrightarrow H_{u} E, T_{u} E=H_{u} E \oplus V_{u} E\right\}
$$

for every point $u \in E$ defining a global decomposition, as a Whitney sum, into horizontal, $H_{\mathscr{E}}^{\mathscr{E}}$, and vertical, $V_{\mathscr{E}}^{\mathscr{E}}$, subbundles of the tangent bundle $T_{\mathscr{E}}^{\mathscr{E}}$ :

$$
T_{\mathscr{E}}=H \mathscr{E} \oplus V_{\mathscr{E}}
$$

Locally, an N-connection in a v-bundle $\mathscr{E}$ is given by its coefficients $N_{i}^{a}(u)=N_{i}^{a}(x, y)$ with respect to bases (2.2) and (2.3), $\mathbf{N}=N_{i}^{a}(u) d^{i} \otimes \partial_{a}$. We note that a linear connection in a v-bundle $\mathscr{E}$ can be considered as a particular case of an N-connection when $N_{i}^{a}(x, y)=K_{b i}^{a}(x) y^{b}$, where functions $K_{a i}^{b}(x)$ on the base $M$ are called the Christoffel coefficients.

2.3.2. N-connections in covector bundles. A nonlinear connection in a cv-bundle $\breve{\mathscr{E}}$ (in brief an $\check{\mathrm{N}}$-connection) can be introduced in a similar fashion as for v-bundles by reconsidering the corresponding definitions for cv-bundles. For instance, it may be defined by a Whitney decomposition, into horizontal, $H \check{\mathscr{C}}$, and vertical, $V_{\mathscr{\mathscr { E }}}^{\breve{6}}$, subbundles of the tangent bundle $T_{\mathscr{E}}^{\breve{E}}$ :

$$
T \breve{\mathscr{E}}=H \breve{\mathscr{E}} \oplus V \breve{\mathscr{E}} .
$$

Hereafter, for the sake of brevity, we will omit details on the definition of geometrical objects on cv-bundles if they are very similar to those for v-bundles: we will present only the basic formulas by emphasizing the most important common points and differences.

DEFINITION 2.4. An $\check{N}$-connection on $\breve{\mathscr{E}}$ is a differentiable distribution

$$
\breve{N}: \breve{\mathscr{E}} \longrightarrow \breve{N}_{u} \in T_{u}^{*} \breve{\mathscr{E}}
$$

which is supplementary to the vertical distribution $V$, that is, $T_{u} \breve{\mathscr{E}}=\breve{N}_{u} \oplus \breve{V}_{u}$, for all $\breve{\mathscr{E}}$.

The same definition is true for $\check{\mathrm{N}}$-connections in ct-bundles, we have to change in Definition 2.4 the symbol $\breve{\mathscr{E}}$ to $T^{*} M$.

An $\check{\mathrm{N}}$-connection in a cv-bundle $\breve{\mathscr{C}}$ is given locally by its coefficients $\breve{N}_{i a}(u)=\breve{N}_{i a}(x, p)$ with respect to bases (2.2) and (2.3), $\breve{\mathrm{N}}=\breve{N}_{i a}(u) d^{i} \otimes \breve{\partial} a$. 
We emphasize that if an N-connection is introduced in a v-bundle (resp., cv-bundle), we have to adapt the geometric constructions to the N-connection structure (resp., the Ň-connection structure).

2.3.3. N-connections in higher-order bundles. The concept of N-connection can be defined for a higher-order vector/covector bundle in a standard manner like in the usual vector bundles.

DEFINITION 2.5. A nonlinear connection $\tilde{N}$ in hvc-bundle

$$
\tilde{\mathscr{E}}=\tilde{\mathscr{E}}[v(1), v(2), c v(3), \ldots, c v(z-1), v(z)]
$$

is a splitting of the left of the exact sequence

$$
0 \longrightarrow V \tilde{\mathscr{E}} \longrightarrow T^{\tilde{\mathscr{E}}} \longrightarrow T^{\tilde{\mathscr{E}}} / V_{\mathscr{E}}^{\tilde{\mathscr{E}}} \longrightarrow 0
$$

We can associate sequences of type (2.26) to every mapping of intermediary subbundles. For simplicity, we present here the Whitney decomposition

$$
T_{\mathscr{\mathscr { E }}}^{\tilde{\mathscr{E}}}=H_{\mathscr{\mathscr { E }}}^{\tilde{E}} V_{v(1)} \tilde{\mathscr{E}} \oplus V_{v(2)} \tilde{\mathscr{E}} \oplus V_{c v(3)}^{*} \tilde{\mathscr{E}} \oplus \cdots \oplus V_{c v(z-1)}^{*} \tilde{\mathscr{E}} \oplus V_{v(z)} \tilde{\mathscr{E}} .
$$

Locally, an $\mathrm{N}$-connection $\tilde{\mathbf{N}}$ in $\tilde{\mathscr{E}}$ is given by its coefficients

$$
\begin{array}{cccccc}
N_{i}^{a_{1}}, & N_{i}^{a_{2}}, & N_{i a_{3}}, & \ldots, & N_{i a_{z-1}}, & N_{i}^{a_{z}}, \\
0, & N_{a_{1}}^{a_{2}}, & N_{a_{1} a_{3}}, & \ldots, & N_{a_{1} a_{z-1}}, & N_{a_{1}}^{a_{z}}, \\
0, & 0, & N_{a_{2} a_{3}}, & \ldots, & N_{a_{2} a_{z-1}}, & N_{a_{2}}^{a_{z}}, \\
\vdots & \vdots & \vdots & \vdots & \vdots & \vdots \\
0, & 0, & 0, & \ldots, & N_{a_{z-2} a_{z-1}}, & N_{a_{z-2}}^{a_{z}}, \\
0, & 0, & 0, & \ldots, & 0, & N^{a_{z-1} a_{z}},
\end{array}
$$

which are given with respect to the components of bases (2.14).

2.3.4. Anholonomic frames and N-connections. Having defined an N-connection structure in a (vector, covector, or higher-order vector/covector) bundle, we can adapt with respect to this structure (by "N-elongation") the operators of partial derivatives and differentials, and consider decompositions of geometrical objects with respect to adapted bases and cobases.

ANHOLONOMIC FRAMES IN V-BUNDLES. In a v-bundle $\mathscr{E}$ provided with an N-connection, we can adapt to this structure the geometric constructions by introducing locally adapted basis (N-frame or N-basis)

$$
\delta_{\alpha}=\frac{\delta}{\delta u^{\alpha}}=\left(\delta_{i}=\frac{\delta}{\delta x^{i}}=\partial_{i}-N_{i}^{a}(u) \partial_{a}, \partial_{a}=\frac{\partial}{\partial y^{a}}\right)
$$

and its dual N-basis (N-coframe or N-cobasis)

$$
\delta^{\alpha}=\delta u^{\alpha}=\left(d^{i}=\delta x^{i}=d x^{i}, \delta^{a}=\delta y^{a}+N_{i}^{a}(u) d x^{i}\right) .
$$


The anholonomic coefficients, $\mathbf{w}=\left\{w_{\beta \gamma}^{\alpha}(u)\right\}$, of N-frames are defined to satisfy the relations

$$
\left[\delta_{\alpha}, \delta_{\beta}\right]=\delta_{\alpha} \delta_{\beta}-\delta_{\beta} \delta_{\alpha}=w_{\beta \gamma}^{\alpha}(u) \delta_{\alpha}
$$

A frame basis is holonomic if all anholonomy coefficients vanish (like for usual coordinate basis (2.3)), or anholonomic if there are nonzero values of $w_{\beta \gamma}^{\alpha}$.

The operators (2.29) and (2.30) on a v-bundle $\mathscr{E}$ enabled with an N-connection can be considered as respective equivalents of the operators of partial derivations and differentials: the existence of an N-connection structure results in "elongation" of partial derivations on $x$-variables and in "elongation" of differentials on $y$-variables.

The algebra of tensorial distinguished fields $D T(\mathscr{E})$ (d-fields, d-tensors, d-objects) on $\mathscr{E}$ is introduced as the tensor algebra $\mathscr{T}=\left\{\mathscr{T}_{q s}^{p r}\right\}$ of the v-bundle $\mathscr{E}(d)=\left(H \mathscr{E} \oplus V_{\mathscr{E}}^{\mathscr{E}}, p_{d}, \mathscr{E}\right)$, where $p_{d}: H \mathscr{E} \oplus V \mathscr{E} \rightarrow \mathscr{E}$.

ANHOLONOMIC FRAMES IN CV-BUNDLES. The anholonomic frames adapted to the Ň-connection structure are introduced similarly to (2.29) and (2.30):

(i) the locally adapted basis ( $\mathrm{N}$-basis or $\check{N}$-frame):

$$
\breve{\delta}_{\alpha}=\frac{\breve{\delta}}{\delta u^{\alpha}}=\left(\delta_{i}=\frac{\delta}{\delta x^{i}}=\partial_{i}+\breve{N}_{i a}(\breve{u}) \breve{\partial}^{a}, \breve{\partial}^{a}=\frac{\partial}{\partial p_{a}}\right),
$$

(ii) its dual (Ň-cobasis or Ň-coframe):

$$
\breve{\delta}^{\alpha}=\breve{\delta} u^{\alpha}=\left(d^{i}=\delta x^{i}=d x^{i}, \breve{\delta}_{a}=\breve{\delta} p_{a}=d p_{a}-\breve{N}_{i a}(\breve{u}) d x^{i}\right) .
$$

We note that the sings of N-elongations are inverse to those for N-elongations.

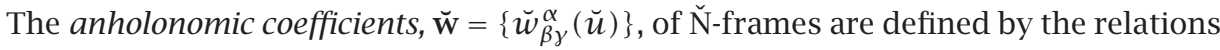

$$
\left[\breve{\delta}_{\alpha}, \breve{\delta}_{\beta}\right]=\breve{\delta}_{\alpha} \breve{\delta}_{\beta}-\breve{\delta}_{\beta} \breve{\delta}_{\alpha}=\breve{w}_{\beta \gamma}^{\alpha}(\breve{u}) \breve{\delta}_{\alpha} .
$$

The algebra of tensorial distinguished fields $D T(\breve{\mathscr{E}})$ (d-fields, d-tensors, d-objects) on $\breve{\mathscr{E}}$ is introduced as the tensor algebra $\breve{\mathscr{T}}=\left\{\breve{\mathscr{T}}_{q s}^{p r}\right\}$ of the cv-bundle $\breve{\mathscr{E}}(d)=\left(H{ }_{\mathscr{\mathscr { E }}} \oplus V^{\breve{\mathscr{E}}}, \breve{p}_{d}, \breve{\mathscr{E}}\right)$, where $\breve{p}_{d}: H_{\breve{\mathscr{E}}}^{\breve{ }} \oplus V^{\breve{\mathscr{E}}} \rightarrow \breve{\mathscr{E}}$.

An element $\breve{\mathbf{t}} \in \breve{\mathscr{T}}_{q \mathcal{q}}^{p r}$, d-tensor field of type $\left(\begin{array}{cc}p & r \\ q & s\end{array}\right)$, can be written in local form as

$$
\breve{\mathbf{t}}=\breve{t}_{j_{1} \cdots j_{q} b_{1} \cdots b_{r}}^{i_{1} \cdots i_{p} a_{1} \cdots a_{r}}(\breve{u}) \breve{\delta}_{i_{1}} \otimes \cdots \otimes \breve{\delta}_{i_{p}} \otimes \breve{\partial}_{a_{1}} \otimes \cdots \otimes \breve{\partial}_{a_{r}} \otimes \breve{d}^{j_{1}} \otimes \cdots \otimes \breve{d}^{j_{q}} \otimes \breve{\delta}^{b_{1}} \cdots \otimes \breve{\delta}^{b_{r}} .
$$

We will, respectively, use the denotations $\mathscr{L}(\breve{E})$ (or $\mathscr{L}(M)), \Lambda^{p}(\breve{\mathscr{E}})$ (or $\Lambda^{p}(M)$ ), and $\mathscr{F}(\breve{E})$ (or $\mathscr{F}(M)$ ) for the module of d-vector fields on $\breve{\mathscr{E}}$ (or $M$ ), the exterior algebra of p-forms on $\breve{\mathscr{E}}$ (or $M$ ), and the set of real functions on $\breve{\mathscr{E}}$ (or $M$ ).

ANHOLONOMIC FRAMES IN HVC-BUNDLES. The anholonomic frames adapted to an $\mathrm{N}$-connection in hvc-bundle $\tilde{\mathscr{E}}$ are defined by the set of coefficients (2.28); having restricted the constructions to a vector (or covector) shell, we obtain some generalizations of the formulas for the corresponding $\mathrm{N}$-(or Ň)-connection elongation of partial derivatives defined by (2.29) (or (2.32)) and (2.30) (or (2.33)). 
We introduce the adapted partial derivatives (anholonomic N-frames or N-bases) in $\tilde{\mathscr{E}}$ by applying the coefficients (2.28):

$$
\tilde{\delta}_{\alpha}=\frac{\tilde{\delta}}{\delta \tilde{u}^{\alpha}}=\left(\delta_{i}, \delta_{a_{1}}, \delta_{a_{2}}, \breve{\delta}^{a_{3}}, \ldots, \breve{\delta}^{a_{z-1}}, \partial_{a_{z}}\right),
$$

where

$$
\begin{aligned}
\delta_{i} & =\partial_{i}-N_{i}^{a_{1}} \partial_{a_{1}}-N_{i}^{a_{2}} \partial_{a_{2}}+N_{i a_{3}} \breve{\partial}^{a_{3}}-\cdots+N_{i a_{z-1}} \breve{\partial}^{a_{z-1}}-N_{i}^{a_{z}} \partial_{a_{z}}, \\
\delta_{a_{1}} & =\partial_{a_{1}}-N_{a_{1}}^{a_{2}} \partial_{a_{2}}+N_{a_{1} a_{3}} \breve{\partial}^{a_{3}}-\cdots+N_{a_{1} a_{z-1}} \breve{\partial}^{a_{z-1}}-N_{a_{1}}^{a_{z}} \partial_{a_{z}}, \\
\delta_{a_{2}} & =\partial_{a_{2}}+N_{a_{2} a_{3}} \breve{\partial}^{a_{3}}-\cdots+N_{a_{2} a_{z-1}} \breve{\partial}^{a_{z-1}}-N_{a_{2}}^{a_{z}} \partial_{a_{z}}, \\
\breve{\delta}^{a_{3}} & =\tilde{\partial}^{a_{3}}-N^{a_{3} a_{4}} \partial_{a_{4}}-\cdots+N_{a_{z-1}}^{a_{3}} \breve{\partial}^{a_{z-1}}-N^{a_{3} a_{z}} \partial_{a_{z}}, \\
& \vdots \\
\check{\delta}^{a_{z-1}} & =\tilde{\partial}^{a_{z-1}}-N^{a_{z-1} a_{z}} \partial_{a_{z}}, \\
\partial_{a_{z}} & =\frac{\partial}{\partial y^{a_{z}}} .
\end{aligned}
$$

These formulas can be written in the matrix form

$$
\tilde{\delta}_{\bullet}=\hat{\mathbf{N}}(u) \times \tilde{\partial}_{\bullet},
$$

where

$$
\begin{aligned}
& \tilde{\delta}_{\cdot}=\left(\begin{array}{c}
\delta_{i} \\
\delta_{a_{1}} \\
\delta_{a_{2}} \\
\breve{\delta}^{a_{3}} \\
\vdots \\
\breve{\delta}^{a_{z-1}} \\
\partial_{a_{z}}
\end{array}\right), \quad \tilde{\partial}_{\cdot}=\left(\begin{array}{c}
\partial_{i} \\
\partial_{a_{1}} \\
\partial_{a_{2}} \\
\tilde{\partial}^{a_{3}} \\
\vdots \\
\tilde{\partial}^{a_{z-1}} \\
\partial_{a_{z}}
\end{array}\right) \\
& \widehat{\mathbf{N}}=\left(\begin{array}{cccccccc}
1 & -N_{i}^{a_{1}} & -N_{i}^{a_{2}} & N_{i a_{3}} & -N_{i}^{a_{4}} & \cdots & N_{i a_{z-1}} & -N_{i}^{a_{z}} \\
0 & 1 & -N_{a_{1}}^{a_{2}} & N_{a_{1} a_{3}} & -N_{a_{1}}^{a_{4}} & \cdots & N_{a_{1} a_{z-1}} & -N_{a_{1}}^{a_{z}} \\
0 & 0 & 1 & N_{a_{2} a_{3}} & -N_{a_{2}}^{a_{4}} & \cdots & N_{a_{2} a_{z-1}} & -N_{a_{2}}^{a_{z}} \\
0 & 0 & 0 & 1 & -N^{a_{3} a_{4}} & \cdots & N_{a_{z-1}}^{a_{3}} & -N^{a_{3} a_{z}} \\
\vdots & \vdots & \vdots & \vdots & \vdots & \vdots & \vdots & \vdots \\
0 & 0 & 0 & 0 & 0 & \cdots & 1 & -N^{a_{z-1} a_{z}} \\
0 & 0 & 0 & 0 & 0 & \cdots & 0 & 1
\end{array}\right) .
\end{aligned}
$$

The adapted differentials (anholonomic N-coframes or N-cobases) in $\tilde{\mathscr{E}}$ are introduced in the simplest form by using the matrix formalism: the respective dual matrices

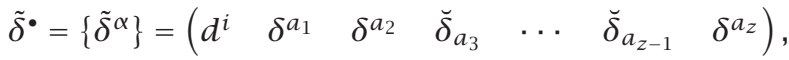

$$
\begin{aligned}
& \tilde{d}^{\bullet}=\left\{\tilde{\partial}^{\alpha}\right\}=\left(\begin{array}{lllllll}
d^{i} & d^{a_{1}} & d^{a_{2}} & d_{a_{3}} & \cdots & d_{a_{z-1}} & d^{a_{z}}
\end{array}\right)
\end{aligned}
$$


are related via a matrix relation

$$
\tilde{\delta}^{\bullet}=\tilde{d} \cdot \hat{\mathbf{M}}
$$

which defines the formulas for anholonomic N-coframes. The matrix $\widehat{\mathbf{M}}$ from (2.41) is the inverse to $\hat{\mathbf{N}}$, that is, it satisfies the condition

$$
\widehat{\mathbf{M}} \times \hat{\mathbf{N}}=I .
$$

The anholonomic coefficients, $\widetilde{\mathbf{w}}=\left\{\widetilde{w}_{\beta \gamma}^{\alpha}(\tilde{u})\right\}$, on hcv-bundle $\tilde{\mathscr{E}}$ are expressed via coefficients of the matrix $\hat{\mathbf{N}}$ and their partial derivatives following the relations

$$
\left[\tilde{\delta}_{\alpha}, \tilde{\delta}_{\beta}\right]=\tilde{\delta}_{\alpha} \tilde{\delta}_{\beta}-\tilde{\delta}_{\beta} \tilde{\delta}_{\alpha}=\widetilde{w}_{\beta \gamma}^{\alpha}(\tilde{u}) \tilde{\delta}_{\alpha}
$$

We omit the explicit formulas on shells.

A d-tensor formalism can also be developed on the space $\tilde{\mathscr{E}}$. In this case the indices have to be stipulated for every shell separately, like for v-bundles or cv-bundles.

3. Distinguished connections and metrics. In general, distinguished objects (dobjects) on a v-bundle $\mathscr{E}$ (or cv-bundle $\breve{\mathscr{E}}$ ) are introduced as geometric objects with various group and coordinate transforms coordinated with the N-connection structure on $\mathscr{E}$ (or $\breve{\mathscr{E}}$ ). For example, a distinguished connection (in brief, d-connection) $D$ on $\mathscr{E}$ (or $\breve{\mathscr{C}}$ ) is defined as a linear connection $D$ on $E$ (or $\breve{E}$ ) conserving under a parallelism the global decomposition (2.22) (or (2.23)) into horizontal and vertical subbundles of $T_{\mathscr{E}}^{\mathscr{E}}$ (or $T \breve{\mathscr{E}}$ ). A covariant derivation associated to a d-connection becomes d-covariant. We will give necessary formulas for cv-bundles in round brackets.

\section{1. d-connections}

3.1.1. d-connections in v-bundles (cv-bundles). An N-connection in a v-bundle $\mathscr{E}$ (cv-bundle $\breve{\mathscr{C}}$ ) induces a corresponding decomposition of d-tensors into sums of horizontal and vertical parts, for example, for every d-vector $X \in \mathscr{X}(\mathscr{E})(\breve{X} \in \mathscr{L}(\breve{\mathscr{C}}))$ and 1-form $A \in \Lambda^{1}(\mathscr{E})\left(\breve{A} \in \Lambda^{1}(\breve{\mathscr{E}})\right)$, we have respectively

$$
\begin{gathered}
X=h X+v X, \quad A=h A+v A, \\
(\breve{X}=h \breve{X}+v X, \breve{A}=h \breve{A}+v \breve{A}),
\end{gathered}
$$

where

$$
\begin{gathered}
h X=X^{i} \delta_{i}, \quad v X=X^{a} \partial_{a}, \\
\left(h \breve{X}=\breve{X}^{i} \tilde{\delta}_{i}, v \breve{X}=\breve{X}_{a} \breve{\partial}^{a}\right), \\
h A=A_{i} \delta^{i}, \quad v A=A_{a} d^{a}, \\
\left(h \breve{A}=\breve{A}_{i} \breve{\delta}^{i}, v \breve{A}=\breve{A}^{a} \breve{d}_{a}\right) .
\end{gathered}
$$


In consequence, we can associate to every d-covariant derivation along the d-vector (3.1), $D_{X}=X \circ D\left(D_{\breve{X}}=\breve{X} \circ D\right)$, two new operators of h- and v-covariant derivations

$$
\begin{gathered}
D_{X}^{(h)} Y=D_{h X} Y, \quad D_{X}^{(v)} Y=D_{v X} Y, \quad \forall Y \in \mathscr{X}(\mathscr{E}), \\
\left(D_{\breve{X}}^{(h)} \breve{Y}=D_{h \breve{X}} \breve{Y}, D_{\breve{X}}^{(v)} \breve{Y}=D_{v \breve{X}} \breve{Y}, \forall \breve{Y} \in \mathscr{X}(\breve{\mathscr{E}})\right)
\end{gathered}
$$

for which the following conditions hold:

$$
\begin{aligned}
D_{X} Y & =D_{X}^{(h)} Y+D_{X}^{(v)} Y, \\
\left(D_{\breve{X}} \breve{Y}\right. & \left.=D_{\breve{X}}^{(h)} \breve{Y}+D_{\breve{X}}^{(v)} \breve{Y}\right),
\end{aligned}
$$

where

$$
\begin{aligned}
& D_{X}^{(h)} f=(h X) f, \quad D_{X}^{(v)} f=(v X) f, \quad X, Y \in \mathscr{X}(\mathscr{E}), f \in \mathscr{F}(M), \\
& \left(\breve{D}_{\breve{X}}^{(h)} f=(h \breve{X}) f, \breve{D}_{\breve{X}}^{(v)} f=(v \breve{X}) f, \breve{X}, \breve{Y} \in \mathscr{X}(\breve{\mathscr{E}}), f \in \mathscr{F}(M)\right) .
\end{aligned}
$$

The components $\Gamma_{\beta \gamma}^{\alpha}\left(\breve{\Gamma}_{\beta \gamma}^{\alpha}\right)$ of a d-connection $\breve{D}_{\alpha}=\left(\breve{\delta}_{\alpha} \circ D\right)$, locally adapted to the Nconnection structure with respect to the frames (2.29) and (2.30) ((2.32) and (2.33)), are defined by the equations

$$
D_{\alpha} \delta_{\beta}=\Gamma_{\alpha \beta}^{\gamma} \delta_{\gamma}, \quad\left(\breve{D}_{\alpha} \breve{\delta}_{\beta}=\breve{\Gamma}_{\alpha \beta}^{\gamma} \breve{\delta}_{\gamma}\right),
$$

from which one immediately has

$$
\Gamma_{\alpha \beta}^{\gamma}(u)=\left(D_{\alpha} \delta_{\beta}\right) \circ \delta^{\gamma}, \quad\left(\breve{\Gamma}_{\alpha \beta}^{\gamma}(\breve{u})=\left(\breve{D}_{\alpha} \breve{\delta}_{\beta}\right) \circ \breve{\delta}^{\gamma}\right)
$$

The coefficients of operators of $h$ - and v-covariant derivations

$$
\begin{aligned}
& D_{k}^{(h)}=\left\{L_{j k}^{i}, L_{b k}^{a}\right\}, \quad D_{c}^{(v)}=\left\{C_{j k}^{i}, C_{b c}^{a}\right\}, \\
& \left(\breve{D}_{k}^{(h)}=\left\{\breve{L}_{j k}^{i}, \breve{L}_{a k}^{b}\right\}, \breve{D}^{(v) c}=\left\{\breve{C}_{j}^{i c}, \breve{C}_{a}^{b c}\right\}\right)
\end{aligned}
$$

(see (3.4)) are introduced as corresponding h- and v-parametrizations of (3.7)

$$
\begin{aligned}
& L_{j k}^{i}=\left(D_{k} \delta_{j}\right) \circ d^{i}, \quad L_{b k}^{a}=\left(D_{k} \partial_{b}\right) \circ \delta^{a}, \\
& \left(\breve{L}_{j k}^{i}=\left(\breve{D}_{k} \breve{\delta}_{j}\right) \circ d^{i}, \breve{L}_{a k}^{b}=\left(\breve{D}_{k} \breve{\partial}^{b}\right) \circ \breve{\delta}_{a}\right), \\
& C_{j c}^{i}=\left(D_{c} \delta_{j}\right) \circ d^{i}, \quad C_{b c}^{a}=\left(D_{c} \partial_{b}\right) \circ \delta^{a}, \\
& \left(\breve{C}_{j}^{i c}=\left(\breve{D}^{c} \breve{\delta}_{j}\right) \circ d^{i}, \breve{C}_{a}^{b c}=\left(\breve{D}^{c} \breve{\partial}^{b}\right) \circ \breve{\delta}_{a}\right) \text {. }
\end{aligned}
$$

A set of components (3.9) and (3.10)

$$
\Gamma_{\alpha \beta}^{\gamma}=\left[L_{j k}^{i}, L_{b k}^{a}, C_{j c}^{i}, C_{b c}^{a}\right], \quad\left(\breve{\Gamma}_{\alpha \beta}^{\gamma}=\left[\breve{L}_{j k}^{i}, \breve{L}_{a k}^{b}, \breve{C}_{j}^{i c}, \breve{C}_{a}^{b c}\right]\right)
$$

completely defines the local action of a d-connection $D$ in $\mathscr{E}(\breve{D}$ in $\breve{\mathscr{E}})$.

For instance, having taken on $\mathscr{E}(\breve{\mathscr{C}})$ a d-tensor field of type $\left(\begin{array}{ll}1 & 1 \\ 1 & 1\end{array}\right)$,

$$
\mathbf{t}=t_{j b}^{i a} \delta_{i} \otimes \partial_{a} \otimes d^{j} \otimes \delta^{b}, \quad \tilde{\mathbf{t}}=\breve{t}_{j a}^{i b} \breve{\delta}_{i} \otimes \breve{\partial}^{a} \otimes d^{j} \otimes \breve{\delta}_{b},
$$


and a d-vector $\mathbf{X}(\breve{\mathbf{X}})$, we obtain

$$
\begin{aligned}
D_{X} \mathbf{t} & =D_{X}^{(h)} \mathbf{t}+D_{X}^{(v)} \mathbf{t}=\left(X^{k} \breve{t}_{j b \mid k}^{i a}+X^{c} t_{j b \perp c}^{i a}\right) \delta_{i} \otimes \partial_{a} \otimes d^{j} \otimes \delta^{b}, \\
\left(\breve{D}_{\breve{X}} \tilde{\mathbf{t}}\right. & \left.=\breve{D}_{\breve{X}}^{(h)} \tilde{\mathbf{t}}+\breve{D}_{\breve{X}}^{(v)} \tilde{\mathbf{t}}=\left(\breve{X}^{k} \breve{t}_{j a \mid k}^{i b}+\breve{X}_{c} \breve{t}_{j a}^{i b \perp c}\right) \breve{\delta}_{i} \otimes \breve{\partial}^{a} \otimes d^{j} \otimes \breve{\delta}_{b}\right),
\end{aligned}
$$

where the h-covariant derivative is written as

$$
\begin{aligned}
& t_{j b \mid k}^{i a}=\delta_{k} t_{j b}^{i a}+L_{h k}^{i} t_{j b}^{h a}+L_{c k}^{a} t_{j b}^{i c}-L_{j k}^{h} t_{h b}^{i a}-L_{b k}^{c} t_{j c}^{i a}, \\
& \left(\breve{t}_{j a \mid k}^{i b}=\breve{\delta}_{k} \breve{t}_{j a}^{i b}+\breve{L}_{h k}^{i} \breve{t}_{j a}^{h b}+\breve{L}_{c k}^{b} \breve{t}_{j a}^{i c}-\breve{L}_{j k}^{h} \breve{t}_{h a}^{i b}-\breve{L}_{c k}^{b} \breve{t}_{j a}^{i c}\right)
\end{aligned}
$$

and the $\mathrm{v}$-covariant derivative is written as

$$
\begin{aligned}
t_{j b \perp c}^{i a} & =\partial_{c} t_{j b}^{i a}+C_{h c}^{i} t_{j b}^{h a}+C_{d c}^{a} t_{j b}^{i d}-C_{j c}^{h} t_{h b}^{i a}-C_{b c}^{d} t_{j d}^{i a}, \\
\left(\breve{t}_{j a}^{i b \perp c}\right. & \left.=\breve{\partial}^{c} \breve{t}_{j a}^{i b}+\breve{C}_{j}^{i c} \breve{t}_{j a}^{h b}+\breve{C}_{a}^{d c} \breve{t}_{j d}^{i b}-\breve{C}_{j}^{i c} \breve{t}_{h a}^{i b}-\breve{C}_{d}^{b c} \breve{t}_{j a}^{i d}\right) .
\end{aligned}
$$

For a scalar function $f \in \mathscr{F}(\mathscr{E})\left(f \in \mathscr{F}_{(}(\breve{\mathscr{C}})\right)$, we have

$$
\begin{aligned}
& D_{k}^{(h)}=\frac{\delta f}{\delta x^{k}}=\frac{\partial f}{\partial x^{k}}-N_{k}^{a} \frac{\partial f}{\partial y^{a}}, \quad D_{c}^{(v)} f=\frac{\partial f}{\partial y^{c}}, \\
& \left(\breve{D}_{k}^{(h)}=\frac{\breve{\delta} f}{\delta x^{k}}=\frac{\partial f}{\partial x^{k}}+N_{k a} \frac{\partial f}{\partial p_{a}}, \breve{D}^{(v) c} f=\frac{\partial f}{\partial p_{c}}\right) .
\end{aligned}
$$

3.1.2. d-connections in hvc-bundles. The theory of connections in higher-order anisotropic vector superbundles and vector bundles was elaborated in [90, 91, 92, 93]. Here, we reformulate that formalism for the case when some shells of higher-order anisotropy could be covector spaces by stating the general rules of covariant derivation compatible with the N-connection structure in hvc-bundle $\tilde{\mathscr{E}}$, and omit details and cumbersome formulas.

For an hvc-bundle of type $\tilde{\mathscr{E}}=\tilde{\mathscr{E}}[v(1), v(2), c v(3), \ldots, c v(z-1), v(z)]$, a d-connection $\tilde{\Gamma}_{\alpha \beta}^{\gamma}$ has the next shell decomposition of components (with induction being on the $p$ th shell, considered as the base space, which is in this case an hvc-bundle, we introduce in a usual manner, like a vector or covector fiber, the $(p+1)$ th shell):

$$
\begin{aligned}
\tilde{\Gamma}_{\alpha \beta}^{\gamma}=\left\{\Gamma_{\alpha_{1} \beta_{1}}^{\gamma_{1}}=\left[L_{j_{1} k_{1}}^{i_{1}}, L_{b_{1} k_{1}}^{a_{1}}, C_{j_{1} c_{1}}^{i_{1}}, C_{b_{1} c_{1}}^{a_{1}}\right],\right. \\
\Gamma_{\alpha_{2} \beta_{2}}^{\gamma_{2}}=\left[L_{j_{2} k_{2}}^{i_{2}}, L_{b_{2} k_{2}}^{a_{2}}, C_{j_{2} c_{2}}^{i_{2}}, C_{b_{2} c_{2}}^{a_{2}}\right], \\
\breve{\Gamma}_{\alpha_{3} \beta_{3}}^{\gamma_{3}}=\left[\breve{L}_{j_{3} k_{3}}^{i_{3}}, \breve{L}_{a_{3} k_{3}}^{b_{3}}, \breve{C}_{j_{3}}^{i_{3} c_{3}}, \breve{C}_{a_{3}}^{b_{3} c_{3}}\right], \ldots, \\
\breve{\Gamma}_{\alpha_{z-1} \beta_{z-1}}^{\gamma_{z-1}}=\left[\breve{L}_{j_{z-1} k_{z-1}}^{i_{z-1}}, \breve{L}_{a_{z-1} k_{z-1}}^{b_{z-1}}, \breve{C}_{j_{z-1}}^{i_{z-1} c_{z-1}}, \breve{C}_{a_{z-1}}^{b_{z-1} c_{z-1}}\right], \\
\left.\Gamma_{\alpha_{z} \beta_{z}}^{\gamma_{z}}=\left[L_{j_{z} k_{z}}^{i_{z}}, L_{b_{z} k_{z}}^{a_{z}}, C_{j_{z} c_{z}}^{i_{z}}, C_{b_{z} c_{z}}^{a_{z}}\right]\right\} .
\end{aligned}
$$

These coefficients determine the rules of a covariant derivation $\tilde{D}$ on $\tilde{\mathscr{E}}$. 


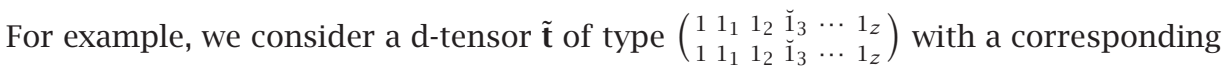
tensor product of components of anholonomic N-frames (2.38) and (2.41):

$$
\begin{aligned}
\tilde{\mathbf{t}}=\tilde{t}_{j b_{1} b_{2} \breve{a}_{3} \cdots \breve{a}_{z-1} a_{z} a_{2} \breve{b}_{z} \breve{b}_{z-1} a_{z}} & \otimes \partial_{a_{1}} \otimes d^{j} \otimes \delta^{b_{1}} \otimes \partial_{a_{2}} \otimes \delta^{b_{2}} \otimes \breve{\partial}^{a_{3}} \\
& \otimes \breve{\delta}_{b_{3}} \cdots \otimes \breve{\partial}^{a_{z-1}} \otimes \breve{\delta}_{b z-1} \otimes \partial_{a_{z}} \otimes \delta^{b_{z}}
\end{aligned}
$$

The d-covariant derivation $\tilde{D}$ of $\tilde{\mathbf{t}}$ is to be performed separately for every shell according to the rule (3.15) if a shell is defined by a vector subspace, or according to the rule (3.16) if the shell is defined by a covector subspace.

\subsection{Metric structure}

3.2.1. d-metrics in $\mathbf{v}$-bundles. We define a metric structure $\mathbf{G}$ in the total space $E$ of a v-bundle $\mathscr{E}=(E, p, M)$ over a connected and paracompact base $M$ as a symmetric covariant tensor field of type $(0,2)$,

$$
\mathbf{G}=G_{\alpha \beta} d u^{\alpha} \otimes d u^{\beta},
$$

being nondegenerate and of constant signature on $E$.

Nonlinear connection $\mathbf{N}$ and metric $\mathbf{G}$ structures on $\mathscr{E}$ are mutually compatible if they satisfy the following conditions:

$$
\mathbf{G}\left(\delta_{i}, \partial_{a}\right)=0
$$

or equivalently,

$$
G_{i a}(u)-N_{i}^{b}(u) h_{a b}(u)=0,
$$

where $h_{a b}=\mathbf{G}\left(\partial_{a}, \partial_{b}\right)$ and $G_{i a}=\mathbf{G}\left(\partial_{i}, \partial_{a}\right)$, which gives

$$
N_{i}^{b}(u)=h^{a b}(u) G_{i a}(u)
$$

(the matrix $h^{a b}$ is inverse to $h_{a b}$ ). One obtains the following decomposition of metric:

$$
\mathbf{G}(X, Y)=\mathbf{h G}(X, Y)+\mathbf{v G}(X, Y),
$$

where the d-tensor $\mathbf{h G}(X, Y)=\mathbf{G}(h X, h Y)$ is of type $\left(\begin{array}{ll}0 & 0 \\ 2 & 0\end{array}\right)$ and the d-tensor $\mathbf{v G}(X, Y)=$ $\mathbf{G}(v X, v Y)$ is of type $\left(\begin{array}{ll}0 & 0 \\ 0 & 2\end{array}\right)$. With respect to the anholonomic basis (2.29), the d-metric (3.24) is written as

$$
\mathbf{G}=g_{\alpha \beta}(u) \delta^{\alpha} \otimes \delta^{\beta}=g_{i j}(u) d^{i} \otimes d^{j}+h_{a b}(u) \delta^{a} \otimes \delta^{b},
$$

where $g_{i j}=\mathbf{G}\left(\delta_{i}, \delta_{j}\right)$.

A metric structure of type (3.24) (equivalently, of type (3.25)) or a metric on $E$ with components satisfying the constraints (3.21) and (3.22) (equivalently (3.23)) defines an adapted to the given $\mathrm{N}$-connection inner (d-scalar) product on the tangent bundle $\mathscr{T} \mathscr{E}$. 
A d-connection $D_{X}$ is metric (or compatible with metric $\mathbf{G}$ ) on $\mathscr{E}$ if $D_{X} \mathbf{G}=0$, for all $X \in \mathscr{X}(\mathscr{E})$. With respect to anholonomic frames, these conditions are written as

$$
D_{\alpha} \mathcal{G}_{\beta \gamma}=0
$$

where by $g_{\beta \gamma}$ we denote the coefficients in the block form (3.25).

3.2.2. d-metrics in cv- and hvc-bundles. The presented considerations on the selfconsistent definition of N-connection, d-connection, and metric structures in v-bundles can be reformulated in a similar fashion for other types of anisotropic space-times, on cv-bundles and on shells of hvc-bundles. For simplicity, we give here only the analogous formulas for the metric d-tensor (3.25).

(i) On cv-bundle $\breve{\mathscr{E}}$ we write

$$
\breve{\mathbf{G}}=\breve{g}_{\alpha \beta}(\breve{u}) \breve{\delta}^{\alpha} \otimes \breve{\delta}^{\beta}=\breve{g}_{i j}(\breve{u}) d^{i} \otimes d^{j}+\breve{h}^{a b}(\breve{u}) \breve{\delta}_{a} \otimes \breve{\delta}_{b},
$$

where $\breve{g}_{i j}=\breve{\mathbf{G}}\left(\breve{\delta}_{i}, \breve{\delta}_{j}\right), \breve{h}^{a b}=\breve{\mathbf{G}}\left(\breve{\partial}^{a}, \breve{\partial}^{b}\right)$, and the N-coframes are given by formulas (2.33).

For simplicity, we consider that the metricity conditions are satisfied, $\breve{D}_{\gamma} \breve{g}_{\alpha \beta}$ $=0$.

(ii) On hvc-bundle $\tilde{\mathscr{E}}$ we write

$$
\begin{aligned}
\tilde{\mathbf{G}}= & \tilde{g}_{\alpha \beta}(\tilde{u}) \tilde{\delta}^{\alpha} \otimes \tilde{\delta}^{\beta} \\
= & \tilde{g}_{i j}(\tilde{u}) d^{i} \otimes d^{j}+\tilde{h}_{a_{1} b_{1}}(\tilde{u}) \delta^{a_{1}} \otimes \delta^{b_{1}}+\tilde{h}_{a_{2} b_{2}}(\tilde{u}) \delta^{a_{2}} \otimes \delta^{b_{2}} \\
& +\tilde{h}^{a_{3} b_{3}}(\tilde{u}) \breve{\delta}_{a_{3}} \otimes \breve{\delta}_{b_{3}}+\cdots+\tilde{h}^{a_{z-1} b_{z-1}}(\tilde{u}) \breve{\delta}_{a_{z-1}} \otimes \breve{\delta}_{b_{z-1}}+\tilde{h}_{a_{z} b_{z}}(\tilde{u}) \delta^{a_{z}} \otimes \delta^{b_{z}},
\end{aligned}
$$

where $\tilde{g}_{i j}=\tilde{\mathbf{G}}\left(\tilde{\delta}_{i}, \tilde{\delta}_{j}\right), \tilde{h}_{a_{1} b_{1}}=\tilde{\mathbf{G}}\left(\partial_{a_{1}}, \partial_{b_{1}}\right), \tilde{h}_{a_{2} b_{2}}=\tilde{\mathbf{G}}\left(\partial_{a_{2}}, \partial_{b_{2}}\right), \tilde{h}^{a_{3} b_{3}}=\tilde{\mathbf{G}}\left(\tilde{\partial}^{a_{3}}\right.$, $\left.\breve{\partial}^{b_{3}}\right), \ldots$, and the N-coframes are given by formula (2.41).

The metricity conditions are $\tilde{D}_{\gamma} \tilde{\mathfrak{g}}_{\alpha \beta}=0$.

(iii) On osculator bundle $T^{2} M=\mathrm{Osc}^{2} M$, we have a particular case of (3.28) when

$$
\tilde{\mathbf{G}}=\tilde{g}_{\alpha \beta}(\tilde{u}) \tilde{\delta}^{\alpha} \otimes \tilde{\delta}^{\beta}=\tilde{g}_{i j}(\tilde{u}) d^{i} \otimes d^{j}+\tilde{h}_{i j}(\tilde{u}) \delta y_{(1)}^{i} \otimes \delta y_{(1)}^{i}+\tilde{h}_{i j}(\tilde{u}) \delta y_{(2)}^{i} \otimes \delta y_{(2)}^{i}
$$

with respect to $\mathrm{N}$-coframes.

(iv) On dual osculator bundle $\left(T^{* 2} M, p^{* 2}, M\right)$ we have another particular case of (3.28) when

$$
\tilde{\mathbf{G}}=\tilde{g}_{\alpha \beta}(\tilde{u}) \tilde{\delta}^{\alpha} \otimes \tilde{\delta}^{\beta}=\tilde{g}_{i j}(\tilde{u}) d^{i} \otimes d^{j}+\tilde{h}_{i j}(\tilde{u}) \delta y_{(1)}^{i} \otimes \delta y_{(1)}^{i}+\tilde{h}^{i j}(\tilde{u}) \delta p_{i}^{(2)} \otimes \delta p_{i}^{(2)}
$$

with respect to N-coframes.

3.3. Some examples of d-connections. We emphasize that the geometry of connections in a v-bundle $\mathscr{E}$ is very rich. If a triple of fundamental geometric objects $\left(N_{i}^{a}(u), \Gamma_{\beta \gamma}^{\alpha}(u), g_{\alpha \beta}(u)\right)$ is fixed on $\mathscr{E}$, a multiconnection structure (with corresponding different rules of covariant derivation, which are, or not, mutually compatible and 
with the same, or without, induced d-scalar products in $\mathscr{T} \mathscr{E})$ is defined on this v-bundle. We can give a priority to a connection structure following some physical arguments, like the reduction to the Christoffel symbols in the holonomic case, mutual compatibility between metric and N-connection, and d-connection structures, and so on.

In this subsection, we enumerate some of the connections and covariant derivations in v-bundle $\mathscr{E}$, cv-bundle $\breve{\mathscr{E}}$, and in some hvc-bundles which can represent interest in the investigation of locally anisotropic gravitational and matter field interactions.

(1) Every N-connection in $\mathscr{E}$ with coefficients $N_{i}^{a}(x, y)$ being differentiable on $y$ variables induces a structure of linear connection $N_{\beta \gamma}^{\alpha}$, where

$$
N_{b i}^{a}=\frac{\partial N_{i}^{a}}{\partial y^{b}}, \quad N_{b c}^{a}(x, y)=0
$$

For some $Y(u)=Y^{i}(u) \partial_{i}+Y^{a}(u) \partial_{a}$ and $B(u)=B^{a}(u) \partial_{a}$, one introduces a covariant derivation as

$$
D_{Y}^{(\tilde{N})} B=\left[Y^{i}\left(\frac{\partial B^{a}}{\partial x^{i}}+N_{b i}^{a} B^{b}\right)+Y^{b} \frac{\partial B^{a}}{\partial y^{b}}\right] \frac{\partial}{\partial y^{a}}
$$

(2) The d-connection of Berwald type on v-bundle $\mathscr{E}$ (cv-bundle $\breve{\mathscr{E}}$ )

$$
\begin{gathered}
\Gamma_{\beta \gamma}^{(B) \alpha}=\left(L_{j k}^{i}, \frac{\partial N_{k}^{a}}{\partial y^{b}}, 0, C_{b c}^{a}\right), \\
\left(\breve{\Gamma}_{\beta \gamma}^{(B) \alpha}=\left(\breve{L}_{j k}^{i},-\frac{\partial \breve{N}_{k a}}{\partial p_{b}}, 0, \breve{C}_{a}^{b c}\right)\right),
\end{gathered}
$$

where

$$
\begin{gathered}
L_{. j k}^{i}(x, y)=\frac{1}{2} g^{i r}\left(\frac{\delta g_{j k}}{\delta x^{k}}+\frac{\delta g_{k r}}{\delta x^{j}}-\frac{\delta g_{j k}}{\delta x^{r}}\right), \\
C_{. b c}^{a}(x, y)=\frac{1}{2} h^{a d}\left(\frac{\partial h_{b d}}{\partial y^{c}}+\frac{\partial h_{c d}}{\partial y^{b}}-\frac{\partial h_{b c}}{\partial y^{d}}\right), \\
\left(\breve{L}_{. j k}^{i}(x, p)=\frac{1}{2} \breve{g}^{i r}\left(\frac{\breve{\delta} \breve{g}_{j k}}{\delta x^{k}}+\frac{\check{\delta} \breve{g}_{k r}}{\delta x^{j}}-\frac{\check{\delta} \breve{g}_{j k}}{\delta x^{r}}\right),\right. \\
\left.\breve{C}_{a}^{b c}(x, p)=\frac{1}{2} \breve{h}_{a d}\left(\frac{\partial \breve{h}^{b d}}{\partial p_{c}}+\frac{\partial \breve{h}^{c d}}{\partial p_{b}}-\frac{\partial \breve{h}^{b c}}{\partial p_{d}}\right)\right),
\end{gathered}
$$

which is hv-metric, that is, the conditions $D_{k}^{(B)} g_{i j}=0$ and $D_{c}^{(B)} h_{a b}=0\left(\breve{D}_{k}^{(B)} \breve{g}_{i j}=0\right.$ and $\breve{D}^{(B) c} \breve{h}^{a b}=0$ ) are satisfied.

(3) The canonical d-connection $\Gamma^{(\mathbf{c})}$ (or $\breve{\Gamma}^{(\mathbf{c})}$ ) on a v-bundle (or cv-bundle) is associated to a metric $\mathbf{G}$ (or $\breve{\mathbf{G}}$ ) of type (3.25) (or (3.27)),

$$
\begin{aligned}
\Gamma_{\beta \gamma}^{(c) \alpha} & =\left[L_{j k}^{(c) i}, L_{b k}^{(c) a}, C_{j c}^{(c) i}, C_{b c}^{(c) a}\right], \\
\left(\breve{\Gamma}_{\beta \gamma}^{(c) \alpha}\right. & \left.=\left[\breve{L}_{j k}^{(c) i}, \breve{L}_{a \cdot k}^{(c) . b}, \breve{C}_{j}^{(c) i c}, \breve{C}_{a}^{(c) b c}\right]\right)
\end{aligned}
$$


with coefficients

$$
\begin{gathered}
L_{j k}^{(c) i}=L_{. j k}^{i}, \quad C_{b c}^{(c) a}=C_{. b c}^{a}, \quad\left(\breve{L}_{j k}^{(c) i}=\breve{L}_{. j k}^{i}, \breve{C}_{a}^{(c) b c}=\breve{C}_{a}^{b c}\right), \quad(\text { see }(3.34)) \\
L_{b i}^{(c) a}=\frac{\partial N_{i}^{a}}{\partial y^{b}}+\frac{1}{2} h^{a c}\left(\frac{\delta h_{b c}}{\delta x^{i}}-\frac{\partial N_{i}^{d}}{\partial y^{b}} h_{d c}-\frac{\partial N_{i}^{d}}{\partial y^{c}} h_{d b}\right) \\
\left(\breve{L}_{a . i}^{(c) . b}=-\frac{\partial \breve{N}_{i}^{a}}{\partial p_{b}}+\frac{1}{2} \breve{h}_{a c}\left(\frac{\breve{\delta} \breve{h}^{b c}}{\delta x^{i}}+\frac{\partial \breve{N}_{i d}}{\partial p_{b}} \breve{h}^{d c}+\frac{\partial \breve{N}_{i d}}{\partial p_{c}} \breve{h}^{d b}\right)\right) \\
C_{j c}^{(c) i}=\frac{1}{2} g^{i k} \frac{\partial g_{j k}}{\partial y^{c}}, \quad\left(\breve{C}_{j}^{(c) i c}=\frac{1}{2} \breve{g}^{i k} \frac{\partial \breve{g}_{j k}}{\partial p_{c}}\right) .
\end{gathered}
$$

This is a metric d-connection which satisfies the conditions

$$
\begin{gathered}
D_{k}^{(c)} g_{i j}=0, \quad D_{c}^{(c)} g_{i j}=0, \quad D_{k}^{(c)} h_{a b}=0, \quad D_{c}^{(c)} h_{a b}=0, \\
\left(\breve{D}_{k}^{(c)} \breve{g}_{j k}=0, \breve{D}^{(c) c} \breve{g}_{j k}=0, \breve{D}_{k}^{(c)} \breve{h}^{b c}=0, \breve{D}^{(c) c} \breve{h}^{a b}=0\right) .
\end{gathered}
$$

In physical applications, we will use the canonical connection and, for simplicity, we will omit the index $(c)$. The coefficients (3.36) are to be extended to a higher order if we are dealing with derivations of geometrical objects with "shell" indices. In this case the fiber indices are to be stipulated for every type of shell in consideration.

(4) We can consider the N-adapted Christoffel symbols

$$
\tilde{\Gamma}_{\beta \gamma}^{\alpha}=\frac{1}{2} g^{\alpha \tau}\left(\delta_{\gamma} g_{\tau \beta}+\delta_{\beta} g_{\tau \gamma}-\delta g_{\beta \gamma}\right)
$$

which have the components of d-connection $\widetilde{\Gamma}_{\beta \gamma}^{\alpha}=\left(L_{j k}^{i}, 0,0, C_{b c}^{a}\right)$, with $L_{j k}^{i}$ and $C_{b c}^{a}$ as in (3.34) if $g_{\alpha \beta}$ is taken in the form (3.25).

3.4. Almost Hermitian anisotropic spaces. There are possible very interesting particular constructions $[45,46,47,48,49,50,51,52]$ on t-bundle $T M$ provided with $\mathrm{N}$-connection which defines an $\mathrm{N}$-adapted frame structure $\delta_{\alpha}=\left(\delta_{i}, \dot{\partial}_{i}\right)$ (for the same formulas (2.29) and (2.30) but with identified fiber and base indices). We use the dotted symbol in order to distinguish the horizontal and vertical operators because on t-bundles the indices could take the same values both for the base and fiber objects. This allows us to define an almost complex structure $\mathbf{J}=\left\{J_{\alpha}^{\beta}\right\}$ on $T M$ as follows:

$$
\mathbf{J}\left(\delta_{i}\right)=-\dot{\partial}_{i}, \quad \mathbf{J}\left(\dot{\partial}_{i}\right)=\delta_{i}
$$

It is obvious that $\mathbf{J}$ is well defined and $\mathrm{J}^{2}=-I$.

For d-metrics of type (3.25), on $T M$, we can consider the case when $g_{i j}(x, y)=$ $h_{a b}(x, y)$, that is,

$$
\mathbf{G}_{(t)}=g_{i j}(x, y) d x^{i} \otimes d x^{j}+g_{i j}(x, y) \delta y^{i} \otimes \delta y^{j},
$$

where the index $(t)$ denotes that we have a geometrical object defined on a tangent space. 
An almost complex structure $J_{\alpha}^{\beta}$ is compatible with a d-metric of type (3.40) and a d-connection $D$ on tangent bundle $T M$ if the conditions

$$
J_{\alpha}^{\beta} J_{\gamma}^{\delta} g_{\beta \delta}=g_{\alpha \gamma}, \quad D_{\alpha} J_{\beta}^{\gamma}=0
$$

are satisfied.

The pair $\left(\mathbf{G}_{(t)}, \mathbf{J}\right)$ is an almost Hermitian structure on $T M$.

One can introduce an almost sympletic 2-form associated to the almost Hermitian structure $\left(\mathbf{G}_{(t)}, \mathbf{J}\right)$,

$$
\theta=g_{i j}(x, y) \delta y^{i} \wedge d x^{j} .
$$

If the 2-form (3.42), defined by the coefficients $g_{i j}$, is closed, we obtain an almost Kähler structure in $T M$.

DEFINITION 3.1. An almost Kähler metric connection is a linear connection $D^{(H)}$ on $T \tilde{M}=T M \backslash\{0\}$ with the following properties:

(1) $D^{(H)}$ preserves by parallelism the vertical distribution defined by the N-connection structure;

(2) $D^{(H)}$ is compatible with the almost Kähler structure $\left(\mathbf{G}_{(t)}, \mathbf{J}\right)$, that is,

$$
D_{X}^{(H)} \mathfrak{g}=0, \quad D_{X}^{(H)} J=0, \quad \forall X \in \mathscr{X}(T \tilde{M}) .
$$

By a straightforward calculation, we can prove that a d-connection $D \Gamma=\left(L_{j k}^{i}, L_{j k}^{i}, C_{j c}^{i}\right.$, $C_{j c}^{i}$ ) with the coefficients defined by

$$
D_{\delta_{i}}^{(H)} \delta_{j}=L_{j k}^{i} \delta_{i}, \quad D_{\delta_{i}}^{(H)} \dot{\partial}_{j}=L_{j k}^{i} \dot{\partial}_{i}, \quad D_{\delta_{i}}^{(H)} \delta_{j}=C_{j k}^{i} \delta_{i}, \quad D_{\delta_{i}}^{(H)} \dot{\partial}_{j}=C_{j k}^{i} \dot{\partial}_{i},
$$

where $L_{j k}^{i}$ and $C_{a b}^{e} \rightarrow C_{j k}^{i}$ on $T M$ are defined by the formulas (3.34), defines a torsionless (see the next section on torsion structures) metric d-connection which satisfies the compatibility conditions (3.26).

Almost complex structures and almost Kähler models of Finsler, Lagrange, Hamilton, and Cartan geometries (of first higher orders) are investigated in detail in [45, 49, 50, $51,52,92]$.

3.5. Torsions and curvatures. We outline the basic definitions and formulas for the torsion and curvature structures in v-bundles and cv-bundles provided with $\mathrm{N}$ connection structure.

3.5.1. N-connection curvature. (1) The curvature $\Omega$ of a nonlinear connection $\mathbf{N}$ in a v-bundle $\mathscr{E}$ can be defined in local form as (see $[46,47,48]$ )

$$
\Omega=\frac{1}{2} \Omega_{i j}^{a} d^{i} \wedge d^{j} \otimes \partial_{a},
$$

where

$$
\Omega_{i j}^{a}=\delta_{j} N_{i}^{a}-\delta_{i} N_{j}^{a}=\partial_{j} N_{i}^{a}-\partial_{i} N_{j}^{a}+N_{i}^{b} N_{b j}^{a}-N_{j}^{b} N_{b i}^{a},
$$

$N_{b i}^{a}$ being that from (3.31). 
(2) For the curvature $\breve{\Omega}$ of a nonlinear connection $\breve{N}$ in a cv-bundle $\breve{\mathscr{E}}$, we introduce

$$
\breve{\Omega}=\frac{1}{2} \breve{\Omega}_{i j a} d^{i} \wedge d^{j} \otimes \breve{\partial}^{a},
$$

where

$$
\begin{aligned}
\breve{\Omega}_{i j a}=-\breve{\delta}_{j} \breve{N}_{i a}+\breve{\delta}_{i} \breve{N}_{j a} & =-\partial_{j} \breve{N}_{i a}+\partial_{i} \breve{N}_{j a}+\breve{N}_{i b} \breve{N}_{j a}^{b}-\breve{N}_{j b} \breve{N}_{j a}^{b}, \\
\breve{N}_{j a}^{b} & =\breve{\partial}^{b} \breve{N}_{j a}=\frac{\partial \breve{N}_{j a}}{\partial p_{b}} .
\end{aligned}
$$

(3) The curvatures $\tilde{\Omega}$ of different types of nonlinear connections $\tilde{\mathbf{N}}$ in higher-order anisotropic bundles were analyzed for higher-order tangent/dual tangent bundles and higher-order prolongations of generalized Finsler, Lagrange, and Hamilton spaces in $[45,49,50,51,52]$ and for higher-order anisotropic superspaces and spinor bundles in $[82,83,90,91,92,93]$. For every higher-order anisotropy shell, we will define the coefficients (3.46) or (3.48) depending on the fact what type of subfiber we are considering (a vector or covector fiber).

3.5.2. d-torsions in $\mathbf{v}$ - and cv-bundles. The torsion $\mathbf{T}$ of a d-connection $\mathbf{D}$ in $\mathbf{v}$-bundle $\mathscr{E}$ (cv-bundle $\breve{\mathscr{C}}$ ) is defined by the equation

$$
\mathbf{T}(\mathbf{X}, \mathbf{Y})=\mathbf{X Y} \mathbf{Y}_{\circ}^{\circ} \mathbf{T} \doteq D_{X} \mathbf{Y}-D_{Y} \mathbf{X}-[\mathbf{X}, \mathbf{Y}]
$$

The following h- and v-decompositions hold:

$$
\mathbf{T}(\mathbf{X}, \mathbf{Y})=\mathbf{T}(\mathbf{h X}, \mathbf{h Y})+\mathbf{T}(\mathbf{h X}, \mathbf{v Y})+\mathbf{T}(\mathbf{v X}, \mathbf{h Y})+\mathbf{T}(\mathbf{v X}, \mathbf{v Y})
$$

We consider the projections

$$
\text { hT }(X, Y), \mathbf{v T}(\mathbf{h X}, \mathbf{h Y}), \mathbf{h T}(\mathbf{h X}, \mathbf{h Y}), \ldots
$$

and say that, for instance, $\mathbf{h T}(\mathbf{h X}, \mathbf{h Y})$ is the $\mathrm{h}(\mathrm{h}, \mathrm{h})$-torsion of $\mathbf{D}, \mathbf{v T}(\mathbf{h X}, \mathbf{h Y})$ is the $\mathbf{v}(\mathbf{h}, \mathrm{h})$ torsion of $\mathbf{D}$, and so on.

The torsion (3.49) in v-bundle is locally determined by five d-tensor fields, torsions, defined as

$$
\begin{gathered}
T_{j k}^{i}=\mathbf{h T}\left(\delta_{k}, \delta_{j}\right) \cdot d^{i}, \quad T_{j k}^{a}=\mathbf{v T}\left(\delta_{k}, \delta_{j}\right) \cdot \delta^{a}, \\
P_{j b}^{i}=\mathbf{h T}\left(\partial_{b}, \delta_{j}\right) \cdot d^{i}, \quad P_{j b}^{a}=\mathbf{v T}\left(\partial_{b}, \delta_{j}\right) \cdot \delta^{a}, \quad S_{b c}^{a}=\mathbf{v T}\left(\partial_{c}, \partial_{b}\right) \cdot \delta^{a} .
\end{gathered}
$$

Using formulas (2.29), (2.30), (3.46), and (3.49), we can compute [46, 47, 48] in explicit form the components of torsions (3.52) for a d-connection of type (3.9) and (3.10):

$$
\begin{gathered}
T_{. j k}^{i}=T_{j k}^{i}=L_{j k}^{i}-L_{k j}^{i}, \quad T_{j a}^{i}=C_{. j a}^{i}, \quad T_{a j}^{i}=-C_{j a}^{i}, \\
T_{. j a}^{i}=0, \quad T_{. i b}^{a}=-P_{. b i}^{a}, \\
T_{. b c}^{a}=S_{. b c}^{a}=C_{b c}^{a}-C_{c b}^{a}, \quad T_{. i j}^{a}=\delta_{j} N_{i}^{a}-\delta_{j} N_{j}^{a}, \quad T_{. b i}^{a}=P_{. b i}^{a}=\partial_{b} N_{i}^{a}-L_{. b j}^{a} .
\end{gathered}
$$


Formulas similar to (3.52) and (3.53) hold for cv-bundles:

$$
\begin{gathered}
\check{T}_{j k}^{i}=\mathbf{h T}\left(\delta_{k}, \delta_{j}\right) \cdot d^{i}, \quad \check{T}_{j k a}=\mathbf{v T}\left(\delta_{k}, \delta_{j}\right) \cdot \check{\delta}_{a}, \\
\check{P}_{j}^{i b}=\mathbf{h T}\left(\check{\partial}^{b}, \delta_{j}\right) \cdot d^{i}, \quad \check{P}_{a j}^{b}=\mathbf{v T}\left(\check{\partial}^{b}, \delta_{j}\right) \cdot \check{\delta}_{a}, \quad \check{S}_{a}^{b c}=\mathbf{v T}\left(\check{\partial}^{c}, \check{\partial}^{b}\right) \cdot \check{\delta}_{a}, \\
\check{T}_{. j k}^{i}=\check{T}_{j k}^{i}=L_{j k}^{i}-L_{k j}^{i}, \quad \check{T}_{j}^{i a}=\check{C}_{. j}^{i a}, \quad \check{T}_{j}^{i a}=-\check{C}_{j}^{i a}, \\
\check{T}_{. j}^{i a}=0, \quad \check{T}_{a b}^{j}=-\check{P}_{a b}^{j}, \\
\check{T}_{a}^{b c}=\check{S}_{a}^{b c}=\check{C}_{a}^{b c}-\check{C}_{a}^{c b}, \quad \check{T}_{. i j a}=-\delta_{j} \check{N}_{i a}+\delta_{j} \check{N}_{j a}, \\
\check{T}_{a}^{b i}=\check{P}_{a}^{b i}=-\check{\partial}^{b} \check{N}_{i a}-\check{L}_{a}^{b i} .
\end{gathered}
$$

The formulas for torsion can be generalized for hvc-bundles (on every shell we must write (3.53) or (3.55) depending on the type of shell, vector or co-vector one, we are dealing with).

3.5.3. d-curvatures in $\mathbf{v}$ - and $\mathbf{c v}$-bundles. The curvature $\mathbf{R}$ of a d-connection in $\mathbf{v}$ bundle $\mathscr{E}$ is defined by the equation

$$
\mathbf{R}(X, Y) Z=X Y \bullet R \bullet Z=D_{X} D_{Y} Z-D_{Y} D_{X} Z-D_{[X, Y]} Z \text {. }
$$

The next properties for the h- and v-decompositions of curvature hold:

$$
\begin{gathered}
\mathbf{v R}(X, Y) h Z=0, \quad \mathbf{h R}(X, Y) v Z=0, \\
\mathbf{R}(X, Y) Z=\mathbf{h R}(X, Y) h Z+\mathbf{v R}(X, Y) v Z .
\end{gathered}
$$

From (3.57) and the equation $\mathbf{R}(\mathbf{X}, \mathbf{Y})=-\mathbf{R}(\mathbf{Y}, \mathbf{X})$, we get that the curvature of a dconnection $\mathbf{D}$ in $\mathscr{E}$ is completely determined by the following six d-tensor fields:

$$
\begin{aligned}
R_{h . j k}^{i} & =d^{i} \cdot \mathbf{R}\left(\delta_{k}, \delta_{j}\right) \delta_{h}, & & R_{b . j k}^{a}=\delta^{a} \cdot \mathbf{R}\left(\delta_{k}, \delta_{j}\right) \partial_{b}, \\
P_{j . k c}^{. i} & =d^{i} \cdot \mathbf{R}\left(\partial_{c}, \partial_{k}\right) \delta_{j}, & & P_{b . k c}^{a}=\delta^{a} \cdot \mathbf{R}\left(\partial_{c}, \partial_{k}\right) \partial_{b}, \\
S_{j . b c}^{. i} & =d^{i} \cdot \mathbf{R}\left(\partial_{c}, \partial_{b}\right) \delta_{j}, & & S_{b . c d}^{a}=\delta^{a} \cdot \mathbf{R}\left(\partial_{d}, \partial_{c}\right) \partial_{b} .
\end{aligned}
$$

By a direct computation, using (2.29), (2.30), (3.9), (3.10), and (3.58), we get

$$
\begin{aligned}
R_{h . j k}^{. i} & =\delta_{h} L_{. h j}^{i}-\delta_{j} L_{. h k}^{i}+L_{. h j}^{m} L_{m k}^{i}-L_{. h k}^{m} L_{m j}^{i}+C_{. h a}^{i} R_{. j k}^{a}, \\
R_{b . j k}^{. a} & =\delta_{k} L_{. b j}^{a}-\delta_{j} L_{. b k}^{a}+L_{. b j}^{c} L_{. c k}^{a}-L_{. b k}^{c} L_{. c j}^{a}+C_{. b c}^{a} R_{. j k}^{c}, \\
P_{j . k a}^{. i} & =\partial_{a} L_{. j k}^{i}-\left(\delta_{k} C_{. j a}^{i}+L_{. l k}^{i} C_{. j a}^{l}-L_{. j k}^{l} C_{. l a}^{i}-L_{. a k}^{c} C_{. j c}^{i}\right)+C_{. j b}^{i} P_{. k a}^{b}, \\
P_{b . k a}^{c} & =\partial_{a} L_{. b k}^{c}-\left(\delta_{k} C_{. b a}^{c}+L_{. d k}^{c} C_{. b a}^{d}-L_{. b k}^{d} C_{. d a}^{c}-L_{. a k}^{d} C_{. b d}^{c}\right)+C_{. b d}^{c} P_{. k a}^{d}, \\
S_{j . b c}^{. i} & =\partial_{c} C_{. j b}^{i}-\partial_{b} C_{. j c}^{i}+C_{. j b}^{h} C_{. h c}^{i}-C_{. j c}^{h} C_{h b}^{i}, \\
S_{b . c d}^{a} & =\partial_{d} C_{. b c}^{a}-\partial_{c} C_{. b d}^{a}+C_{. b c}^{e} C_{. e d}^{a}-C_{. b d}^{e} C_{. e c}^{a} .
\end{aligned}
$$

We note that d-torsions (3.53) and d-curvatures (3.59) are computed in explicit form by particular cases of d-connections (3.33), (3.36), and (3.38). 
For cv-bundles, we have

$$
\begin{aligned}
& \check{R}_{h . j k}^{i}=d^{i} \cdot \mathbf{R}\left(\delta_{k}, \delta_{j}\right) \delta_{h}, \quad \check{R}_{a . j k}^{b}=\check{\delta}_{a} \cdot \mathbf{R}\left(\delta_{k}, \delta_{j}\right) \check{\partial}^{b}, \\
& \check{P}_{j . k}^{. i c}=d^{i} \cdot \mathbf{R}\left(\check{\partial}^{c}, \partial_{k}\right) \delta_{j}, \quad \check{P}_{a . k}^{b c}=\check{\delta}_{a} \cdot \mathbf{R}\left(\check{\partial}^{c}, \partial_{k}\right) \check{\partial}^{b}, \\
& \check{S}_{j .}^{i b c}=d^{i} \cdot \mathbf{R}\left(\check{\partial}^{c}, \breve{\partial}^{b}\right) \delta_{j}, \quad \check{S}_{. a}^{b . c d}=\check{\delta}_{a} \cdot \mathbf{R}\left(\check{\partial}^{d}, \check{\partial ̆}^{c}\right) \check{\partial ̆}^{b}, \\
& \check{R}_{h . j k}^{i}=\check{\delta}_{h} L_{. h j}^{i}-\check{\delta}_{j} L_{. h k}^{i}+L_{. h j}^{m} L_{m k}^{i}-L_{. h k}^{m} L_{m j}^{i}+C_{. h}^{i a} \check{R}_{. a j k}, \\
& \check{R}_{. a j k}^{b .}=\check{\delta}_{k} \check{L}_{a . j}^{b}-\check{\delta}_{j} \check{L}_{b k}^{a}+\check{L}_{c j}^{b} \check{L}_{. a k}^{c}-\check{L}_{c k}^{b} \check{L}_{a . j}^{c}+\check{C}_{a}^{b c} \check{R}_{c . j k} \text {, } \\
& \check{P}_{j . k}^{. i a}=\check{\partial}^{a} L_{. j k}^{i}-\left(\check{\delta}_{k} \check{C}_{. j}^{i a}+L_{. l k}^{i} \check{C}_{. j}^{l a}-L_{. j k}^{l} \check{C}_{. l}^{i a}-\check{L}_{c k}^{a} \check{C}_{. j}^{i c}\right)+\check{C}_{. j}^{i b} \check{P}_{b k}^{a} \text {, } \\
& \check{P}_{c k}^{b a}=\check{\partial}^{a} \check{L}_{c . k}^{b}-\left(\check{\delta}_{k} \check{C}_{c .}^{b a}+\check{L}_{c . k}^{b d} \check{C}_{d}^{b a}-\check{L}_{d . k}^{b} \check{C}_{c .}^{a d}\right)-\check{L}_{d k}^{a} \check{C}_{c .}^{b d}+\check{C}_{c .}^{b d} \check{P}_{d . k}^{a} \text {, } \\
& \check{S}_{j .}^{. i b c}=\check{\partial}^{c} \check{C}_{. j}^{i b}-\check{\partial}^{b} \check{C}_{. j}^{i c}+\check{C}_{. j}^{h b} \check{C}_{. h}^{i c}-\check{C}_{. j}^{h c} \check{C}_{h}^{i b} \text {, } \\
& \check{S}_{a .}^{b c d}=\check{\partial}^{d} \check{C}_{a .}^{b c}-\check{\partial}^{c} \check{C}_{a .}^{b d}+\check{C}_{e .}^{b c} \check{C}_{a .}^{e d}-\check{C}_{e .}^{b d} \check{C}_{. a}^{e c} .
\end{aligned}
$$

The formulas for curvature can also be generalized for hvc-bundles (on every shell we must write (3.53) or (3.54) depending on the type of shell, vector or covector one, we are dealing with).

4. Generalizations of Finsler geometry. We outline the basic definitions and formulas for Finsler, Lagrange, and generalized Lagrange spaces (constructed on tangent bundle) and for Cartan, Hamilton, and generalized Hamilton spaces (constructed on cotangent bundle). The original results are given in detail in [45, 46, 47, 48, 49, 50, 51, 52], see also developments for superbundles in [90, 91, 92].

4.1. Finsler spaces. The Finsler geometry is modeled on tangent bundle TM.

DeFINITION 4.1. A Finsler space (manifold) is a pair $F^{n}=(M, F(x, y))$, where $M$ is a real $n$-dimensional differentiable manifold and $F: T M \rightarrow \mathbb{R}$ is a scalar function which satisfies the following conditions:

(1) $F$ is a differentiable function on the manifold $\widetilde{T M}=T M \backslash\{0\}$ and $F$ is continuous on the null section of the projection $\pi: T M \rightarrow M$;

(2) $F$ is a positive function, homogeneous on the fibers of the $T M$, that is, $F(x, \lambda y)=$ $\lambda F(x, y), \lambda \in \mathbb{R}$

(3) The Hessian of $F^{2}$ with elements

$$
g_{i j}^{(F)}(x, y)=\frac{1}{2} \frac{\partial^{2} F^{2}}{\partial y^{i} \partial y^{j}}
$$

is positively defined on $\widetilde{T M}$.

The function $F(x, y)$ and $g_{i j}(x, y)$ are called, respectively, the fundamental function and the fundamental (or metric) tensor of the Finsler space $F$.

One considers "anisotropic" (depending on directions $y^{i}$ ) Christoffel symbols. For simplicity, we write $g_{i j}^{(F)}=g_{i j}$,

$$
\gamma_{j k}^{i}(x, y)=\frac{1}{2} g^{i r}\left(\frac{\partial g_{r k}}{\partial x^{j}}+\frac{\partial g_{j r}}{\partial x^{k}}-\frac{\partial g_{j k}}{\partial x^{r}}\right),
$$


which are used for the definition of the Cartan N-connection

$$
N_{(c) j}^{i}=\frac{1}{2} \frac{\partial}{\partial y^{j}}\left[\gamma_{n k}^{i}(x, y) y^{n} y^{k}\right]
$$

This N-connection can be used for the definition of an almost complex structure like in (3.39) and to define on $T M$ a d-metric

$$
\mathbf{G}_{(F)}=g_{i j}(x, y) d x^{i} \otimes d x^{j}+g_{i j}(x, y) \delta y^{i} \otimes \delta y^{j},
$$

with $g_{i j}(x, y)$ taken as in (4.1).

Using the Cartan N-connection (4.3) and Finsler metric tensor (4.1) (or, equivalently, the d-metric (4.4)), we can introduce the canonical d-connection

$$
D \Gamma\left(N_{(c)}\right)=\Gamma_{(c) \beta \gamma}^{\alpha}=\left(L_{(c) j k}^{i}, C_{(c) j k}^{i}\right)
$$

with the coefficients computed as in (3.44) and (3.34) with $h_{a b} \rightarrow g_{i j}$. The d-connection $D \Gamma\left(N_{(c)}\right)$ has the unique property that it is torsionless and satisfies the metricity conditions for both the horizontal and vertical components, that is, $D_{\alpha} g_{\beta \gamma}=0$.

The d-curvatures

$$
\check{R}_{h . j k}^{i}=\left\{\check{R}_{h . j k}^{i}, \check{P}_{j . k}^{. i l}, S_{(c) j . k l}^{i}\right\}
$$

on a Finsler space provided with Cartan N-connection and Finsler metric structures are computed following the formulas (3.59) when the $a, b, c, \ldots$ indices are identified with $i, j, k, \ldots$ indices. It should be emphasized that in this case all values $g_{i j}, \Gamma_{(c) \beta \gamma}^{\alpha}$, and $R_{(c) \beta . \gamma \delta}^{\alpha}$ are defined by a fundamental function $F(x, y)$.

In general, we can consider that a Finsler space is provided with a metric $g_{i j}=$ $\partial^{2} F^{2} / 2 \partial y^{i} \partial y^{j}$, but the N-connection and d-connection are defined in a different manner; they are not even determined by $F$.

4.2. Lagrange and generalized Lagrange spaces. The notion of Finsler spaces was generalized by Kern [30] and Miron [38, 39]. It is widely developed in [46, 47, 48] and extended to superspaces in [76, 77, 78, 81, 90, 91, 92].

The idea of extension was to consider instead of the homogeneous fundamental function $F(x, y)$ in a Finsler space a more general one, a Lagrangian $L(x, y)$ defined as a differentiable mapping $L:(x, y) \in T M \rightarrow L(x, y) \in \mathbb{R}$, of class $C^{\infty}$ on manifold $\widetilde{T M}$, and continuous on the null section $0: M \rightarrow T M$ of the projection $\pi: T M \rightarrow M$. A Lagrangian is regular if it is differentiable and the Hessian

$$
g_{i j}^{(L)}(x, y)=\frac{1}{2} \frac{\partial^{2} L^{2}}{\partial y^{i} \partial y^{j}}
$$

is of rank $n$ on $M$.

DEFINITION 4.2. A Lagrange space is a pair $L^{n}=(M, L(x, y))$, where $M$ is a smooth real $n$-dimensional manifold provided with regular Lagrangian $L(x, y)$ structure $L$ : $T M \rightarrow \mathbb{R}$ for which $g_{i j}(x, y)$ from $(4.7)$ has a constant signature over the manifold $\widetilde{T M}$. 
The fundamental Lagrange function $L(x, y)$ defines a canonical $\mathrm{N}$-connection

$$
N_{(c L) j}^{i}=\frac{1}{2} \frac{\partial}{\partial y^{j}}\left[g^{i k}\left(\frac{\partial^{2} L^{2}}{\partial y^{k} \partial y^{h}} y^{h}-\frac{\partial L}{\partial x^{k}}\right)\right]
$$

as well as a d-metric

$$
\mathbf{G}_{(L)}=g_{i j}(x, y) d x^{i} \otimes d x^{j}+g_{i j}(x, y) \delta y^{i} \otimes \delta y^{j},
$$

with $g_{i j}(x, y)$ taken as in (4.7). We can also introduce an almost Kähler structure and an almost Hermitian model of $L^{n}$, denoted as $H^{2 n}$ as in the case of Finsler spaces but with a proper fundamental Lagrange function and metric tensor $g_{i j}$. The canonical metric d-connection $D \Gamma\left(N_{(c L)}\right)=\Gamma_{(c L) \beta y}^{\alpha}=\left(L_{(c L) j k}^{i}, C_{(c L) j k}^{i}\right)$ is to be computed by the same formulas (3.44) and (3.34) with $h_{a b} \rightarrow g_{i j}^{(L)}$, for $N_{(c L) j}^{i}$. The d-torsions (3.53) and d-curvatures (3.59) are defined, in this case, by $L_{(c L) j k}^{i}$ and $C_{(c L) j k}^{i}$. We also note that instead of $N_{(c L) j}^{i}$ and $\Gamma_{(c L) \beta \gamma}^{\alpha}$ one can consider on an $L^{n}$-space arbitrary $\mathrm{N}$-connections $N_{j}^{i}$, d-connections $\Gamma_{\beta \gamma}^{\alpha}$, which are not defined only by $L(x, y)$ and $g_{i j}^{(L)}$ but can be metric or nonmetric with respect to the Lagrange metric.

The next step of generalization is to consider an arbitrary metric $g_{i j}(x, y)$ on $T M$ instead of (4.7) which is the second derivative of "anisotropic" coordinates $y^{i}$ of a Lagrangian [38, 39].

DEFINITION 4.3. A generalized Lagrange space is a pair $\mathrm{GL}^{n}=\left(M, g_{i j}(x, y)\right)$, where $g_{i j}(x, y)$ is a covariant, symmetric d-tensor field of rank $n$ and of constant signature on $\widetilde{T M}$.

One can consider different classes of $\mathrm{N}$ - and d-connections on $T M$, which are compatible (metric) or noncompatible with (4.9) for arbitrary $g_{i j}(x, y)$. We can apply all formulas for d-connections, $\mathrm{N}$-curvatures, d-torsions, and d-curvatures as in a v-bundle $\mathscr{E}$, but reconsidering them on $T M$, by changing $h_{a b} \rightarrow g_{i j}(x, y)$ and $N_{i}^{a} \rightarrow N_{i}^{k}$.

4.3. Cartan spaces. The theory of Cartan spaces (see, e.g., [29, 66]) was formulated in a new fashion in Miron's works [40,42] by considering them as duals to the Finsler spaces (see details and references in [45, 49, 50, 51, 52]). Roughly, a Cartan space is constructed on a cotangent bundle $T^{*} M$ like a Finsler space on the corresponding tangent bundle $T M$.

Consider a real smooth manifold $M$, the cotangent bundle $\left(T^{*} M, \pi^{*}, M\right)$, and the manifold $\widetilde{T^{*} M}=T^{*} M \backslash\{0\}$.

DEFINITION 4.4. A Cartan space is a pair $C^{n}=(M, K(x, p))$ such that $K: T^{*} M \rightarrow \mathbb{R}$ is a scalar function which satisfies the following conditions:

(1) $K$ is a differentiable function on the manifold $\widetilde{T^{*} M}=T^{*} M \backslash\{0\}$ and is continuous on the null section of the projection $\pi^{*}: T^{*} M \rightarrow M$;

(2) $K$ is a positive function, homogeneous on the fibers of the $T^{*} M$, that is, $K(x, \lambda p)$ $=\lambda F(x, p), \lambda \in \mathbb{R} ;$ 
(3) the Hessian of $K^{2}$ with elements

$$
\check{\mathfrak{g}}_{(K)}^{i j}(x, p)=\frac{1}{2} \frac{\partial^{2} K^{2}}{\partial p_{i} \partial p_{j}}
$$

is positively defined on $\widetilde{T^{*} M}$.

The function $K(x, y)$ and $\check{g}^{i j}(x, p)$ are called, respectively, the fundamental function and the fundamental (or metric) tensor of the Cartan space $C^{n}$. We use symbols like " $\breve{g}$ " to emphasize that the geometrical objects are defined on a dual space.

We consider "anisotropic" (depending on directions, momenta, $p_{i}$ ) Christoffel symbols; for simplicity, we write the inverse to (4.10) as $g_{i j}^{(K)}=\breve{g}_{i j}$,

$$
\check{\gamma}_{j k}^{i}(x, p)=\frac{1}{2} \check{g}^{i r}\left(\frac{\partial \check{g}_{r k}}{\partial x^{j}}+\frac{\partial \check{g}_{j r}}{\partial x^{k}}-\frac{\partial \check{g}_{j k}}{\partial x^{r}}\right),
$$

which are used for the definition of the canonical N-connection,

$$
\check{N}_{i j}=\check{\gamma}_{i j}^{k} p_{k}-\frac{1}{2} \gamma_{n l}^{k} p_{k} p^{l} \breve{\partial}^{n} \check{\check{g}}_{i j}, \quad \check{\partial}^{n}=\frac{\partial}{\partial p_{n}} .
$$

This N-connection can be used to define an almost complex structure like in (3.39) and to define on $T^{*} M$ a d-metric

$$
\check{\mathbf{G}}_{(k)}=\check{\mathfrak{g}}_{i j}(x, p) d x^{i} \otimes d x^{j}+\check{\mathfrak{g}}^{i j}(x, p) \delta p_{i} \otimes \delta p_{j},
$$

with $\check{g}^{i j}(x, p)$ taken as in (4.10).

Using the canonical N-connection (4.12) and Finsler metric tensor (4.10) (or, equivalently, the d-metric (4.13)), we can introduce the canonical d-connection

$$
D \check{\Gamma}\left(\check{N}_{(k)}\right)=\check{\Gamma}_{(k) \beta \gamma}^{\alpha}=\left(\check{H}_{(k) j k}^{i}, \check{C}_{(k) i}^{j k}\right)
$$

with the coefficients

$$
\check{H}_{(k) j k}^{i}=\frac{1}{2} \check{\mathfrak{g}}^{i r}\left(\check{\delta}_{j} \check{\mathfrak{g}}_{r k}+\check{\delta}_{k} \check{\mathfrak{g}}_{j r}-\check{\delta}_{r} \check{\mathfrak{g}}_{j k}\right), \quad \check{C}_{(k) i}^{j k}=\check{\mathfrak{g}}_{i s} \breve{\partial}^{s} \check{\mathfrak{g}}^{j k}
$$

The d-connection $D \check{\Gamma}\left(\check{N}_{(k)}\right)$ has the unique property that it is torsionless and satisfies the metricity conditions for both the horizontal and vertical components, that is, $\check{D}_{\alpha} \check{\mathfrak{g}}_{\beta \gamma}=0$.

The d-curvatures

$$
\check{R}_{(k) \beta . \gamma \delta}^{. \alpha}=\left\{R_{(k) h . j k}^{. i}, P_{(k) j . k m}^{i}, \check{S}_{j}^{i k l}\right\}
$$

on a Finsler space provided with Cartan N-connection and Finsler metric structures are computed following formulas (3.61) when the $a, b, c, \ldots$ indices are identified with the $i, j, k, \ldots$ indices. It should be emphasized that in this case all values $\check{\mathfrak{g}}_{i j}, \check{\Gamma}_{(k) \beta \gamma}^{\alpha}$, and $\check{R}_{(k) \beta . \gamma \delta}^{. \alpha}$ are defined by a fundamental function $K(x, p)$.

In general, we can consider that a Cartan space is provided with a metric $\check{g}^{i j}=$ $\partial^{2} K^{2} / 2 \partial p_{i} \partial p_{j}$, but the $\mathrm{N}$-connection and d-connection could be defined in a different manner, even if they are not determined by $K$. 
4.4. Generalized Hamilton and Hamilton spaces. The geometry of Hamilton spaces was defined and investigated by Miron in [41, 43, 44] (see details and references in $[45,49,50,51,52])$. It was developed on the cotangent bundle as a dual geometry to the geometry of Lagrange spaces. Here, we start with the definition of generalized Hamilton spaces and then consider the particular case.

DEFINITION 4.5. A generalized Hamilton space is a pair $G H^{n}=\left(M, \breve{g}^{i j}(x, p)\right)$, where $M$ is a real $n$-dimensional manifold and $\breve{g}^{i j}(x, p)$ is a contravariant, symmetric, nondegenerate tensor of rank $n$ and of constant signature on $\widetilde{T^{*} M}$.

The value $\breve{g}^{i j}(x, p)$ is called the fundamental (or metric) tensor of the space $G H^{n}$. One can define such values for every paracompact manifold $M$. In general, an N-connection on $G H^{n}$ is not determined by $\breve{g}^{i j}$. Therefore, we can consider arbitrary coefficients $\check{N}_{i j}(x, p)$ and define on $T^{*} M$ a d-metric like (3.27)

$$
\breve{\mathbf{G}}=\breve{g}_{\alpha \beta}(\breve{u}) \breve{\delta}^{\alpha} \otimes \breve{\delta}^{\beta}=\breve{g}_{i j}(\breve{u}) d^{i} \otimes d^{j}+\breve{g}^{i j}(\breve{u}) \breve{\delta}_{i} \otimes \breve{\delta}_{j} .
$$

These N-coefficients $\check{N}_{i j}(x, p)$ and d-metric structure (4.17) allow to define an almost Kähler model of generalized Hamilton spaces and to define canonical d-connections, d-torsions, and d-curvatures (see, respectively, formulas (3.34), (3.36), (3.55), and (3.59) with the fiber coefficients redefined for the cotangent bundle $\left.T^{*} M\right)$.

A generalized Hamilton space $G H^{n}=\left(M, \breve{g}^{i j}(x, p)\right)$ is called reducible to a Hamilton one if there exists a Hamilton function $H(x, p)$ on $T^{*} M$ such that

$$
\check{g}^{i j}(x, p)=\frac{1}{2} \frac{\partial^{2} H}{\partial p_{i} \partial p_{j}} .
$$

DEFINITION 4.6. A Hamilton space is a pair $H^{n}=(M, H(x, p))$ such that $H: T^{*} M \rightarrow$ $\mathbb{R}$ is a scalar function which satisfies the following conditions:

(1) $H$ is a differentiable function on the manifold $\widetilde{T^{*} M}=T^{*} M \backslash\{0\}$ and is continuous on the null section of the projection $\pi^{*}: T^{*} M \rightarrow M$;

(2) the Hessian of $H$ with elements (4.18) is positively defined on $\widetilde{T^{*} M}$ and $\check{g}^{i j}(x, p)$ is a nondegenerate matrix of rank $n$ and of constant signature.

For Hamilton spaces, the canonical N-connection (defined by $H$ and its Hessian) exists,

$$
\check{N}_{i j}=\frac{1}{4}\left\{\check{\mathfrak{g}}_{i j}, H\right\}-\frac{1}{2}\left(\check{\mathfrak{g}}_{i k} \frac{\partial^{2} H}{\partial p_{k} \partial x^{j}}+\check{\mathfrak{g}}_{j k} \frac{\partial^{2} H}{\partial p_{k} \partial x^{i}}\right),
$$

where the Poisson brackets, for arbitrary functions $f$ and $g$ on $T^{*} M$, act as

$$
\{f, g\}=\frac{\partial f}{\partial p_{i}} \frac{\partial g}{\partial x^{i}}-\frac{\partial g}{\partial p_{i}} \frac{\partial p}{\partial x^{i}} .
$$

The canonical d-connection $D \check{\Gamma}\left(\check{N}_{(c)}\right)=\check{\Gamma}_{(c) \beta \gamma}^{\alpha}=\left(\check{H}_{(c) j k}^{i}, \check{C}_{(c) i}^{j k}\right)$ is defined by the coefficients

$$
\check{H}_{(c) j k}^{i}=\frac{1}{2} \check{\mathfrak{g}}^{i s}\left(\check{\delta}_{j} \check{\mathfrak{g}}_{s k}+\check{\delta}_{k} \check{\mathfrak{g}}_{j s}-\check{\delta}_{s} \check{\mathfrak{g}}_{j k}\right), \quad \check{C}_{(c) i}^{j k}=-\frac{1}{2} \check{\mathfrak{g}}_{i s} \check{\partial}^{j} \check{\mathfrak{g}}^{s k}
$$


In result we can compute the d-torsions and d-curvatures like on cv-bundle or on Cartan spaces. On Hamilton spaces, all such objects are defined by the Hamilton function $H(x, p)$ and indices have to be reconsidered for cofibers of the cotangent bundle.

5. Clifford bundles and N-connections. The theory of anisotropic spinors was extended on higher-order anisotropic (ha) spaces [92, 93, 106]. In brief, such spinors will be called ha-spinors which are defined as some Clifford ha-structures defined with respect to a distinguished quadratic form (3.28) on an hvc-bundle. For simplicity, the bulk of formulas will be given with respect to higher-order vector bundles. To rewrite such formulas for hvc-bundles is to consider for the "dual" shells of higher-order anisotropy some dual vector spaces and associated dual spinors.

5.1. Distinguished Clifford algebras. The typical fiber of dv-bundle $\xi_{d}, \pi_{d}: H E \oplus$ $V_{1} E \oplus \cdots \oplus V_{z} E \rightarrow E$, is a d-vector space, $\mathscr{F}=h \mathscr{F} \oplus v_{1} \mathscr{F} \oplus \cdots \oplus v_{z} \mathscr{F}$, split into horizontal $h \mathscr{F}$ and vertical $v_{p} \mathscr{F}, p=1, \ldots, z$, subspaces, with a bilinear quadratic form $G(g, h)$ induced by an hvc-bundle metric (3.28). Clifford algebras (see, e.g., [28, 61, 62, 63]) formulated for d-vector spaces will be called Clifford d-algebras [88, 89, 101]. We will consider the main properties of Clifford d-algebras. The proof of the theorems will be based on the technique developed in [28, 92, 106], correspondingly adapted to the distinguished character of spaces in consideration.

Let $k$ be a number field (for our purposes $k=\mathbb{R}$ or $k=\mathbb{C}, \mathbb{R}$ and $\mathbb{C}$ are, respectively, real and complex number fields) and define $\mathscr{F}$, as a d-vector space, on $k$ provided with nondegenerate symmetric quadratic form (metric) $G$. Let $C$ be an algebra on $k$ (not necessarily commutative) and $j: \mathscr{F} \rightarrow C$ a homomorphism of underlying vector spaces such that $j(u)^{2}=G(u) \cdot 1$ ( 1 is the unity in algebra $C$ and d-vector $u \in \mathscr{F}$ ). We are interested in the definition of the pair $(C, j)$ satisfying the next universality conditions. For every $k$-algebra $A$ and arbitrary homomorphism $\varphi: \mathscr{F} \rightarrow A$ of the underlying d-vector spaces, such that $(\varphi(u))^{2} \rightarrow G(u) \cdot 1$, there is a unique homomorphism of algebras $\psi: C \rightarrow A$ defined as commutative diagrams.

The algebra solving this problem will be denoted as $C(\mathscr{F}, A)$ (equivalently as $C(G)$ or $C(\mathscr{F}))$ and called Clifford d-algebra associated with pair $(\mathscr{F}, G)$.

THEOREM 5.1. There is a unique solution $(C, j)$ up to isomorphism.

Proof. See [82, 83, 92].

Now we reformulate for d-algebras the Chevalley theorem [13].

THEOREM 5.2. The Clifford d-algebra

$$
C\left(h \mathscr{F} \oplus v_{1} \mathscr{F} \oplus \cdots \oplus v_{z} \mathscr{F}, g+h_{1}+\cdots+h_{z}\right)
$$

is naturally isomorphic to $C(g) \otimes C\left(h_{1}\right) \otimes \cdots \otimes C\left(h_{z}\right)$.

Proof. See [82, 83, 92].

From the presented theorems, we conclude that all operations with Clifford dalgebras can be reduced to calculations for $C(h \mathscr{F}, g)$ and $C\left(v_{(p)} \mathscr{F}, h_{(p)}\right)$ which are usual Clifford algebras of dimensions $2^{n}$ and $2^{m_{p}}$, respectively $[6,28]$. 
Of special interest is the case when $k=\mathbb{R}$ and $\mathscr{F}$ is isomorphic to a vector space $\mathbb{R}^{p+q, a+b}$ provided with quadratic form

$$
-x_{1}^{2}-\cdots-x_{p}^{2}+x_{p+q}^{2}-y_{1}^{2}-\cdots-y_{a}^{2}+\cdots+y_{a+b}^{2} .
$$

In this case, the Clifford algebra, denoted as $\left(C^{p, q}, C^{a, b}\right)$, is generated by the symbols $e_{1}^{(x)}, e_{2}^{(x)}, \ldots, e_{p+q}^{(x)}, e_{1}^{(y)}, e_{2}^{(y)}, \ldots, e_{a+b}^{(y)}$ satisfying the properties

$$
\begin{gathered}
\left(e_{i}\right)^{2}=-1 \quad(1 \leq i \leq p), \quad\left(e_{j}\right)^{2}=-1 \quad(1 \leq j \leq a), \\
\left(e_{k}\right)^{2}=1 \quad(p+1 \leq k \leq p+q), \\
\left(e_{j}\right)^{2}=1 \quad(n+1 \leq s \leq a+b), \quad e_{i} e_{j}=-e_{j} e_{i}, \quad i \neq j .
\end{gathered}
$$

Explicit calculations of $C^{p, q}$ and $C^{a, b}$ are possible by using the isomorphisms [28, 61, $62,63]$

$$
C^{p+n, q+n} \simeq C^{p, q} \otimes M_{2}(\mathbb{R}) \otimes \cdots \otimes M_{2}(\mathbb{R}) \cong C^{p, q} \otimes M_{2^{n}}(\mathbb{R}) \cong M_{2^{n}}\left(C^{p, q}\right),
$$

where $M_{S}(A)$ denotes the ring of quadratic matrices of order $s$ with coefficients in ring $A$. Here, we write the simplest isomorphisms $C^{1,0} \simeq \mathbb{C}, C^{0,1} \simeq \mathbb{R} \oplus \mathbb{R}$, and $C^{2,0}=\mathscr{H}$, where $\mathscr{H}$ denotes the body of quaternions.

Now, we emphasize that higher-order Lagrange and Finsler spaces, denoted by $H^{2 n}$ spaces, admit locally a structure of Clifford algebra on complex vector spaces. Really, by using almost Hermitian structure $J_{\alpha}^{\beta}$ and considering complex space $\mathbb{C}^{n}$ with nondegenerate quadratic form $\sum_{a=1}^{n}\left|z_{a}\right|^{2}, z_{a} \in \mathbb{C}^{2}$, induced locally by metric (3.28) (rewritten in complex coordinates as $z_{a}=x_{a}+i y_{a}$ ), we define Clifford algebra

$$
\overleftarrow{C}^{n}=\underbrace{\overleftarrow{C}^{1} \otimes \cdots \otimes \overleftarrow{C}^{1}}_{n}
$$

where $\overleftarrow{C}^{1}=\mathbb{C} \otimes_{R} \mathbb{C}=\mathbb{C} \oplus \mathbb{C}$ or, in consequence, $\overleftarrow{C}^{n} \simeq C^{n, 0} \otimes_{\mathbb{R}} \mathbb{C} \approx C^{0, n} \otimes_{\mathbb{R}} \mathbb{C}$. Explicit calculations lead to isomorphisms

$$
\begin{aligned}
\overleftarrow{C}^{2}=C^{0,2} \otimes_{\mathbb{R}} \mathbb{C} \approx & M_{2}(\mathbb{R}) \otimes_{\mathbb{R}} \mathbb{C} \approx M_{2}\left(\overleftarrow{C}^{n}\right), \quad C^{2 p} \approx M_{2^{p}}(\mathbb{C}), \\
& \overleftarrow{C}^{2 p+1} \approx M_{2 p}(\mathbb{C}) \oplus M_{2}(\mathbb{C}),
\end{aligned}
$$

which show that complex Clifford algebras, defined locally for $H^{2 n}$-spaces, have periodicity 2 on $p$.

Considerations presented in the proof of Theorem 5.1 show that the map $j: \mathscr{F} \rightarrow$ $C(\mathscr{F})$ is monomorphic, so we can identify the space $\mathscr{F}$ with its image in $C(\mathscr{F}, G)$, denoted as $u \rightarrow \bar{u}$, if $u \in C^{(0)}(\mathscr{F}, G)\left(u \in C^{(1)}(\mathscr{F}, G)\right)$; then $u=\bar{u}$ (resp., $\left.\bar{u}=-u\right)$.

Definition 5.3. The set of elements $u \in C(G)^{*}$, where $C(G)^{*}$ denotes the multiplicative group of invertible elements of $C(\mathscr{F}, G)$ satisfying $\bar{u} \mathscr{F} u^{-1} \in \mathscr{F}$, is called the twisted Clifford d-group, denoted as $\widetilde{\Gamma}(\mathscr{F})$.

Let $\tilde{\rho}: \tilde{\Gamma}(\mathscr{F}) \rightarrow \operatorname{GL}(\mathscr{F})$ be the homorphism given by $u \rightarrow \rho \tilde{u}$, where $\tilde{\rho}_{u}(w)=\bar{u} w u^{-1}$. We can verify that ker $\tilde{\rho}=\mathbb{R}^{*}$ is a subgroup in $\widetilde{\Gamma}(\mathscr{F})$. 
The canonical map $j: \mathscr{F} \rightarrow C(\mathscr{F})$ can be interpreted as the linear map $\mathscr{F} \rightarrow C(\mathscr{F})^{0}$ satisfying the universal properties of Clifford d-algebras. This leads to a homomorphism of algebras, $C(\mathscr{F}) \rightarrow C(\mathscr{F})^{t}$, considered by an anti-involution of $C(\mathscr{F})$ and denoted as $u \rightarrow$ ${ }^{t} u$. More exactly, if $u_{1} \cdots u_{n} \in \mathscr{F}$, then $t_{u}=u_{n} \cdots u_{1}$ and ${ }^{t} \bar{u}=\overline{{ }^{t} u}=(-1)^{n} u_{n} \cdots u_{1}$.

DEFINITION 5.4. The spinor norm of arbitrary $u \in C(\mathscr{F})$ is defined as $S(u)={ }^{t} \bar{u} \cdot u \in$ $C(\mathscr{F})$.

It is obvious that if $u, u^{\prime}, u^{\prime \prime} \in \widetilde{\Gamma}(\mathscr{F})$, then $S\left(u, u^{\prime}\right)=S(u) S\left(u^{\prime}\right)$ and $S\left(u u^{\prime} u^{\prime \prime}\right)=$ $S(u) S\left(u^{\prime}\right) S\left(u^{\prime \prime}\right)$. For $u, u^{\prime} \in \mathscr{F}, S(u)=-G(u)$ and $S\left(u, u^{\prime}\right)=S(u) S\left(u^{\prime}\right)=S\left(u u^{\prime}\right)$.

We introduce the orthogonal group $O(G) \subset \mathrm{GL}(G)$ defined by metric $G$ on $\mathscr{F}$ and denote sets

$$
\operatorname{SO}(G)=\{u \in O(G), \operatorname{det}|u|=1\}, \quad \operatorname{Pin}(G)=\{u \in \widetilde{\Gamma}(\mathscr{F}), S(u)=1\},
$$

and $\operatorname{Spin}(G)=\operatorname{Pin}(G) \cap C^{0}(\mathscr{F})$. For $\mathscr{F} \cong \mathbb{R}^{n+m}$, we write $\operatorname{Spin}\left(n_{E}\right)$. By straightforward calculations (see similar considerations in [28]), we can verify the exactness of these sequences:

$$
\begin{gathered}
1 \rightarrow \frac{\mathscr{L}}{2} \longrightarrow \operatorname{Pin}(G) \rightarrow O(G) \longrightarrow 1, \\
1 \longrightarrow \frac{\mathscr{L}}{2} \longrightarrow \operatorname{Spin}(G) \rightarrow \mathrm{SO}(G) \longrightarrow 0, \\
1 \rightarrow \frac{\mathscr{L}}{2} \longrightarrow \operatorname{Spin}\left(n_{E}\right) \longrightarrow \mathrm{SO}\left(n_{E}\right) \longrightarrow 1 .
\end{gathered}
$$

We conclude this subsection by emphasizing that the spinor norm was defined with respect to a quadratic form induced by a metric in dv-bundle $\mathscr{E}^{\langle}\langle z\rangle$. This approach differs from those presented in $[4,57,58,59,74]$.

5.2. Clifford ha-bundles. We will consider two variants of generalization of spinor constructions defined for d-vector spaces to the case of distinguished vector bundle spaces enabled with the structure of $\mathrm{N}$-connection. The first is to use the extension to the category of vector bundles. The second is to define the Clifford fibration associated with compatible linear d-connection and metric $G$ on a dv-bundle. We will analyze both variants.

5.2.1. Clifford d-module structure in $\mathbf{d v}$-bundles. Because functor $\mathscr{F} \rightarrow C(\mathscr{F})$ is smooth, we can extend it to the category of vector bundles of type

$$
\xi^{\langle z\rangle}=\left\{\pi_{d}: H E^{\langle z\rangle} \oplus V_{1} E^{\langle z\rangle} \oplus \cdots \oplus V_{z} E^{\langle z\rangle} \longrightarrow E^{\langle z\rangle}\right\}
$$

Recall that by $\mathscr{F}_{F}$ we denote the typical fiber of such bundles. For $\xi^{\langle z\rangle}$, we obtain a bundle of algebras, denoted as $C\left(\xi^{\langle z\rangle}\right)$, such that $C\left(\xi^{\langle z\rangle}\right)_{u}=C\left(\mathscr{F}_{u}\right)$. Multiplication in every fiber defines a continuous map $C\left(\xi^{\langle z\rangle}\right) \times C\left(\xi^{\langle z\rangle}\right) \rightarrow C\left(\xi^{\langle z\rangle}\right)$. If $\xi^{\langle z\rangle}$ is a distinguished vector bundle on number field $k, C\left(\xi^{\langle z\rangle}\right)$-module, the d-module, on $\xi^{\langle z\rangle}$ is given by the continuous map $C\left(\xi^{\langle z\rangle}\right) \times_{E} \xi^{\langle z\rangle} \rightarrow \xi^{\langle z\rangle}$ with every fiber $\mathscr{F}_{u}$ provided with the structure of the $C\left(\mathscr{F}_{u}\right)$-module, correlated with its $k$-module structure. Because $\mathscr{F} \subset C(\mathscr{F})$, we have a 
fiber-to-fiber map $\mathscr{F}_{F} \times_{E} \xi^{\langle z\rangle} \rightarrow \xi^{\langle z\rangle}$, inducing on every fiber the map $\mathscr{F}_{u} \times_{E} \xi_{(u)}^{\langle z\rangle} \rightarrow \xi_{(u)}^{\langle z\rangle}(\mathbb{R}$ linear on the first factor and $k$-linear on the second one). Inversely, every such bilinear map defines on $\xi^{\langle z\rangle}$ the structure of the $C\left(\xi^{\langle z\rangle}\right)$-module by virtue of the universal properties of Clifford d-algebras. Equivalently, the above-mentioned bilinear map defines a morphism of v-bundles

$$
m: \xi^{\langle z\rangle} \longrightarrow \operatorname{HOM}\left(\xi^{\langle z\rangle}, \xi^{\langle z\rangle}\right)
$$

where $\operatorname{HOM}\left(\xi^{\langle z\rangle}, \xi^{\langle z\rangle}\right)$ denotes the bundles of homomorphisms when $(m(u))^{2}=G(u)$ on every point.

Vector bundles $\xi^{\langle z\rangle}$ provided with $C\left(\xi^{\langle z\rangle}\right)$-structures are objects of the category with morphisms being morphisms of dv-bundles, which induce on every point $u \in \xi^{\langle z\rangle}$ morphisms of $C\left(\mathscr{F}_{u}\right)$-modules. This is a Banach category contained in the category of finitedimensional d-vector spaces on field $k$.

We denote by $H^{s}\left(\mathscr{E}\langle z\rangle, \mathrm{GL}_{n_{E}}(\mathbb{R})\right)$, where $n_{E}=n+m_{1}+\cdots+m_{z}$, the $s$-dimensional cohomology group of the algebraic sheaf of germs of continuous maps of dv-bundle $\mathscr{E}^{\langle z\rangle}$ with group $\mathrm{GL}_{n_{E}}(\mathbb{R})$, the group of automorphisms of $\mathbb{R}^{n_{E}}$ (for the language of algebraic topology, see, e.g., [28]). We will also use the group $S L_{n_{E}}(\mathbb{R})=\left\{A \subset G L_{n_{E}}(\mathbb{R})\right.$, $\operatorname{det} A=1\}$. Here, we point out that cohomologies $H^{s}(M, G r)$ characterize the class of a principal bundle $\pi: P \rightarrow M$ on $M$ with structural group $G r$. Taking into account that we deal with bundles distinguished by an $\mathrm{N}$-connection, we introduce into consideration cohomologies $H^{s}\left(\mathscr{E}\langle z\rangle, \mathrm{GL}_{n_{E}}(\mathbb{R})\right)$ as distinguished classes (d-classes) of bundles $\mathscr{E}\langle z\rangle$ provided with a global N-connection structure.

For a real vector bundle $\xi^{\langle z\rangle}$ on compact base $\mathscr{E}^{\langle z\rangle}$, we can define the orientation on $\xi^{\langle z\rangle}$ as an element $\alpha_{d} \in H^{1}\left(\mathscr{E}\langle z\rangle, \mathrm{GL}_{n_{E}}(\mathbb{R})\right)$ whose image on map

$$
H^{1}\left(\mathscr{E}\langle z\rangle, S L_{n_{E}}(\mathbb{R})\right) \longrightarrow H^{1}\left(\mathscr{E}^{\langle z\rangle}, \mathrm{GL}_{n_{E}}(\mathbb{R})\right)
$$

is the d-class of bundle $\mathscr{E}\langle z\rangle$.

Definition 5.5. The spinor structure on $\xi^{\langle z\rangle}$ is defined as an element $\beta_{d} \in H^{1}(\mathscr{E}\langle z\rangle$, $\left.\operatorname{Spin}\left(n_{E}\right)\right)$ whose image in the composition

$$
H^{1}\left(\mathscr{E}^{\langle z\rangle}, \operatorname{Spin}\left(n_{E}\right)\right) \longrightarrow H^{1}\left(\mathscr{E}^{\langle z\rangle}, \mathrm{SO}\left(n_{E}\right)\right) \longrightarrow H^{1}\left(\mathscr{E}^{\langle z\rangle}, \mathrm{GL}_{n_{E}}(\mathbb{R})\right)
$$

is the d-class of $\mathscr{E}^{\langle z\rangle}$.

The above definition of spinor structures can be reformulated in terms of principal bundles. Let $\xi^{\langle z\rangle}$ be a real vector bundle of rank $n+m$ on a compact base $\mathscr{E}\langle z\rangle$. If there is a principal bundle $P_{d}$ with structural group $\operatorname{SO}\left(n_{E}\right)$ (or $\operatorname{Spin}\left(n_{E}\right)$ ), this bundle $\xi^{\langle z\rangle}$ can be provided with orientation (or spinor) structure. The bundle $P_{d}$ is associated with element $\alpha_{d} \in H^{1}\left(\mathscr{E}^{\langle z\rangle}, \mathrm{SO}\left(n_{\langle z\rangle}\right)\right)$ (or $\beta_{d} \in H^{1}\left(\mathscr{E}^{\langle z\rangle}, \operatorname{Spin}\left(n_{E}\right)\right)$ ).

We remark that a real bundle is oriented if and only if its first Stiefel-Whitney d-class vanishes,

$$
w_{1}\left(\xi_{d}\right) \in H^{1}\left(\xi, \frac{\mathscr{L}}{2}\right)=0
$$


where $H^{1}(\mathscr{E}\langle z\rangle, \mathscr{L} / 2)$ is the first group of Cech cohomology with coefficients in $\mathscr{L} / 2$. Considering the second Stiefel-Whitney class $w_{2}\left(\xi^{\langle z\rangle}\right) \in H^{2}(\mathscr{E}\langle z\rangle, \mathscr{L} / 2)$, it is well known that vector bundle $\xi^{\langle z\rangle}$ admits the spinor structure if and only if $w_{2}\left(\xi^{\langle z\rangle}\right)=0$. Finally, we emphasize that taking into account that base space $\mathscr{E}^{\langle z\rangle}$ is also a v-bundle, $p: E^{\langle z\rangle} \rightarrow M$, we have to make explicit calculations in order to express cohomologies $H^{s}\left(\mathscr{E}\langle z\rangle, \mathrm{GL}_{n+m}\right)$ and $H^{s}\left(\mathscr{E}^{\langle z\rangle}, \mathrm{SO}(n+m)\right)$ through cohomologies

$$
H^{s}\left(M, \mathrm{GL}_{n}\right), H^{s}\left(M, \mathrm{SO}\left(m_{1}\right)\right), \ldots, H^{s}\left(M, \mathrm{SO}\left(m_{z}\right)\right),
$$

which depend on global topological structures of spaces $M$ and $\mathscr{E}\langle z\rangle$. For general bundle and base spaces, this requires a cumbersome cohomological calculus.

5.2.2. Clifford fibration. Another way of defining the spinor structure is to use Clifford fibrations. Consider the principal bundle with the structural group $G r$ being a subgroup of orthogonal group $O(G)$, where $G$ is a quadratic nondegenerate form defined on the base (also being a bundle space) space $\mathscr{E}\langle z\rangle$. The fibration associated to principal fibration $P(\mathscr{E}\langle z\rangle, G r)$ with a typical fiber having Clifford algebra $C(G)$ is, by definition, the Clifford fibration $P C(\mathscr{E}\langle z\rangle, G r)$. We can always define a metric on the Clifford fibration if every fiber is isometric to $P C(\mathscr{E}\langle z\rangle, G)$ (this result is proved for arbitrary quadratic forms $G$ on pseudo-Riemannian bases). If, additionally, $G r \subset \mathrm{SO}(G)$, a global section can be defined on $P C(G)$.

Let $\mathscr{P}(\mathscr{E}\langle z\rangle, G r)$ be the set of principal bundles with differentiable base $\mathscr{E}\langle z\rangle$ and structural group $G r$. If $g: G r \rightarrow G r^{\prime}$ is a homomorphism of Lie groups and $P(\mathscr{E}\langle z\rangle, G r) \subset$ $\mathscr{P}\left(\mathscr{E}^{\langle z\rangle}, G r\right)$ (for simplicity in this subsection, we will denote mentioned bundles and sets of bundles as $P, P^{\prime}$, and, resp., $\left.\mathscr{P}, \mathscr{P}^{\prime}\right)$, we can always construct a principal bundle with the property that there is a homomorphism $f: P^{\prime} \rightarrow P$ of principal bundles, which can be projected to the identity map of $\mathscr{E}^{\langle z\rangle}$ and corresponds to isomorphism $g: G r \rightarrow G r^{\prime}$. If the inverse statement also holds, the bundle $P^{\prime}$ is called the extension of $P$ associated to $g$ and $f$ is called the extension homomorphism denoted as $\tilde{g}$.

Now we can define distinguished spinor structures on bundle spaces.

DEFINITION 5.6. Let $P \in \mathscr{P}\left(\mathscr{E}^{\langle z\rangle}, O(G)\right)$ be a principal bundle. A distinguished spinor structure of $P$, equivalently, a ds-structure of $\mathscr{E}\langle z\rangle$, is an extension $\widetilde{P}$ of $P$ associated to homomorphism $h: \operatorname{Pin} G \rightarrow O(G)$, where $O(G)$ is the group of orthogonal rotations, generated by metric $G$, in bundle $\mathscr{E}\langle z\rangle$.

So, if $\widetilde{P}$ is a spinor structure of the space $\mathscr{E}\langle z\rangle$, then $\widetilde{P} \in \mathscr{P}(\mathscr{E}\langle z\rangle, \operatorname{Pin} G)$.

The definition of spinor structures on varieties was given in $[16,17]$. It has been proved that a necessary and sufficient condition for a space-time to be orientable is to admit a global field of orthonormalized frames. We mention that spinor structures can also be defined on varieties modeled on Banach spaces [2]. As we have shown, similar constructions are possible for the cases when space-time has the structure of a v-bundle with an N-connection.

DEFINITION 5.7. A special distinguished spinor structure, ds-structure, of principal bundle $P=P(\mathscr{E}\langle z\rangle, \mathrm{SO}(G))$ is a principal bundle $\widetilde{P}=\widetilde{P}(\mathscr{E}\langle z\rangle$, Spin $G)$ for which a homomorphism of principal bundles $\tilde{p}: \widetilde{P} \rightarrow P$, projected onto the identity map of $\mathscr{E}\langle z\rangle$ and 
corresponding to the representation

$$
R: \operatorname{Spin} G \longrightarrow \mathrm{SO}(G)
$$

is defined.

In the case when the base space variety is oriented, there is a natural bijection between tangent spinor structures with a common base. For special ds-structures, we can define, as for any spinor structure, the concepts of spin tensors, spinor connections, and spinor covariant derivations (see $[82,83,101])$.

5.3. Almost complex spinor structures. Almost complex structures are an important characteristic of $H^{2 n}$-spaces and of osculator bundles $\operatorname{Osc}^{k=2 k_{1}}(M)$, where $k_{1}=$ $1,2, \ldots$ For simplicity in this subsection, we restrict our analysis to the case of $H^{2 n}$. spaces. We can rewrite the almost Hermitian metric $[46,47,48], H^{2 n}$-metric, in complex form [88, 89]:

$$
G=H_{a b}(z, \xi) d z^{a} \otimes d z^{b}
$$

where

$$
z^{a}=x^{a}+i y^{a}, \quad \overline{z^{a}}=x^{a}-i y^{a}, \quad H_{a b}(z, \bar{z})=\left.g_{a b}(x, y)\right|_{y=y(z, \bar{z})} ^{x=x(z, \bar{z})},
$$

and define almost complex spinor structures. For a given metric (5.16) on $H^{2 n}$-space, there is always a principal bundle $P^{U}$ with unitary structural group $U(n)$ which allows us to transform $H^{2 n}$-space into v-bundle $\xi^{U} \approx P^{U} \times_{U(n)} \mathbb{R}^{2 n}$. This statement will be proved after we introduce complex spinor structures on oriented real vector bundles [28].

We consider momentarily $k=\mathbb{C}$ and introduce into consideration (instead of the $\operatorname{group} \operatorname{Spin}(n))$ the group $\operatorname{Spin}^{c} \times_{\mathscr{E} / 2} U(1)$ being the factor group of the product $\operatorname{Spin}(n) \times$ $U(1)$ with respect to the equivalence

$$
(y, z) \sim(-y,-a), \quad y \in \operatorname{Spin}(m) .
$$

This way we define the short exact sequence

$$
1 \longrightarrow U(1) \longrightarrow \operatorname{Spin}^{c}(n) \stackrel{S^{c}}{\longrightarrow} \mathrm{SO}(n) \longrightarrow 1,
$$

where $\rho^{c}(y, a)=\rho^{c}(y)$. If $\lambda$ is oriented, real $\gamma$-bundle $\pi: E_{\lambda} \rightarrow M^{n}$ of rank $n$, with base $M^{n}$, the complex spinor structure, spin structure, on $\lambda$ is given by the principal bundle $P$ with structural group $\operatorname{Spin}^{c}(m)$ and isomorphism $\lambda \approx P \times_{\operatorname{Spin}^{c}(n)} \mathbb{R}^{n}$ (see (5.19)). For such bundles, the categorial equivalence can be defined as

$$
\epsilon^{c}: \mathscr{E}_{\mathbb{C}}^{T}\left(M^{n}\right) \longrightarrow \mathscr{E}_{\mathbb{C}}^{\lambda}\left(M^{n}\right)
$$

where $\epsilon^{\mathcal{C}}\left(E^{\mathcal{C}}\right)=P \triangle_{\operatorname{Spin}^{c}(n)} E^{\mathcal{C}}$ is the category of trivial complex bundles on $M^{n}$, $\mathscr{E}_{\mathbb{C}}^{\lambda}\left(M^{n}\right)$ is the category of complex v-bundles on $M^{n}$ with action of Clifford bundle $C(\lambda), P \triangle_{\operatorname{Spin}^{c}(n)}$, and $E^{c}$ is the factor space of the bundle product $P \times_{M} E^{c}$ with respect 
to the equivalence $(p, e) \sim\left(p \hat{\mathfrak{g}}^{-1}, \hat{\mathfrak{g}} e\right), p \in P, e \in E^{c}$, where $\hat{\mathfrak{g}} \in \operatorname{Spin}^{c}(n)$ acts on $E$ via the imbedding $\operatorname{Spin}(n) \subset C^{0, n}$ and the natural action $U(1) \subset \mathbb{C}$ on complex v-bundle $\xi^{c}$, $E^{c}=t o t \xi^{c}$, for bundle $\pi^{c}: E^{c} \rightarrow M^{n}$.

Now we return to the bundle $\xi=\mathscr{E}^{\langle 1\rangle}$. A real v-bundle (not being a spinor bundle) admits a complex spinor structure if and only if there exists a homomorphism $\sigma$ : $U(n) \rightarrow \operatorname{Spin}^{c}(2 n)$ defining a commutative diagram. The explicit construction of $\sigma$ for arbitrary $\gamma$-bundle is given in $[6,28]$. Let $\lambda$ be a complex, rank $n$, spinor bundle with

$$
\tau: \operatorname{Spin}^{c}(n) \times_{\mathscr{L} / 2} U(1) \longrightarrow U(1)
$$

the homomorphism defined by $\tau(\lambda, \delta)=\delta^{2}$. For $P_{s}$ being the principal bundle with fiber $\operatorname{Spin}^{c}(n)$, we introduce the complex linear bundle $L\left(\lambda^{c}\right)=P_{S} \times_{\operatorname{Spin}^{c}(n)} \mathbb{C}$ defined as the factor space of $P_{S} \times \mathbb{C}$ on equivalence relation

$$
(p t, z) \sim\left(p, l(t)^{-1} z\right),
$$

where $t \in \operatorname{Spin}^{c}(n)$. This linear bundle is associated to complex spinor structure on $\lambda^{c}$.

If $\lambda^{c}$ and $\lambda^{c^{\prime}}$ are complex spinor bundles, the Whitney sum $\lambda^{c} \oplus \lambda^{c^{\prime}}$ is naturally provided with the structure of the complex spinor bundle. This follows from the holomorphism

$$
\omega^{\prime}: \operatorname{Spin}^{c}(n) \times \operatorname{Spin}^{c}\left(n^{\prime}\right) \longrightarrow \operatorname{Spin}^{c}\left(n+n^{\prime}\right)
$$

given by formula $\left[(\beta, z),\left(\beta^{\prime}, z^{\prime}\right)\right] \rightarrow\left[\omega\left(\beta, \beta^{\prime}\right), z z^{\prime}\right]$, where $\omega$ is the homomorphism defining a commutative diagram of maps. Here, $z, z^{\prime} \in U(1)$. It is obvious that $L\left(\lambda^{c} \oplus \lambda^{c^{\prime}}\right)$ is isomorphic to $L\left(\lambda^{c}\right) \otimes L\left(\lambda^{c^{\prime}}\right)$.

We conclude this subsection by formulating our main result on complex spinor structures for $H^{2 n}$-spaces.

THEOREM 5.8. Let $\lambda^{c}$ be a complex spinor bundle of rank $n$ and $H^{2 n}$-space considered as a real vector bundle $\lambda^{c} \oplus \lambda^{c^{\prime}}$ provided with almost complex structure $J_{\beta}^{\alpha}$; multiplication on $i$ is given by $\left(\begin{array}{cc}0 & -\delta_{j}^{i} \\ \delta_{j}^{i} & 0\end{array}\right)$. Then, there is a diagram of maps which is commutative up to isomorphisms $\epsilon^{c}$ and $\tilde{\epsilon}^{c}$ defined as in (5.20), He is a functor $E^{c} \rightarrow E^{c} \otimes L\left(\lambda^{c}\right), \mathscr{E}_{\mathbb{C}}^{0,2 n}\left(M^{n}\right)$ is defined by functor $\mathscr{E}_{\mathbb{C}}\left(M^{n}\right) \rightarrow \mathscr{E}_{\mathbb{C}}^{0,2 n}\left(M^{n}\right)$ given as correspondence $E^{c} \rightarrow \Lambda\left(\mathbb{C}^{n}\right) \otimes E^{c}$ (which is a categorial equivalence), $\Lambda\left(\mathbb{C}^{n}\right)$ is the exterior algebra on $\mathbb{C}^{n}$, and $W$ is the real bundle $\lambda^{c} \oplus \lambda^{c^{\prime}}$ provided with complex structure.

Proof. See [88, 89, 92, 93, 106].

Now consider bundle $P \times \operatorname{Spin}^{c}(n) \operatorname{Spin}^{c}(2 n)$ as the principal $\operatorname{Spin}^{c}(2 n)$-bundle, associated to $M \oplus M$ being the factor space of the product $P \times \operatorname{Spin}^{c}(2 n)$ on the equivalence relation $(p, t, h) \sim\left(p, \mu(t)^{-1} h\right)$. In this case, the categorial equivalence (5.20) can be rewritten as

$$
\epsilon^{c}\left(E^{c}\right)=P \times_{\operatorname{Spin}^{c}(n)} \operatorname{Spin}^{c}(2 n) \Delta_{\operatorname{Spin}^{c}(2 n)} E^{c}
$$

and seen as a factor space of $P \times \operatorname{Spin}^{c}(2 n) \times_{M} E^{c}$ on equivalence relations

$$
(p t, h, e) \sim\left(p, \mu(t)^{-1} h, e\right), \quad\left(p, h_{1}, h_{2}, e\right) \sim\left(p, h_{1}, h_{2}^{-1} e\right)
$$


(projections of elements $p$ and $e$ coincide on base $M$ ). Every element of $\epsilon^{c}\left(E^{c}\right)$ can be represented as $P \Delta_{\operatorname{Spin}^{c}(n)} E^{c}$, that is, as a factor space $P \Delta E^{c}$ on equivalence relation $(p t, e) \sim\left(p, \mu^{c}(t), e\right)$, when $t \in \operatorname{Spin}^{c}(n)$. The complex line bundle $L\left(\lambda^{c}\right)$ can be interpreted as the factor space of $P \times_{\operatorname{Spin}^{c}(n)} \mathbb{C}$ on equivalence relation $(p t, \delta) \sim\left(p, r(t)^{-1} \delta\right)$.

Putting $(p, e) \otimes(p, \delta)(p, \delta e)$, we introduce morphism

$$
\epsilon^{c}(E) \times L\left(\lambda^{c}\right) \longrightarrow \epsilon^{c}\left(\lambda^{c}\right)
$$

with properties

$$
\begin{gathered}
(p t, e) \otimes(p t, \delta) \longrightarrow(p t, \delta e)=\left(p, \mu^{c}(t)^{-1} \delta e\right) \\
\left(p, \mu^{c}(t)^{-1} e\right) \otimes\left(p, l(t)^{-1} e\right) \longrightarrow\left(p, \mu^{c}(t) r(t)^{-1} \delta e\right)
\end{gathered}
$$

pointing to the fact that we have defined the isomorphism correctly and that it is an isomorphism on every fiber.

6. Spinors and N-connection geometry. The purpose of this section is to show how a corresponding abstract spinor technique entailing notational and calculational advantages can be developed for arbitrary splits of dimensions of a d-vector space $\mathscr{F}=h \mathscr{F} \oplus v_{1} \mathscr{F}_{\mathscr{F}} \oplus \oplus v_{z} \mathscr{F}$, where $\operatorname{dim} h \mathscr{F}=n$ and $\operatorname{dim} v_{p} \mathscr{F}=m_{p}$. For convenience, we will also present some necessary coordinate expressions.

6.1. d-spinor techniques. The problem of a rigorous definition of spinors on locally anisotropic spaces (d-spinors) was posed and solved [82, 83, 88, 89] in the framework of the formalism of Clifford and spinor structures on v-bundles provided with compatible nonlinear and distinguished connections and metric. We introduced d-spinors as corresponding objects of the Clifford d-algebra $\mathbb{C}(\mathscr{F}, G)$, defined for a d-vector space $\mathscr{F}_{\mathscr{F}}$ in a standard manner (see, e.g., [28]) and proved that operations with $\mathbb{C}(\mathscr{F}, G)$ can be reduced to calculations for $\mathbb{C}(h \mathscr{F}, g), \mathbb{C}\left(v_{1} \mathscr{F}, h_{1}\right), \ldots, \mathbb{C}\left(v_{z} \mathscr{F}, h_{z}\right)$, which are usual Clifford algebras of respective dimensions $2^{n}, 2^{m_{1}}, \ldots, 2^{m_{z}}$ (if it is necessary, we can use quadratic forms $g$ and $h_{p}$ correspondingly induced on $h \mathscr{F}$ and $v_{p} \mathscr{F}_{F}$ by a metric G (3.28)). Considering the orthogonal subgroup $O(\mathbf{G}) \subset \mathrm{GL}(\mathbf{G})$ defined by a metric $\mathbf{G}$, we can define the d-spinor norm and parametrize d-spinors by ordered pairs of elements of Clifford algebras $\mathbb{C}(h \mathscr{F}, g)$ and $\mathbb{C}\left(v_{p} \mathscr{F}, h_{p}\right), p=1,2, \ldots, z$. We emphasize that the splitting of a Clifford d-algebra associated to a dv-bundle $\mathscr{E}^{\langle z\rangle}$ is a straightforward consequence of the global decomposition defining an N-connection structure in $\mathscr{E}\langle z\rangle$.

In this subsection, we will omit detailed proofs which in most cases are mechanical but rather tedious. We can apply the methods developed in $[7,9,26,27,31,54$, $61,62,63]$ in a straightforward manner on $\mathrm{h}$ - and v-subbundles in order to verify the correctness of affirmations.

6.1.1. Clifford d-algebra, $d$-spinors, and d-twistors. In order to relate the succeeding constructions with Clifford d-algebras $[88,89]$ we consider a la-frame decomposition of the metric (3.28):

$$
G_{\langle\alpha\rangle\langle\beta\rangle}(u)=l_{\langle\alpha\rangle}^{\langle\hat{\alpha}\rangle}(u) l_{\langle\beta\rangle}^{\langle\hat{\beta}\rangle}(u) G_{\langle\hat{\alpha}\rangle\langle\hat{\beta}\rangle},
$$


where the frame d-vectors and constant metric matrices are distinguished as

$$
\begin{aligned}
l_{\langle\alpha\rangle}^{\langle\hat{\alpha}\rangle}(u) & =\left(\begin{array}{cccc}
l_{j}^{\hat{j}}(u) & 0 & \cdots & 0 \\
0 & l_{a_{1}}^{\hat{a}_{1}(u)} & \cdots & 0 \\
\vdots & \vdots & \vdots & \vdots \\
0 & 0 & \cdots & l_{a_{z}}^{\hat{a}_{z}}(u)
\end{array}\right), \\
G_{\langle\hat{\alpha}\rangle\langle\hat{\beta}\rangle} & =\left(\begin{array}{cccc}
g_{\hat{i} \hat{j}} & 0 & \cdots & 0 \\
0 & h_{\hat{a}_{1} \hat{b}_{1}} & \cdots & 0 \\
\vdots & \vdots & \vdots & \vdots \\
0 & 0 & 0 & h_{\hat{a}_{z} \hat{b}_{z}}
\end{array}\right),
\end{aligned}
$$

$g_{\hat{i} \hat{j}}$ and $h_{\hat{a}_{1} \hat{b}_{1}}, \ldots, h_{\hat{a}_{z} \hat{b}_{z}}$ are diagonal matrices with $g_{\hat{i} \hat{i}}=h_{\hat{a}_{1} \hat{a}_{1}}=\cdots=h_{\hat{a}_{z} \hat{b}_{z}}= \pm 1$.

To generate Clifford d-algebras, we start with matrix equations

$$
\sigma_{\langle\hat{\alpha}\rangle} \sigma_{\langle\hat{\beta}\rangle}+\sigma_{\langle\hat{\beta}\rangle} \sigma_{\langle\hat{\alpha}\rangle}=-G_{\langle\hat{\alpha}\rangle\langle\hat{\beta}\rangle} I,
$$

where $I$ is the identity matrix, matrices $\sigma_{\langle\hat{\alpha}\rangle}$ ( $\sigma$-objects) act on a d-vector space $\mathscr{F}=$ $h \mathscr{F} \oplus v_{1} \mathscr{F} \oplus \cdots \oplus v_{z} \mathscr{F}$, and their components are distinguished as

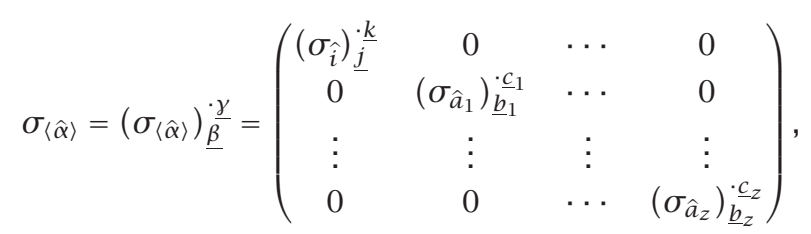

indices $\underline{\beta}, \underline{\gamma}, \ldots$ refer to spin spaces of type $\mathscr{S}=S_{(h)} \oplus S_{\left(v_{1}\right)} \oplus \cdots \oplus S_{\left(v_{z}\right)}$, and underlined Latin indices $j, \underline{k}, \ldots$ and $\underline{b}_{1}, \underline{c}_{1}, \ldots, \underline{b}_{z}, \underline{c}_{z} \ldots$ refer, respectively, to h-spin space $\mathscr{S}_{(h)}$ and $\mathrm{v}_{p}$-spin space $\mathscr{S}_{\left(v_{p}\right)}(p=1,2, \ldots, z)$, which are correspondingly associated to h- and $v_{p}$-decompositions of a dv-bundle $\mathscr{E}\langle z\rangle$. The irreducible algebra of matrices $\sigma_{\langle\hat{\alpha}\rangle}$ of minimal dimension $N \times N$, where $N=N_{(n)}+N_{\left(m_{1}\right)}+\cdots+N_{\left(m_{z}\right)}$, $\operatorname{dim} \mathscr{Y}_{(h)}=N_{(n)}$, and $\operatorname{dim} \mathscr{Y}_{\left(v_{p}\right)}=N_{\left(m_{p}\right)}$, has the dimensions

$$
N_{(n)}=\left\{\begin{array}{ll}
2^{(n-1) / 2}, & n=2 k+1, \\
2^{n / 2}, & n=2 k,
\end{array} \quad N_{\left(m_{p}\right)}=\left\{\begin{array}{ll}
2^{\left(m_{p}-1\right) / 2}, & m_{p}=2 k_{p}+1 \\
2^{m_{p}}, & m_{p}=2 k_{p}
\end{array},\right.\right.
$$

where $k, k_{p}=1,2, \ldots$.

The Clifford d-algebra is generated by sums on $n+1$ elements of the form

$$
A_{1} I+B^{\hat{i}} \sigma_{\hat{i}}+C^{\hat{i} \hat{j}} \sigma_{\hat{i} \hat{j}}+D^{\hat{i} \hat{j} \hat{k}} \sigma_{\hat{i} \hat{j} \hat{k}}+\cdots
$$

and sums of $m_{p}+1$ elements of the form

$$
A_{2(p)} I+B^{\hat{a}_{p}} \sigma_{\hat{a}_{p}}+C^{\hat{a}_{p} \hat{b}_{p}} \sigma_{\hat{a}_{p} \hat{b}_{p}}+D^{\hat{a}_{p} \hat{b}_{p} \hat{c}_{p}} \sigma_{\hat{a}_{p} \hat{b}_{p} \hat{c}_{p}}+\cdots
$$

with antisymmetric coefficients $C^{\hat{i} \hat{j}}=C^{[\hat{i} \hat{j}]}, C^{\hat{a}_{p} \hat{b}_{p}}=C^{\left[\hat{a}_{p} \hat{b}_{p}\right]}, D^{\hat{i} \hat{j} \hat{k}}=D^{[\hat{i} \hat{j} \hat{k}]}, D^{\hat{a}_{p} \hat{b}_{p} \hat{c}_{p}}=$ $D^{\left[\hat{a}_{p} \hat{b}_{p} \hat{c}_{p}\right]}, \ldots$ and matrices $\sigma_{\hat{i} \hat{j}}=\sigma_{[\hat{i}} \sigma_{\hat{j}]}, \sigma_{\hat{a}_{p} \hat{b}_{p}}=\sigma_{\left[\hat{a}_{p}\right.} \sigma_{\left.\hat{b}_{p}\right]}, \sigma_{\hat{i} \hat{j} \hat{k}}=\sigma_{[\hat{i}} \sigma_{\hat{j}} \sigma_{\hat{k}]}, \ldots$ Really, 
we have $2^{n+1}$ coefficients $\left(A_{1}, C^{\hat{i} \hat{j}}, D^{\hat{i} \hat{j} \hat{k}}, \ldots\right)$ and $2^{m_{p}+1}$ coefficients $\left(A_{2(p)}, C^{\hat{a}_{p} \hat{b}_{p}}, D^{\hat{a}_{p} \hat{b}_{p} \hat{c}_{p}}\right.$, ...) of the Clifford algebra on $\mathscr{F}$.

For simplicity, we will present the necessary geometric constructions only for $\mathrm{h}$-spin spaces $\mathscr{S}_{(h)}$ of dimension $N_{(n)}$. Considerations for a v-spin space $\mathscr{S}_{(v)}$ are similar but with proper characteristics for a dimension $N_{(m)}$.

In order to define the scalar (spinor) product on $\mathscr{S}_{(h)}$, we introduce into consideration the following finite sum (because of a finite number of elements $\sigma_{[\hat{i} \hat{j} \ldots \hat{k}]}$ ):

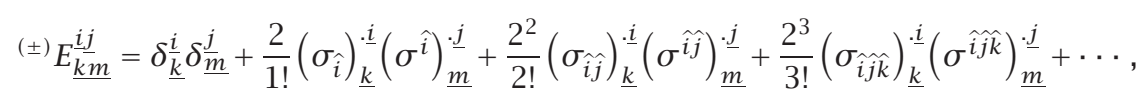

which can be factorized as

$$
\begin{aligned}
& { }^{( \pm)} E_{\underline{k} \underline{\underline{j}}}^{\underline{i} \underline{j}}=N_{(n)}{ }^{( \pm)} \epsilon_{\underline{k} \underline{m}}{ }^{( \pm)} \epsilon^{\underline{i} \underline{j}} \text { for } n=2 k,
\end{aligned}
$$

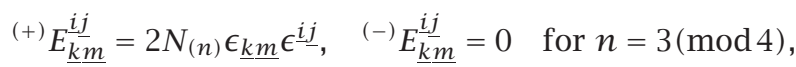

$$
\begin{aligned}
& { }^{(+)} E_{\underline{k} \underline{\underline{m}}}^{\underline{i j}}=0, \quad{ }^{(-)} E_{\underline{\underline{k}} \underline{\underline{j}}}^{\underline{\underline{j}}}=2 N_{(n)} \epsilon_{\underline{k} \underline{m}} \epsilon^{\underline{\underline{i}} \underline{j}} \text { for } n=1(\bmod 4) \text {. }
\end{aligned}
$$

Antisymmetry of $\sigma_{\widehat{i} \hat{j} \hat{k} \ldots . . .}$ and the construction of the objects (6.6), (6.8), (6.9), and (6.10) define the properties of $\epsilon$-objects ${ }^{( \pm)} \epsilon_{\underline{k} \underline{\underline{m}}}$ and $\epsilon_{\underline{k} \underline{\underline{m}}}$ which have an eight-fold periodicity on $n$ (see details in [61, 62, 63] and, with respect to locally anisotropic spaces, [88, 89]).

For even values of $n$, it is possible the decomposition of every h-spin space $\mathscr{S}_{(h)}$ into irreducible h-spin spaces $\mathbf{S}_{(h)}$ and $\mathbf{S}_{(h)}^{\prime}$ (one considers splitting of h-indices, e.g., $\underline{l}=L \oplus L^{\prime}, \underline{m}=M \oplus M^{\prime}, \ldots$; for $\mathrm{v}_{p}$-indices, we will write $\left.\underline{a}_{p}=A_{p} \oplus A_{p}^{\prime}, \underline{b}_{p}=B_{p} \oplus B_{p}^{\prime}, \ldots\right)$ and one defines new $\epsilon$-objects

$$
\epsilon^{\underline{\underline{l}} \underline{\underline{m}}}=\frac{1}{2}\left({ }^{(+)} \epsilon^{\underline{\underline{l m}}}+{ }^{(-)} \epsilon^{\underline{\underline{l m}}}\right), \quad \tilde{\epsilon} \epsilon^{\underline{\underline{l}}}=\frac{1}{2}\left({ }^{(+)} \epsilon^{\underline{\underline{\underline{m}}}}{ }_{-}^{(-)} \epsilon^{\underline{\underline{l m}}}\right) .
$$

We will omit similar formulas for $\epsilon$-objects with lower indices.

In general, the spinor $\epsilon$-objects should be defined for every shell of anisotropy, where instead of dimension $n$, we will consider the dimensions $m_{p}, 1 \leq p \leq z$, of shells.

We define a d-spinor space $\mathscr{S}_{\left(n, m_{1}\right)}$ as a direct sum of horizontal and vertical spinor spaces, for instance,

$$
\begin{gathered}
\mathscr{S}_{\left(8 k, 8 k^{\prime}\right)=} \mathbf{S}_{\circ} \oplus \mathbf{S}_{\circ}^{\prime} \oplus \mathbf{S}_{\left.\right|_{\circ} \oplus \mathbf{S}_{\mid \circ}^{\prime},}, \mathscr{S}_{\left(8 k, 8 k^{\prime}+1\right)}=\mathbf{S}_{\circ} \oplus \mathbf{S}_{\circ}^{\prime} \oplus \mathscr{Y}_{\left.\right|_{\circ}}^{(-)}, \ldots, \\
\mathscr{S}_{\left(8 k+4,8 k^{\prime}+5\right)}=\mathbf{S}_{\triangle} \oplus \mathbf{S}_{\triangle}^{\prime} \oplus \mathscr{S}_{\mid \triangle}^{(-)}, \ldots
\end{gathered}
$$

The scalar product on a $\mathscr{S}_{\left(n, m_{1}\right)}$ is induced by $\epsilon$-objects (corresponding to fixed values of $n$ and $m_{1}$ ) considered for $\mathrm{h}$ - and $\mathrm{v}_{1}$-components. We present also an example for $\mathscr{S}_{\left(n, m_{1}+\cdots+m_{z}\right)}$ :

$$
\begin{aligned}
& \mathscr{S}_{\left(8 k+4,8 k_{(1)}+5, \ldots, 8 k_{(p)}+4, \ldots, 8 k_{(z)}\right)} \\
& \quad=\mathbf{S}_{\triangle} \oplus \mathbf{S}_{\triangle}^{\prime} \oplus \mathscr{Y}_{(1) \Delta}^{(-)} \oplus \cdots \oplus \mathbf{S}_{\mid(p) \Delta} \oplus \mathbf{S}_{\mid(p) \Delta}^{\prime} \oplus \cdots \oplus \mathbf{S}_{\mid(z) \circ} \oplus \mathbf{S}_{\mid(z) \circ}^{\prime} .
\end{aligned}
$$


Having introduced d-spinors for dimensions $\left(n, m_{1}+\cdots+m_{z}\right)$, we can write out the generalization for ha-spaces of twistor equations $[61,62,63]$ by using the distinguished $\sigma$-objects (6.4):

$$
\left(\sigma_{\langle\hat{\alpha}\rangle}\right)_{|\underline{\underline{\beta}}|}^{\cdot \cdot \underline{\underline{\gamma}}} \frac{\delta \omega \underline{\underline{\beta}}}{\delta u^{\langle\hat{\beta}\rangle}}=\frac{1}{n+m_{1}+\cdots+m_{z}} G_{\langle\hat{\alpha}\rangle\langle\hat{\beta}\rangle}\left(\sigma^{\hat{\epsilon}}\right)_{\underline{\underline{\beta}}}^{\cdot{ }_{\underline{\beta}}^{\underline{\underline{\gamma}}}} \frac{\delta \omega \underline{\underline{\beta}}}{\delta u^{\hat{\epsilon}}},
$$

where $|\underline{\beta}|$ denotes that we do not consider symmetrization on this index. The general solution of (6.14) on the d-vector space $\mathscr{F}$ looks like

$$
\omega^{\underline{\beta}}=\Omega^{\underline{\beta}}+u^{\langle\hat{\alpha}\rangle}\left(\sigma_{\langle\hat{\alpha}\rangle}\right)_{\underline{\epsilon}}^{\cdot \underline{\underline{\beta}}} \Pi^{\underline{\epsilon}},
$$

where $\Omega^{\underline{\beta}}$ and $\Pi \underline{\underline{\epsilon}}$ are constant d-spinors. For fixed values of dimensions $n$ and $m=$ $m_{1}+\cdots+m_{z}$, we must analyze the reduced and irreducible components of $\mathrm{h}-$ and $\mathrm{v}_{p^{-}}$ parts of (6.14) and their solutions (6.15) in order to find the symmetry properties of a dtwistor $\mathbf{Z}^{\alpha}$ defined as a pair of d-spinors $\mathbf{Z}^{\alpha}=\left(\omega \underline{\alpha}, \pi_{\underline{\beta}}^{\prime}\right)$, where $\pi_{\underline{\beta}^{\prime}}=\pi_{{\underline{\beta^{\prime}}}^{\prime}}^{(0)} \in \tilde{\mathscr{Y}}_{\left(n, m_{1}, \ldots, m_{z}\right)}$ is a constant dual d-spinor. The problem of the definition of spinors and twistors on haspaces was firstly considered in [101] (see also [86]) in connection with the possibility to extend (6.15) and their solutions (6.16), by using nearly autoparallel maps on curved, locally isotropic or anisotropic, spaces. We note that the definition of twistors has been extended to higher-order anisotropic spaces with trivial $\mathrm{N}$ - and d-connections.

6.1.2. Mutual transforms of d-tensors and d-spinors. The spinor algebra for spaces of higher dimensions cannot be considered as a real alternative to the tensor algebra as for locally isotropic spaces of dimensions $n=3,4[61,62,63]$. The same holds true for ha-spaces and we emphasize that it is not quite convenient to perform a spinor calculus for dimensions $n, m \gg 4$. The concept of spinors is important for every type of spaces. We can deeply understand the fundamental properties of geometrical objects on haspaces, and we will consider in this subsection some questions concerning transforms of d-tensor objects into d-spinor ones.

6.1.3. Transformation of d-tensors into d-spinors. In order to pass from d-tensors to d-spinors, we must use $\sigma$-objects (6.4) written in reduced or irreduced form (depending on fixed values of dimensions $n$ and $m$ ):

$$
\left(\sigma_{\langle\hat{\alpha}\rangle}\right)_{\underline{\beta}}^{\cdot \underline{\gamma}},\left(\sigma^{\langle\hat{\alpha}\rangle}\right)^{\underline{\beta} \underline{\gamma}},\left(\sigma^{\langle\hat{\alpha}\rangle}\right)_{\underline{\beta} \underline{\gamma}}, \ldots,\left(\sigma_{\langle\hat{a}\rangle}\right)^{\underline{b} \underline{c}}, \ldots,\left(\sigma_{\hat{i}}\right)_{\underline{j} \underline{k}}, \ldots,\left(\sigma_{\langle\hat{a}\rangle}\right)^{A A^{\prime}}, \ldots,\left(\sigma^{\hat{i}}\right)_{I I^{\prime}}, \ldots
$$

It is obvious that contracting with corresponding $\sigma$-objects (6.16), we can introduce instead of d-tensors indices the d-spinor ones, for instance,

$$
\omega^{\underline{\beta} \underline{\gamma}}=\left(\sigma^{\langle\hat{\alpha}\rangle}\right)^{\underline{\underline{\beta}} \underline{\gamma}} \omega_{\langle\hat{\alpha}\rangle}, \omega_{A B^{\prime}}=\left(\sigma^{\langle\hat{a}\rangle}\right)_{A B^{\prime}} \omega_{\langle\hat{a}\rangle}, \ldots, \zeta_{\cdot \underline{j}}^{\underline{i}}=\left(\sigma^{\hat{k}}\right)_{\cdot \underline{j}}^{\underline{i}} \zeta_{\hat{k}}, \ldots
$$

For d-tensors containing groups of antisymmetric indices, there is a more simple procedure of their transforming into d-spinors because the objects

$$
\left(\sigma_{\hat{\alpha} \hat{\beta} \cdots \hat{\gamma}}\right)^{\underline{\delta} \underline{v}},\left(\sigma^{\hat{a} \hat{b} \cdots \hat{c}}\right)^{\underline{d} \underline{e}}, \ldots,\left(\sigma^{\hat{i} \hat{j} \cdots \hat{k}}\right)_{I I^{\prime}}, \ldots
$$


can be used for sets of such indices into pairs of d-spinor indices. We enumerate some properties of $\sigma$-objects of type (6.18) (for simplicity, we consider only h-components having $q$ indices $\hat{i}, \hat{j}, \hat{k}, \ldots$ taking values from 1 to $n$; the properties of $\mathrm{v}_{p}$-components can be written in a similar manner with respect to indices $\hat{a}_{p}, \hat{b}_{p}, \hat{c}_{p}, \ldots$ taking values from 1 to $m$ ):

$$
\left(\sigma_{\hat{i} \cdots \hat{j}}\right)^{\underline{k} \underline{l}} \text { is } \begin{cases}\text { symmetric on } \underline{k}, \underline{l} & \text { for } n-2 q \equiv 1,7(\bmod 8), \\ \text { antisymmetric on } \underline{k}, \underline{l} & \text { for } n-2 q \equiv 3,5(\bmod 8),\end{cases}
$$

for odd values of $n$, and an object

$$
\begin{aligned}
& \left(\sigma_{\hat{i} \cdots \hat{j}}\right)^{I J}\left(\left(\sigma_{\hat{i} \cdots \hat{j}}\right)^{I^{\prime} J^{\prime}}\right) \\
& \text { is } \begin{cases}\text { symmetric on } I, J\left(I^{\prime}, J^{\prime}\right) & \text { for } n-2 q \equiv 0(\bmod 8), \\
\text { antisymmetric on } I, J\left(I^{\prime}, J^{\prime}\right) & \text { for } n-2 q \equiv 4(\bmod 8),\end{cases}
\end{aligned}
$$

or

$$
\left(\sigma_{\hat{i} \cdots \hat{j}}\right)^{I J^{\prime}}= \pm\left(\sigma_{\hat{i} \cdots \hat{j}}\right)^{J^{\prime} I}\left\{\begin{array}{l}
n+2 q \equiv 6(\bmod 8) \\
n+2 q \equiv 2(\bmod 8)
\end{array}\right.
$$

with vanishing of the rest of reduced components of the d-tensor $\left(\sigma_{\hat{i} \cdots \hat{j}}\right)^{\underline{k} \underline{l}}$ with prime/ unprime sets of indices.

6.1.4. Fundamental d-spinors. We can transform every d-spinor $\xi^{\underline{\alpha}}=\left(\xi^{\underline{i}}, \xi^{\underline{a_{1}}}, \ldots\right.$, $\xi^{\underline{a_{z}}}$ ) into a corresponding d-tensor. For simplicity, we consider this construction only for an h-component $\xi^{\underline{i}}$ on an h-space being of dimension $n$. The values

$$
\xi^{\underline{\alpha}} \underline{\underline{\beta}}\left(\sigma^{\hat{i} \cdots \hat{j}}\right)_{\underline{\alpha} \underline{\beta}} \quad(n \text { is odd })
$$

or

$$
\xi^{I} \xi^{J}\left(\sigma^{\hat{i} \cdots \hat{j}}\right)_{I J} \quad\left(\text { or } \xi^{I^{\prime}} \xi^{J^{\prime}}\left(\sigma^{\hat{i} \cdots \hat{j}}\right)_{I^{\prime} J^{\prime}}\right) \quad(n \text { is even })
$$

with a different number of indices $\hat{i} \cdots \hat{j}$, taken together, define the h-spinor $\xi^{\underline{i}}$ to an accuracy to the sign. We emphasize that it is necessary to choose only those hcomponents of d-tensors (6.22) (or (6.23)) which are symmetric on pairs of indices $\underline{\alpha} \underline{\beta}$ (or $I J$ (or $\left.I^{\prime} J^{\prime}\right)$ ) and the number $q$ of indices $\hat{i} \cdot \hat{j}$ satisfies the condition (as a respective consequence of the properties (6.19) and/or (6.20), (6.21))

$$
n-2 q \equiv 0,1,7(\bmod 8)
$$

Of special interest is the case when

$$
q=\frac{1}{2}(n \pm 1) \quad(n \text { is odd })
$$


or

$$
q=\frac{1}{2} n \quad(n \text { is even })
$$

If all expressions (6.22) and/or (6.23) are zero for all values of $q$ with the exception of one or two ones defined by the conditions (6.24), (6.25) (or (6.26)), the value $\xi^{\hat{i}}$ (or $\xi^{I}$ $\left.\left(\xi^{I^{\prime}}\right)\right)$ is called a fundamental h-spinor. Defining in a similar manner the fundamental $\mathrm{v}$-spinors, we can introduce fundamental d-spinors as pairs of fundamental h- and vspinors. Here we remark that an $\mathrm{h}\left(\mathrm{v}_{p}\right)$-spinor $\xi^{\hat{i}}\left(\xi^{\hat{a}_{p}}\right)$ (we can also consider reduced components) is always a fundamental one for $n(m)<7$, which is a consequence of (6.26).

6.2. Differential geometry of ha-spinors. This subsection is devoted to the differential geometry of d-spinors in higher-order anisotropic spaces. We will use denotations of type

$$
\begin{aligned}
& v^{\langle\alpha\rangle}=\left(v^{i}, v^{\langle a\rangle}\right) \in \sigma^{\langle\alpha\rangle}=\left(\sigma^{i}, \sigma^{\langle a\rangle}\right), \\
& \zeta^{\underline{\alpha}_{p}}=\left(\zeta^{\underline{\underline{i}} p}, \zeta^{\underline{a_{p}}}\right) \in \sigma^{\alpha_{p}}=\left(\sigma^{i_{p}}, \sigma^{a_{p}}\right)
\end{aligned}
$$

for, respectively, elements of modules of d-vector and irreduced d-spinor fields (see details in $[88,89]$ ). We will interpret d-tensors and d-spinor tensors (irreduced or reduced) as elements of corresponding $\sigma$-modules, for instance,

$$
q_{\langle\beta\rangle}^{\langle\alpha\rangle} \in \sigma^{\langle\alpha\rangle} /\left[-0_{\langle\beta\rangle}, \psi_{\underline{\beta}_{p}}^{\underline{\alpha}_{p} \underline{\gamma}_{p}} \in \sigma_{\underline{\beta}_{p}}^{\underline{\alpha}_{p} \underline{\gamma}_{p}}, \xi_{J p K_{p}^{\prime} N_{p}^{\prime}}^{I_{p} I_{p}^{\prime}} \in \sigma_{J_{p} K_{p}^{\prime} N_{p}^{\prime}}^{I_{p} I_{p}^{\prime}}, \ldots\right] .
$$

We can establish a correspondence between the higher-order anisotropic adapted to the N-connection metric $g_{\alpha \beta}$ (3.28) and d-spinor metric $\epsilon_{\alpha \beta}$ ( $\epsilon$-objects for both h- and $\mathrm{v}_{p}$-subspaces of $\mathscr{E}\langle z\rangle$ of a ha-space $\mathscr{E}^{\langle z\rangle}$ by using the relation

$$
\begin{aligned}
g_{\langle\alpha\rangle\langle\beta\rangle}= & -\frac{1}{N(n)+N\left(m_{1}\right)+\cdots+N\left(m_{z}\right)} \\
& \times\left(\left(\sigma_{\langle\alpha\rangle}(u)\right)^{\underline{\alpha} \underline{\beta}}\left(\sigma_{\langle\beta\rangle}(u)\right)^{\underline{\delta} \underline{\gamma}}\right) \epsilon_{\underline{\alpha} \underline{\underline{\gamma}}} \epsilon_{\underline{\beta} \underline{\delta}},
\end{aligned}
$$

where

$$
\left(\sigma_{\langle\alpha\rangle}(u)\right)^{\underline{\underline{\nu}}}=l_{\langle\alpha\rangle}^{\langle\hat{\alpha}\rangle}(u)\left(\sigma_{\langle\hat{\alpha}\rangle}\right)^{\langle\underline{\underline{v}\rangle\langle\underline{\gamma}\rangle}},
$$

which is a consequence of formulas (6.3), (6.4), (6.6), (6.8), (6.9), (6.10), and (6.11). In brief, we can write (6.29) as

$$
\mathcal{g}_{\langle\alpha\rangle\langle\beta\rangle}=\epsilon_{\underline{\alpha}_{1} \underline{\alpha}_{2}} \epsilon_{\underline{\beta}_{1}} \underline{\beta}_{2}
$$

if the $\sigma$-objects are considered as a fixed structure, whereas $\epsilon$-objects are treated as carrying the metric "dynamics" on higher-order anisotropic space. This variant is used, for instance, in the so-called 2-spinor geometry $[61,62,63]$ and should be preferred if we have to make explicit the algebraic symmetry properties of d-spinor objects by using 
metric decomposition (6.31). An alternative way is to consider as fixed the algebraic structure of $\epsilon$-objects and to use variable components of $\sigma$-objects of type (6.30) for developing a variational d-spinor approach to gravitational and matter field interactions on ha-spaces (the spinor Ashtekar variables [5] are introduced in this manner).

We note that a d-spinor metric

$$
\epsilon_{\underline{\underline{\underline{T}}} \underline{\underline{\tau}}}=\left(\begin{array}{cccc}
\epsilon_{\underline{\underline{j}} \underline{j}} & 0 & \cdots & 0 \\
0 & \epsilon_{\underline{a}_{1} \underline{b}_{1}} & \cdots & 0 \\
\vdots & \vdots & \vdots & \vdots \\
0 & 0 & \cdots & \epsilon_{\underline{a}_{z} \underline{b}_{z}}
\end{array}\right)
$$

on the d-spinor space $\mathscr{S}=\left(\mathscr{S}_{(h)}, \mathscr{Y}_{\left(v_{1}\right)}, \ldots, \mathscr{Y}_{\left(v_{z}\right)}\right)$ can have symmetric or antisymmetric $\mathrm{h}$ $\left(\mathrm{v}_{p}\right)$-components $\epsilon_{\underline{i j}}\left(\epsilon_{\underline{a}_{p} \underline{b}_{p}}\right)$, see $\epsilon$-objects. For simplicity, in order to avoid cumbersome calculations connected with eight-fold periodicity on dimensions $n$ and $m_{p}$ of a haspace $\mathscr{E}^{\langle z\rangle}$, we will develop a general d-spinor formalism only by using irreduced spinor spaces $\mathscr{Y}_{(h)}$ and $\mathscr{S}_{\left(v_{p}\right)}$.

6.2.1. d-covariant derivation on ha-spaces. Let $\mathscr{E}\langle z\rangle$ be a ha-space. We define the action on a d-spinor of a d-covariant operator

$$
\begin{aligned}
\nabla\langle\alpha\rangle= & \left(\nabla_{i}, \nabla_{\langle a\rangle}\right)=\left(\sigma_{\langle\alpha\rangle}\right)^{\underline{\alpha}_{1} \underline{\alpha}_{2}} \nabla_{\underline{\alpha}_{1} \underline{\alpha}_{2}} \\
= & \left(\left(\sigma_{i}\right)^{\underline{\underline{i}}_{1} \underline{\underline{i}}_{2}} \nabla_{\underline{\underline{i}}_{1} \underline{\underline{1}}_{2}},\left(\sigma_{\langle a\rangle}\right)^{\underline{a}_{1} \underline{a}_{2}} \nabla_{\underline{a}_{1} \underline{a}_{2}}\right) \\
= & \left(\left(\sigma_{i}\right)^{{ }_{1} \underline{\underline{i}}_{2}} \nabla_{\underline{\underline{i}}_{1} \underline{\underline{i}}_{2}},\left(\sigma_{a_{1}}\right)^{\underline{a}_{1} \underline{a}_{2}} \nabla_{(1)} \underline{a}_{1} \underline{a}_{2}, \ldots,\right. \\
& \left.\left(\sigma_{a_{p}}\right)^{\underline{a}_{1} \underline{a}_{2}} \nabla_{(p)} \underline{a}_{1} \underline{a}_{2}, \ldots,\left(\sigma_{a_{z}}\right)^{\underline{a}_{1} \underline{a}_{2}} \nabla_{(z) \underline{a}_{1} \underline{a}_{2}}\right)
\end{aligned}
$$

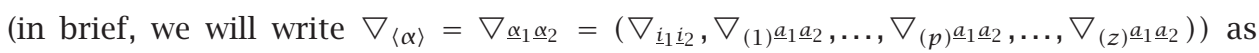
maps

$$
\begin{aligned}
& \nabla_{\underline{\alpha}_{1} \underline{\alpha}_{2}}: \sigma^{\underline{\beta}} \longrightarrow \sigma_{\langle\alpha\rangle}^{\underline{\beta}}=\sigma_{\underline{\alpha}_{1} \underline{\alpha}_{2}}^{\underline{\beta}} \\
& =\left(\sigma_{i}^{\underline{\beta}}=\sigma_{\underline{\underline{1}}_{1} \underline{i}_{2}}^{\underline{\beta}}, \sigma_{(1) a_{1}}^{\underline{\beta}}=\sigma_{(1) \underline{\alpha}_{1} \underline{\alpha}_{2}}^{\underline{\beta}}, \ldots,\right. \\
& \left.\sigma_{(p) a_{p}}^{\underline{\beta}}=\sigma_{(p) \underline{\alpha}_{1} \underline{\alpha}_{2}}, \ldots, \sigma_{(z) a_{z}}^{\underline{\beta}}=\sigma_{(z) \underline{\alpha}_{1} \underline{\alpha}_{2}}\right)
\end{aligned}
$$

satisfying conditions

$$
\nabla_{\langle\alpha\rangle}\left(\xi^{\underline{\beta}}+\eta^{\underline{\beta}}\right)=\nabla_{\langle\alpha\rangle} \xi^{\underline{\beta}}+\nabla_{\langle\alpha\rangle} \eta^{\underline{\beta}}, \quad \nabla_{\langle\alpha\rangle}\left(f \xi^{\underline{\beta}}\right)=f \nabla_{\langle\alpha\rangle} \xi^{\underline{\beta}}+\xi^{\underline{\beta}} \nabla_{\langle\alpha\rangle} f
$$

for every $\xi^{\underline{\beta}}, \eta^{\underline{\beta}} \in \sigma^{\underline{\beta}}$ and $f$ being a scalar field on $\mathscr{E}^{\langle z\rangle}$. It is also required that the Leibnitz rule

$$
\left(\nabla_{\langle\alpha\rangle} \zeta_{\underline{\beta}}\right) \eta^{\underline{\beta}}=\nabla_{\langle\alpha\rangle}\left(\zeta_{\underline{\beta}} \eta^{\underline{\beta}}\right)-\zeta_{\underline{\beta}} \nabla_{\langle\alpha\rangle} \eta^{\underline{\beta}}
$$

holds and that $\nabla_{\langle\alpha\rangle}$ be a real operator, that is, it commutes with the operation of complex conjugation:

$$
\overline{\nabla_{\langle\alpha\rangle} \psi_{\underline{\alpha} \underline{\beta} \underline{\gamma} \ldots}}=\nabla_{\langle\alpha\rangle}\left(\bar{\psi}_{\underline{\alpha} \underline{\beta} \underline{\gamma} \ldots}\right) .
$$


We now analyze the question on uniqueness of action on d-spinors of an operator $\nabla_{\langle\alpha\rangle}$ satisfying necessary conditions. Denoting by $\nabla_{\langle\alpha\rangle}^{(1)}$ and $\nabla_{\langle\alpha\rangle}$ two such d-covariant operators, we consider the map

$$
\left(\nabla_{\langle\alpha\rangle}^{(1)}-\nabla_{\langle\alpha\rangle}\right): \sigma^{\underline{\beta}} \longrightarrow \sigma_{\underline{\alpha}_{1}}^{\underline{\beta}} \underline{\alpha}_{2} .
$$

Because the action on a scalar $f$ of both operators $\nabla_{\alpha}^{(1)}$ and $\nabla_{\alpha}$ must be identical, that is,

$$
\nabla_{\langle\alpha\rangle}^{(1)} f=\nabla_{\langle\alpha\rangle} f
$$

the action (6.38) on $f=\omega_{\underline{\beta}} \xi^{\underline{\beta}}$ must be written as

$$
\left(\nabla_{\langle\alpha\rangle}^{(1)}-\nabla_{\langle\alpha\rangle}\right)\left(\omega_{\underline{\beta}} \xi^{\underline{\beta}}\right)=0 .
$$

In consequence, we conclude that there is an element $\Theta_{\underline{\alpha}_{1} \underline{\alpha}_{2} \underline{\beta}}^{\underline{\gamma}} \in \sigma_{\underline{\alpha}_{1} \underline{\alpha}_{2} \underline{\beta}}^{\underline{\gamma}}$ for which

$$
\nabla_{\underline{\alpha}_{1} \underline{\alpha}_{2}}^{(1)} \xi^{\underline{\gamma}}=\nabla_{\underline{\alpha}_{1} \underline{\alpha}_{2}} \xi \underline{\gamma}+\Theta_{\underline{\alpha}_{1} \underline{\alpha}_{2} \underline{\beta}}^{\underline{\underline{\gamma}}} \xi^{\underline{\beta}}, \quad \nabla_{\underline{\alpha}_{1} \underline{\alpha}_{2}}^{(1)} \omega_{\underline{\beta}}=\nabla_{\underline{\alpha}_{1} \underline{\alpha}_{2}} \omega_{\underline{\beta}}-\Theta_{\underline{\alpha}_{1}}^{\underline{\gamma}} \underline{\alpha}_{2} \underline{\beta} \omega_{\underline{\gamma}} .
$$

The action of the operator (6.38) on a d-vector $v^{\langle\beta\rangle}=v \underline{\beta}_{1} \underline{\beta}_{2}$ can be written by using formula (6.41) for both indices $\underline{\beta}_{1}$ and $\underline{\beta}_{2}$ :

$$
\begin{aligned}
& \left(\nabla_{\langle\alpha\rangle}^{(1)}-\nabla_{\langle\alpha\rangle}\right) v \underline{\underline{\beta}}_{1} \underline{\beta}_{2}=\Theta_{\left\langle\alpha \underline{\beta^{\prime}}\right.}^{\underline{\beta}_{1}} v \underline{\underline{\beta}}_{2}+\Theta_{\langle\alpha \underline{\underline{\gamma}}}^{\underline{\beta}_{2}} v \underline{\beta}_{1} \underline{\gamma} \\
& =\left(\Theta \Theta_{\langle\alpha\rangle \underline{\gamma}_{1}}^{\underline{\beta}_{1}} \delta \underline{\underline{\underline{\beta}}}_{2}+\Theta_{\left\langle\alpha \underline{\underline{\gamma}}_{1}\right.}^{\underline{\beta}_{2}} \delta_{\underline{\underline{\beta}}_{2}}^{\underline{\beta}_{1}}\right) v \underline{\underline{\gamma}}_{1} \underline{\underline{\gamma}}_{2} \\
& =Q_{\langle\alpha\rangle\langle y\rangle}^{\langle\beta\rangle} v^{\langle\gamma\rangle} \text {, }
\end{aligned}
$$

where

$$
Q_{\langle\alpha\rangle\langle\gamma\rangle}^{\langle\beta\rangle}=Q_{\underline{\alpha}_{1}}^{\underline{\beta}_{1} \underline{\beta}_{2} \underline{\beta}_{2} \underline{\gamma}_{1} \underline{\gamma}_{2}}=\Theta_{\langle\alpha\rangle \underline{\gamma}_{1}}^{\underline{\beta}_{1}} \delta_{\underline{\underline{z}}_{2}}^{\underline{\beta}_{2}}+\Theta_{\left\langle\alpha \underline{\gamma}_{1}\right.}^{\underline{\beta}_{2}} \delta_{\underline{\underline{z}}_{2}}^{\underline{\beta}_{1}} .
$$

The d-commutator $\nabla_{[\langle\alpha\rangle} \nabla_{\langle\beta\rangle]}$ defines the d-torsion. So, applying operators $\nabla_{[\langle\alpha\rangle}^{(1)} \nabla_{\langle\beta\rangle]}^{(1)}$ and $\nabla_{[\langle\alpha\rangle} \nabla_{\langle\beta\rangle]}$ on $f=\omega_{\underline{\beta}} \xi \underline{\underline{\beta}}$, we can write

$$
T_{\langle\alpha\rangle\langle\beta\rangle}^{(1)\langle\gamma\rangle}-T_{\langle\alpha\rangle\langle\beta\rangle}^{\langle\gamma\rangle}=Q_{\langle\beta\rangle\langle\alpha\rangle}^{\langle\gamma\rangle}-Q_{\langle\alpha\rangle\langle\beta\rangle}^{\langle\gamma\rangle}
$$

with $Q_{\langle\alpha\rangle\langle\beta\rangle}^{\langle\gamma\rangle}$ from (6.43).

The action of operator $\nabla_{\langle\alpha\rangle}^{(1)}$ on d-spinor tensors of type $\chi_{\underline{\alpha}_{1}}^{\underline{\beta}_{1} \underline{\beta}_{2} \underline{\alpha}_{2} \ldots} \underline{\alpha}_{3} \ldots$ must be constructed by using formula (6.41) for every upper index $\underline{\beta}_{1} \underline{\beta}_{2} \ldots$ and formula (6.43) for every lower index $\underline{\alpha}_{1} \underline{\alpha}_{2} \underline{\alpha}_{3} \ldots$

\subsubsection{Infeld-van der Waerden coefficients. Let}

$$
\delta_{\underline{\alpha}}^{\underline{\alpha}}=\left(\delta_{\underline{1}}^{\underline{i}}, \delta_{\underline{2}}^{i}, \ldots, \delta_{\underline{\mathbf{N}(\mathbf{n})}}^{i}, \delta_{\underline{\underline{1}}}^{\underline{a}}, \delta_{\underline{\underline{2}}}^{\underline{a}}, \ldots, \delta_{\underline{\mathbf{N}(\mathbf{m})}}^{i}\right)
$$

be a d-spinor basis. The basis dual to it is denoted as

$$
\delta_{\underline{\alpha}}^{\underline{\alpha}}=\left(\delta_{\underline{i}}^{\underline{1}}, \delta_{\underline{i}}^{\underline{\underline{\alpha}}}, \ldots, \delta_{\underline{\underline{i}}}^{\mathrm{N}(\mathrm{n})}, \delta_{\underline{i}}^{\underline{1}}, \delta_{\underline{\underline{i}}}^{\underline{\underline{\alpha}}}, \ldots, \delta_{\underline{\underline{i}}}^{\mathrm{N}(\mathrm{m})}\right) .
$$


A d-spinor $\kappa^{\underline{\alpha}} \in \sigma^{\underline{\alpha}}$ has components $\kappa^{\underline{\alpha}}=\kappa^{\underline{\alpha}} \delta_{\underline{\alpha}}^{\underline{\alpha}}$. Taking into account that $\delta_{\underline{\alpha}}^{\underline{\alpha}} \delta_{\underline{\beta}}^{\underline{\beta}} \nabla_{\underline{\alpha} \underline{\beta}}=$ $\nabla_{\underline{\alpha} \underline{\beta}}$, we write out the components $\nabla_{\underline{\alpha} \underline{\beta}} \kappa^{\underline{\gamma}}$ in the form

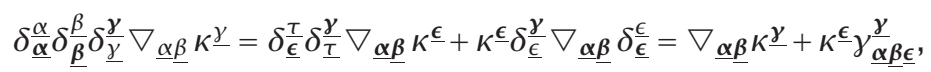

where the coordinate components of the d-spinor connection $\gamma \underline{\underline{\alpha} \underline{\boldsymbol{\gamma}} \underline{\underline{\epsilon}}}$ are defined as

$$
\gamma_{\underline{\alpha} \underline{\beta} \underline{\underline{\epsilon}}}^{\underline{\Rightarrow}} \doteq \delta_{\underline{\tau}}^{\frac{\gamma}{\alpha}} \nabla_{\underline{\alpha} \underline{\beta}} \delta_{\underline{\epsilon}}^{\tau} .
$$

We call the Infeld-van der Waerden d-symbols a set of $\sigma$-objects $\left(\sigma_{\alpha}\right)^{\underline{\alpha} \underline{\beta}}$ parametrized with respect to a coordinate d-spinor basis. Defining

$$
\nabla_{\langle\boldsymbol{\alpha}\rangle}=\left(\sigma_{\langle\boldsymbol{\alpha}\rangle}\right)^{\underline{\boldsymbol{\alpha}} \underline{\beta}} \nabla_{\underline{\boldsymbol{\alpha}} \underline{\beta}},
$$

introducing denotations

$$
\gamma_{\langle\boldsymbol{\alpha}\rangle \underline{\boldsymbol{\tau}}}^{\underline{\boldsymbol{\gamma}}} \doteq \gamma_{\underline{\alpha} \underline{\boldsymbol{\beta}} \underline{\boldsymbol{\tau}}}^{\underline{\gamma}}\left(\sigma_{\langle\alpha \boldsymbol{\alpha}\rangle}\right) \underline{\boldsymbol{\alpha} \underline{\beta}},
$$

and using properties (6.47), we can write the relations

$$
\begin{aligned}
& l_{\langle\alpha\rangle}^{\langle\alpha\rangle} \delta_{\underline{\beta}}^{\underline{\beta}} \nabla_{\langle\alpha\rangle} \kappa^{\underline{\beta}}=\nabla_{\langle\alpha\rangle} \kappa^{\underline{\beta}}+\kappa^{\underline{\delta}} \gamma_{\langle\alpha \boldsymbol{\alpha}\rangle}^{\underline{\beta}}, \\
& l_{\langle\boldsymbol{\alpha}\rangle}^{\langle\alpha\rangle} \delta_{\underline{\beta}}^{\underline{\beta}} \nabla_{\langle\alpha\rangle} \mu_{\underline{\beta}}=\nabla_{\langle\boldsymbol{\alpha}\rangle} \mu_{\underline{\beta}}-\mu_{\underline{\delta}} \gamma_{\langle\boldsymbol{\alpha}\rangle \underline{\boldsymbol{\beta}}}
\end{aligned}
$$

for d-covariant derivations $\nabla_{\underline{\alpha}} \kappa \underline{\underline{\beta}}$ and $\nabla_{\underline{\alpha}} \mu_{\underline{\beta}}$.

We can consider expressions similar to (6.51) for values having both types of d-spinor and d-tensor indices, for instance,

$$
l_{\langle\boldsymbol{\alpha}\rangle}^{\langle\alpha\rangle} l_{\langle\gamma\rangle}^{\langle\gamma\rangle} \delta_{\underline{\delta}}^{\delta} \nabla_{\langle\alpha\rangle} \theta_{\underline{\delta}}^{\langle\gamma\rangle}=\nabla_{\langle\boldsymbol{\alpha}\rangle} \theta_{\underline{\delta}}^{\langle\boldsymbol{\gamma}\rangle}-\theta_{\underline{\epsilon}}^{\langle\boldsymbol{\gamma}\rangle} \gamma_{\langle\boldsymbol{\alpha}\rangle \underline{\delta}}^{\underline{\epsilon}}+\theta_{\underline{\delta}}^{\langle\boldsymbol{\tau}\rangle} \Gamma_{\langle\boldsymbol{\alpha}\rangle\langle\tau\rangle}^{\langle\boldsymbol{\gamma}\rangle}
$$

(we can prove this by a straightforward calculation).

Now we will consider some possible relations between components of d-connections $\gamma \frac{\boldsymbol{\epsilon}}{\langle\boldsymbol{\alpha}\rangle \underline{\delta}}$ and $\Gamma_{\langle\boldsymbol{\alpha}\rangle\langle\tau\rangle}^{\langle\boldsymbol{\gamma}\rangle}$ and derivations of $\left(\sigma_{\langle\boldsymbol{\alpha}\rangle}\right) \underline{\boldsymbol{\alpha}} \underline{\underline{\beta}}$. We can write

$$
\begin{aligned}
& \Gamma_{\langle\boldsymbol{\beta}\rangle\langle\gamma\rangle}^{\langle\boldsymbol{\alpha}\rangle}=l_{\langle\alpha\rangle}^{\langle\boldsymbol{\alpha}\rangle} \nabla_{\langle\boldsymbol{\gamma}\rangle} l_{\langle\boldsymbol{\beta}\rangle}^{\langle\alpha\rangle}=l_{\langle\alpha\rangle}^{\langle\boldsymbol{\alpha}\rangle} \nabla_{\langle\boldsymbol{\gamma}\rangle}\left(\sigma_{\langle\boldsymbol{\beta}\rangle}\right)^{\underline{\epsilon} \underline{\underline{T}}} l_{\langle\alpha\rangle}^{\langle\boldsymbol{\alpha}\rangle} \nabla_{\langle\boldsymbol{\gamma}\rangle}\left(\left(\sigma_{\langle\boldsymbol{\beta}\rangle}\right)^{\underline{\epsilon} \boldsymbol{T}} \delta_{\underline{\epsilon}}^{\underline{\epsilon}} \delta_{\underline{\underline{T}}}^{\underline{\boldsymbol{T}}}\right)
\end{aligned}
$$

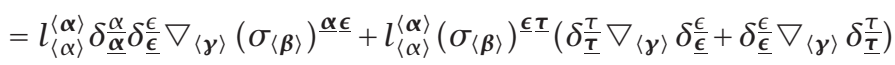

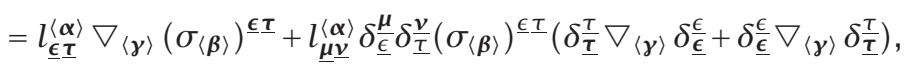

where $l_{\langle\alpha\rangle}^{\langle\boldsymbol{\alpha}\rangle}=\left(\sigma_{\underline{\boldsymbol{\epsilon}} \boldsymbol{\tau}}\right)^{\langle\boldsymbol{\alpha}\rangle}$, from which follows

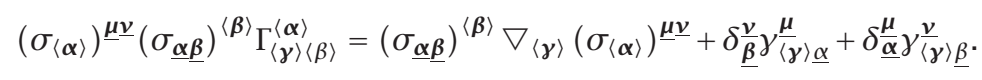

Connecting the last expressions on $\underline{\boldsymbol{\beta}}$ and $\underline{\boldsymbol{\nu}}$ and using an orthonormalized d-spinor basis when $\gamma_{\langle\boldsymbol{\gamma}\rangle \underline{\beta}}^{\underline{\beta}}=0$ (a consequence from (6.48)), we have

$$
\gamma^{\underline{\langle\boldsymbol{\gamma}\rangle \underline{\alpha}}}=\frac{1}{N(n)+N\left(m_{1}\right)+\cdots+N\left(m_{z}\right)}\left(\Gamma_{\langle\bar{\gamma}\rangle \underline{\boldsymbol{\alpha}} \underline{\beta}}-\left(\sigma_{\underline{\alpha} \underline{\beta}}\right)^{\langle\boldsymbol{\beta}\rangle} \nabla_{\langle\gamma\rangle}\left(\sigma_{\langle\boldsymbol{\beta}\rangle}\right) \underline{\mu} \underline{\beta}\right),
$$


where

$$
\Gamma_{\langle\boldsymbol{\gamma}\rangle \underline{\underline{\alpha}} \underline{\beta}}=\left(\sigma_{\langle\boldsymbol{\alpha}\rangle}\right)^{\underline{\mu} \underline{\beta}}\left(\sigma_{\underline{\alpha} \underline{\beta}}\right)^{\boldsymbol{\beta}} \Gamma_{\langle\boldsymbol{\gamma}\rangle\langle\beta\rangle}^{\langle\boldsymbol{\alpha}\rangle} .
$$

We also note here that, for instance, for the canonical and Berwald connections and Christoffel d-symbols, we can express d-spinor connection (6.56) through corresponding locally adapted derivations of components of metric and N-connection by introducing corresponding coefficients instead of $\Gamma_{\langle\boldsymbol{\gamma}\rangle\langle\beta\rangle}^{\langle\boldsymbol{\alpha}\rangle}$ in (6.56) and then in (6.55).

6.2.3. d-spinors of ha-space curvature and torsion. The d-tensor indices of the commutator $\Delta_{\langle\alpha\rangle\langle\beta\rangle}$ can be transformed into d-spinor ones:

$$
\square_{\underline{\alpha} \underline{\beta}}=\left(\sigma^{\langle\alpha\rangle\langle\beta\rangle}\right)_{\underline{\alpha} \underline{\beta}} \Delta_{\alpha \beta}=\left(\square_{\underline{i}}, \square_{\underline{a}} \underline{b}\right)=\left(\square_{\underline{i}}, \square_{\underline{a}_{1} \underline{b}_{1}}, \ldots, \square_{\underline{a}_{p} \underline{b}_{p}}, \ldots, \square_{\underline{a}_{z}} \underline{b}_{z}\right),
$$

with $\mathrm{h}$ - and $\mathrm{v}_{p}$-components,

$$
\square_{\underline{i j}}=\left(\sigma^{\langle\alpha\rangle\langle\beta\rangle}\right)_{\underline{i j} \underline{j}} \Delta\langle\alpha\rangle\langle\beta\rangle, \quad \square_{\underline{a} \underline{b}}=\left(\sigma^{\langle\alpha\rangle\langle\beta\rangle}\right)_{\underline{a} \underline{b}} \Delta\langle\alpha\rangle\langle\beta\rangle,
$$

being symmetric or antisymmetric depending on the corresponding values of dimensions $n$ and $m_{p}$ (see eight-fold parametrizations). Considering the actions of operator (6.57) on d-spinors $\pi \underline{\gamma}$ and $\mu_{\underline{\gamma}}$, we introduce the d-spinor curvature $X_{\underline{\delta}}^{\underline{\gamma} \alpha \underline{\beta}}$ as to satisfy equations

$$
\square_{\underline{\alpha} \underline{\beta}} \pi \underline{\gamma}=X_{\underline{\delta} \underline{\alpha} \underline{\beta}}^{\underline{\gamma}} \pi \underline{\delta}, \quad \square_{\underline{\alpha} \underline{\beta}} \mu_{\underline{\gamma}}=X_{\underline{\underline{\gamma}} \underline{\alpha} \underline{\beta}}^{\underline{\delta}} \mu_{\underline{\delta}} .
$$

The gravitational d-spinor $\Psi_{\underline{\alpha} \underline{\beta}} \underline{\gamma} \underline{\delta}$ is defined by a corresponding symmetrization of dspinor indices:

$$
\Psi_{\underline{\alpha} \underline{\beta} \underline{\gamma} \underline{\delta}}=X_{(\underline{\alpha}|\underline{\beta}| \underline{\gamma} \underline{\delta})} .
$$

We note that d-spinor tensors $X_{\underline{\delta} \underline{\gamma} \underline{\gamma}}$ and $\Psi_{\underline{\alpha} \underline{\beta}} \underline{\gamma} \underline{\delta}$ are transformed into similar 2-spinor objects on locally isotropic spaces $[6 \overline{1}, 62,63]$ if we consider vanishing of the $\mathrm{N}$-connection structure and a limit to a locally isotropic space.

Putting $\delta \underline{\underline{\gamma}}$ instead of $\mu_{\underline{\gamma}}$ in (6.59) and using (6.60), we can express, respectively, the curvature and gravitational d-spinors as

$$
X_{\underline{\gamma} \underline{\delta} \underline{\alpha} \underline{\beta}}=\delta_{\underline{\delta} \underline{\boldsymbol{\tau}}}^{\square \underline{\alpha} \underline{\beta}} \delta_{\underline{\gamma}}^{\underline{\boldsymbol{\tau}}}, \quad \Psi_{\underline{\gamma} \underline{\delta} \underline{\alpha} \underline{\beta}}=\delta_{\underline{\delta} \underline{\boldsymbol{\tau}}} \square\left(\underline{\alpha} \underline{\beta} \delta_{\underline{\gamma}}{ }^{\underline{\boldsymbol{\tau}}} .\right.
$$

The d-spinor torsion $T_{\underline{\alpha} \underline{\beta}}^{\underline{\gamma}} \underline{\gamma}_{2}$ is defined similarly as for d-tensors by using the d-spinor commutator (6.57) and equations

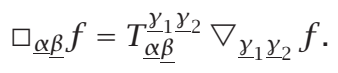

The d-spinor components $R_{\underline{\gamma}_{1}} \underline{\delta}_{1} \underline{\delta}_{2} \underline{\alpha} \underline{\beta}$ of the curvature d-tensor $R_{\gamma \alpha \beta}^{\delta}$ can be computed by using relations (6.56), (6.57), and (6.60) as to satisfy the equations

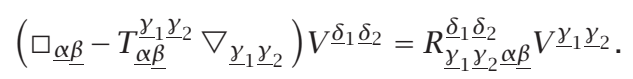


Here d-vector $V \underline{\underline{\gamma}}_{1} \underline{\underline{\gamma}}_{2}$ is considered as a product of d-spinors, that is, $V_{\underline{\gamma}_{1}} \underline{\underline{\gamma}}_{2}=\boldsymbol{\nu} \underline{\underline{\gamma}}_{1} \boldsymbol{\mu}^{\underline{\gamma_{2}}}$. We find

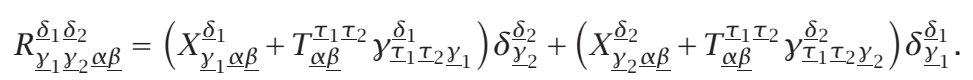

It is convenient to use this d-spinor expression for the curvature d-tensor

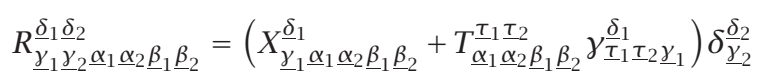

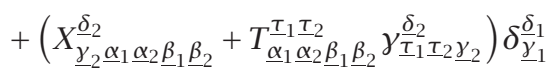

in order to get the d-spinor components of the Ricci d-tensor

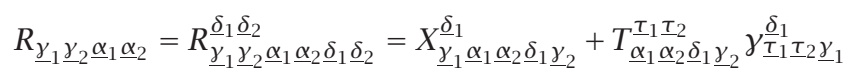

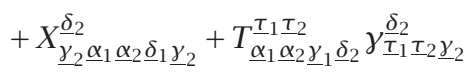

and the following d-spinor decomposition of the scalar curvature:

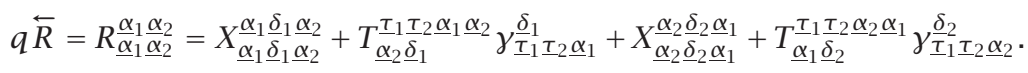

Using (6.66) and (6.67), see details in [61,62, 63], we define the d-spinor components of the Einstein and $\Phi_{\langle\alpha\rangle\langle\beta\rangle}$ d-tensors:

$$
\begin{aligned}
& \overleftarrow{G}_{\langle\gamma\rangle\langle\alpha\rangle}=\overleftarrow{G}_{\underline{\gamma}_{1} \underline{\gamma}_{2} \underline{\alpha}_{1} \underline{\alpha}_{2}}=X_{\underline{\gamma}_{1} \underline{\alpha}_{1} \underline{\alpha}_{2} \underline{\delta}_{1} \underline{\gamma}_{2}}^{\underline{\delta}_{1}}+T_{\underline{\alpha}_{1} \underline{\alpha}_{2} \underline{\boldsymbol{\alpha}}_{1} \underline{\boldsymbol{\tau}}_{2} \underline{\gamma}_{2}} \gamma_{\underline{\underline{\tau}}_{1} \underline{\underline{\tau}}_{2} \underline{\gamma}_{1}}
\end{aligned}
$$

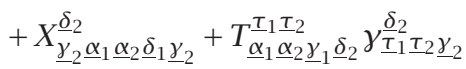

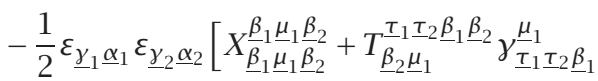

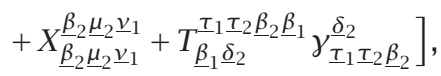

$$
\begin{aligned}
& \Phi_{\langle\gamma\rangle\langle\alpha\rangle}=\Phi_{\underline{\gamma}_{1} \underline{\gamma}_{2} \underline{\alpha}_{1} \underline{\alpha}_{2}}=\frac{1}{2\left(n+m_{1}+\cdots+m_{z}\right)}
\end{aligned}
$$

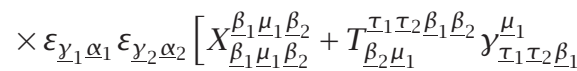

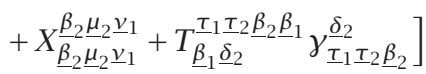

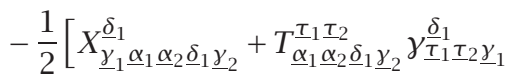

$$
\begin{aligned}
& \left.+X_{\underline{\gamma}_{2} \underline{\alpha}_{1} \underline{\alpha}_{2} \underline{\delta}_{1} \underline{\gamma}_{2}}^{\underline{\delta}_{2}}+T_{\underline{\alpha}_{1} \underline{\alpha}_{2} \underline{\gamma}_{1} \underline{\boldsymbol{\delta}}_{2}}^{\underline{\boldsymbol{\delta}}_{1} \underline{\underline{\tau}}_{2}} \underline{\underline{\underline{\tau}}}_{1} \underline{\underline{\tau}}_{2} \underline{\gamma}_{2}\right] .
\end{aligned}
$$

We omit this calculus in this work.

ACKNOWLEDGMENTS. The work of S. Vacaru is supported by a NATO/Portugal fellowship at CENTRA, Instituto Superior Tecnico, Lisbon. The author is grateful to R. Ablamowicz, John Ryan, and B. Fauser for collaboration and support of his participation at "The 6th International Conference on Clifford Algebras," Cookeville, Tennessee, USA (May, 20-25, 2002). He would like to thank J. P. S. Lemos, R. Miron, M. Anastasiei, and P. Stavrinos for hospitality and support. 


\section{REFERENCES}

[1] M. C. B. Abdalla, M. A. De Andrade, M. A. Santos, and I. V. Vancea, On the symplectic two-form of gravity in terms of Dirac eigenvalues, Phys. Lett. B 548 (2002), no. 1-2, 88-91.

[2] M. Anastasiei, Structures spinorielles sur les variétés hilbertiennes, C. R. Acad. Sci. Paris Sér. A-B 284 (1977), no. 16, A943-A946 (French).

[3] F. Ardalan, H. Arfaei, M. R. Garousi, and A. Ghodsi, Gravity on noncommutative D-branes, Internat. J. Modern Phys. A 18 (2003), no. 7, 1051-1066.

[4] G. S. Asanov and S. F. Ponomarenko, Finsler Bundle on Space-Time. Associated Gauge Fields and Connections, Stiinca, Kishinev, 1988.

[5] A. Ashtekar, New Hamiltonian formulation of general relativity, Phys. Rev. D (3) 36 (1987), no. 6, 1587-1602.

[6] M. F. Atiyah, R. Bott, and A. Shapiro, Clifford modules, Topology 3 (1964), suppl. 1, 3-38.

[7] I. M. Benn and R. W. Tucker, An Introduction to Spinors and Geometry with Applications in Physics, Adam Hilger, Bristol, 1989.

[8] M. A. Cardella and D. Zanon, Noncommutative deformation of four-dimensional Einstein gravity, Classical Quantum Gravity 20 (2003), no. 8, L95-L103.

[9] E. Cartan, Exposés de Géométrie, Series Actualités Scientifiques et Industrielles, vol. 79, 1936, reprinted, Hermann, Paris, 1971.

[10] _ _ Leçons sur la Théorie des Spineurs Tome I: Les Spineurs de l'Espace á $n>3$ Dimensions. Les Spineurs en Gèometrie Reimannienne, Hermann, Paris, 1938.

[11]_ The Theory of Spinors, Dover Publications, New York, 1966.

[12] A. H. Chamseddine, G. Felder, and J. Fröhlich, Gravity in noncommutative geometry, Comm. Math. Phys. 155 (1993), no. 1, 205-217.

[13] C. Chevalley, The Construction and Study of Certain Important Algebras, The Mathematical Society of Japan, Tokyo, 1955.

[14] W. K. Clifford, A preliminary sketch of biquaternions, Proc. London Math. Soc. 4 (1876), 381-395.

[15] A. Connes, Noncommutative Geometry, Academic Press, California, 1994.

[16] A. Crumeyrolle, Structures spinorielles, Ann. Inst. H. Poincaré Sect. A (N.S.) 11 (1969), 19-55 (French).

[17] _ G Groupes de spinorialité, Ann. Inst. H. Poincaré Sect. A (N.S.) 14 (1971), 309-323 (French).

[18] P. Deligne, P. Etingof, D. S. Freed, L. C. Jeffrey, D. Kazhdan, J. W. Morgan, D. R. Morrison, and E. Witten (eds.), Quantum Fields and Strings: A Course for Mathematicians. Vol. 1, 2, American Mathematical Society, Rhode Island, 1999.

[19] T. Dereli, M. Önder, J. Schray, R. W. Tucker, and C. Wang, Non-Riemannian gravity and the Einstein-Proca system, Classical Quantum Gravity 13 (1996), no. 8, L103-L109.

[20] P. A. M. Dirac, The quantum theory of the electron, I, II, Proc. Roy. Soc. London Ser. A 117 (1928), 610-624.

[21] V. Fock, Geometrisierung der Diracschen theorie des elektrons, Z. Physik 57 (1929), 261277 (German).

[22] H. García-Compeán, O. Obregón, C. Ramírez, and M. Sabido, Noncommutative self-dual gravity, Phys. Rev. D (3) 68 (2003), no. 4, 044015, 8.

[23] F. W. Hehl, J. D. McCrea, E. W. Mielke, and Y. Ne'eman, Metric-affine gauge theory of gravity: field equations, Noether identities, world spinors, and breaking of dilation invariance, Phys. Rep. 258 (1995), no. 1-2, 1-171.

[24] F. W. Hehl, P. von der Heyde, and G. D. Kerlick, General relativity with spin and torsion and its deviations from Einstein's theory, Phys. Rev. D (3) 10 (1974), 1066-1069.

[25] F. W. Hehl, P. von der Heyde, G. D. Kerlick, and J. M. Nester, General relativity with spin and torsion: foundations and prospect, Rev. Modern Phys. 48 (1976), no. 3, 393-416.

[26] J. Hladik, Les Spineurs en Physique, Masson, Paris, 1996. 
[27] _ Spinors in Physics, Graduate Texts in Contemporary Physics, Springer-Verlag, New York, 1999.

[28] M. Karoubi, K-Theory, Springer-Verlag, Berlin, 1978.

[29] M. Kawaguchi, Jets semi-holonomes et tenseurs non-symétriques d'ordre supérieur. I, J. Fac. Sci. Hokkaido Univ. Ser. I 16 (1962), 193-213 (French).

[30] J. Kern, Lagrange geometry, Arch. Math. (Basel) 25 (1974), 438-443.

[31] C. P. Luehr and M. Rosenbaum, Spinor connections in general relativity, J. Mathematical Phys. 15 (1974), 1120-1137.

[32] J. Madore, An Introduction to Noncommutative Differential Geometry and Its Physical Applications, London Mathematical Society Lecture Note Series, vol. 257, Cambridge University Press, Cambridge, 1999.

[33] S. Majid, Foundations of Quantum Group Theory, Cambridge University Press, Cambridge, 1995.

[34] Conceptual issues for noncommutative gravity on algebras and finite sets, Internat. J. Modern Phys. B 14 (2000), no. 22-23, 2427-2449.

[35]___ Meaning of noncommutative geometry and the Planck-scale quantum group, Towards Quantum Gravity (Polanica, 1999), Lecture Notes in Phys., vol. 541, SpringerVerlag, Berlin, 2000, pp. 227-276.

[36] , Non-commutative geometry and quantum groups, R. Soc. Lond. Philos. Trans. Ser. A Math. Phys. Eng. Sci. 358 (2000), no. 1765, 89-109.

[37] _ A Quantum Groups Primer, London Mathematical Society Lecture Note Series, vol. 292, Cambridge University Press, Cambridge, 2002.

[38] R. Miron, A Lagrangian theory of relativity, An. Şti. Univ. “Al. I. Cuza” Iaşi Secț. I a Mat. 32 (1986), no. 2, 37-62.

[39] _ A Lagrangian theory of relativity. II, An. Şti. Univ. "Al. I. Cuza” Iaşi Secț. I a Mat. 32 (1986), no. 3, 7-16.

[40] Cartan spaces in a new point of view by considering them as duals of Finsler spaces, Tensor (N.S.) 46 (1987), 329-334.

[41]_ Hamilton geometry, Univ. Timişoara, Sem. Mecanica 3 (1987), 54.

[42] _ The geometry of Cartan spaces, Progr. Math. (Varanasi) 22 (1988), no. 1-2, 1-38.

[43] _ Sur la geometrie de espaces Hamilton [The geometry of Hamilton spaces], C. R. Acad. Sci. Paris Sér. I Math. 306 (1988), 195-189 (French).

[44] _ _ Hamilton geometry, An. Şti. Univ. “Al. I. Cuza” Iaşi Secț. I a Mat. 35 (1989), no. 1, 33-67.

[45] _ The Geometry of Higher-Order Lagrange Spaces Applications to Mechanics and Physics, Fundamental Theories of Physics, vol. 82, Kluwer Academic Publishers, Dordrecht, 1997.

[46] R. Miron and M. Anastasiei, Fibrate Vectoriale. Spații Lagrange. Aplicații în Teoria Relativității [Vector Bundles. Lagrange Spaces. Applications to the Theory of Relativity], Editura Academiei Republicii Socialiste România, Bucharest, 1987.

[47] _ The Geometry of Lagrange Spaces: Theory and Applications, Fundamental Theories of Physics, vol. 59, Kluwer Academic Publishers, Dordrecht, 1994.

[48] _ _ Vector Bundles and Lagrange Spaces with Applications to Relativity, Balkan Society of Geometers Monographs and Textbooks, vol. 1, Geometry Balkan Press, Bucharest, 1997.

[49] R. Miron and G. Atanasiu, Higher order Lagrange spaces, Rev. Roumaine Math. Pures Appl. 41 (1996), no. 3-4, 251-262.

[50] _ Prolongation of Riemannian, Finslerian and Lagrangian structures, Rev. Roumaine Math. Pures Appl. 41 (1996), no. 3-4, 237-249.

[51] R. Miron and Gh. Atanasiu, Differential geometry of the $k$-osculator bundle, Rev. Roumaine Math. Pures Appl. 41 (1996), no. 3-4, 205-236. 
[52] R. Miron, D. Hrimiuc, H. Shimada, and S. V. Sabau, The Geometry of Hamilton and Lagrange Spaces, Fundamental Theories of Physics, vol. 118, Kluwer Academic Publishers, Dordrecht, 2001.

[53] J. W. Moffat, Noncommutative quantum gravity, Phys. Lett. B 491 (2000), no. 3-4, 345352.

[54] M. Morand, Géométrie spinorielle. Collection d'Ouvrages de Mathématiques à l'Usage des Physiciens, Masson et Cie, Paris, 1973 (French).

[55] H. Nishino and S. Rajpoot, Noncommutative nonlinear supersymmetry, preprint, 2002, http://arXiv.org/abs/hep-th/0212329.

[56] Yu. N. Obukhov, E. J. Vlachynsky, W. Esser, and F. W. Hehl, Effective Einstein theory from metric-affine gravity models via irreducible decompositions, Phys. Rev. D (3) 56 (1997), no. 12, 7769-7778.

[57] T. Ono and Y. Takano, The differential geometry of spaces whose metric tensor depends on spinor variables and the theory of spinor gauge fields. II, Tensor (N.S.) 49 (1990), no. $1,65-80$.

[58] _ - The differential geometry of spaces whose metric tensor depends on spinor variables and the theory of spinor gauge fields. III, Tensor (N.S.) 49 (1990), no. 3, 253258.

[59]___ Remarks on the spinor gauge field theory, Tensor (N.S.) 52 (1993), no. 1, 56-60.

[60] W. Pauli, Zur Quantenmechanik des magnetischen Elektrons, Z. Physik 43 (1927), 601-623 (German).

[61] R. Penrose, Structure of space-time, Battelle Rencontres (C. M. DeWitt and J. A. Wheeler, eds.), 1967 Lectures in Mathematics and Physics, Benjamin, New York, 1968, pp. 121-235.

[62] R. Penrose and W. Rindler, Spinors and Space-Time. Vol. 1, Two-Spinor Calculus and Relativistic Fields, Cambridge Monographs on Mathematical Physics, Cambridge University Press, Cambridge, 1984.

[63] _ Spinors and Space-Time. Vol. 2, Spinor and Twistor Methods in Space-Time Geometry, Cambridge Monographs on Mathematical Physics, Cambridge University Press, Cambridge, 1986.

[64] J. Polchinski, String Theory. Vol. I, Cambridge Monographs on Mathematical Physics, Cambridge University Press, Cambridge, 1998.

[65] _ String Theory. Vol. II, Cambridge Monographs on Mathematical Physics, Cambridge University Press, Cambridge, 1998.

[66] H. Rund, The Differential Geometry of Finsler Spaces, Die Grundlehren der Mathematischen Wissenschaften, vol. 101, Springer-Verlag, Berlin, 1959.

[67] V. Sahakian, Transcribing spacetime data into matrices, J. High Energy Phys. 0106 (2001), 037.

[68] E. Schrödinger, Diracsches elektron im Schwerfeld, Sitzungsb. Akad. Physik 57 (1929), 261-277 (German).

[69] P. Stavrinos, V. Balan, P. Manouselis, and N. Prezas, Field equations in spaces with $g_{\mu \nu}(x, \xi, \bar{\xi})$ metric. Generalized conformally flat spaces, Rep. Math. Phys. 37 (1996), no. 2, 163-175.

[70] P. Stavrinos, V. Balan, N. Prezas, and P. Manouselis, Spinor bundle of order two on the internal deformed system, An. Şti. Univ. “Al. I. Cuza” Iaşi Secț. I a Mat. 43 (1997), no. $1,51-62$.

[71] P. Stavrinos and S. Koutroubis, Curvature and Lorentz transformations of spaces whose metric tensor depends on vector and spinor variables, Tensor (N.S.) 55 (1994), no. 1, 11-19.

[72] P. Stavrinos and P. Manouselis, Nonlocalized field theory over spinor bundles: Poincaré gravity and Yang-Mills fields, Rep. Math. Phys. 36 (1995), no. 2-3, 439-452. 
[73] Y. Takano, The differential geometry of spaces whose metric tensor depends on spinor variables and the theory of spinor gauge fields, Tensor (N.S.) 40 (1983), no. 3, 249260.

[74] Y. Takano and T. Ono, The differential geometry of spaces whose metric tensor depends on vector and spinor variables and the theory of gauge fields, Tensor (N.S.) 49 (1990), no. 3, 269-279.

[75] S. I. Vacaru, Generalized Finsler geometry in Einstein, string and metric-affine gravity, preprint, 2003, http://arXiv.org/abs/hep-th/0310132.

[76] _ Locally anisotropic interactions: I. Nonlinear connections in higher order anisotropic superspaces, preprint, 1996, http://arXiv.org/abs/hep-th/9607194. , Locally anisotropic interactions: II. Torsions and curvatures of higher order

anisotropic superspaces, preprint, 1996, http://arXiv.org/abs/hep-th/9607195.
, Locally anisotropic interactions: III. Higher order anisotropic supergravity, preprint, 1996, http://arXiv.org/abs/hep-th/9607196.

] _ (Non) commutative Finsler geometry from string/M-theory, preprint, 2002, http://arXiv.org/abs/hep-th/0211068.

[_ Non Nommutative Finsler geometry, gauge fields and gravity, preprint, 2002, http://arXiv.org/abs/math-ph/0205023.

1 _ Nonlinear connections in superbundles and locally anisotropic supergravity, preprint, 1996, http://arXiv.org/abs/gr-qc/9604016.

2] Spinors in higher dimensional and locally anisotropic spaces, preprint, 1996, http://arXiv.org/abs/gr-qc/9604015.

3]___ Spinors, nonlinear connections and nearly autoparallel maps of generalized Finsler spaces, preprint, 1996, http://arXiv.org/abs/dg-ga/9609004.

_ Stochastic processes and diffusion on spaces with local anisotropy, preprint, 1996, http://arXiv.org/abs/gr-qc/9604014.

[86] http://arXiv.org/abs/gr-qc/9905053. Univ. Ser. III Fiz. Astronom, 28 (1987), no. 5, 5-11 (Russian).

[87] __ Stochastic calculus on generalized Lagrange spaces, The Program of the Iasi Academic Days, Academia Romana, Filiala Iasi, Iasi, 1994, p. 30.

[88] _ Clifford structures and spinors on spaces with local anisotropy, Izv. Akad. Nauk Respub. Moldova Fiz. Tekhn. (1995), no. 3, 53-62.

[89] _ Spinor structures and nonlinear connections in vector bundles, generalized Lagrange and Finsler spaces, J. Math. Phys. 37 (1996), no. 1, 508-523.

[90] __ Locally anisotropic gravity and strings, Ann. Physics 256 (1997), no. 1, 39-61.

[91] _ Superstrings in higher order extensions of Finsler superspaces, Nuclear Phys. B 494 (1997), no. 3, 590-656.

[92] _ Interactions, Strings and Isotopies in Higher Order Anisotropic Superspaces, Hadronic Press Monographs in Mathematics, Hadronic Press, Florida, 1998.

[93] _ Spinors and field interactions in higher order anisotropic spaces, J. High Energy Phys. 9809 (1998), 20.

[94]__ Anholonomic soliton-dilaton and black hole solutions in general relativity, J. High Energy Phys. 0104 (2001), 48.

[95]___ Gauge and Einstein gravity from non-abelian gauge models on noncommutative spaces, Phys. Lett. B 498 (2001), no. 1-2, 74-82.

[96]_L__ Locally anisotropic kinetic processes and thermodynamics in curved spaces, Ann. Physics 290 (2001), no. 2, 83-123.

[97] _ Horizons and geodesics of black ellipsoids, Internat. J. Modern Phys. D 12 (2003), no. 3, 479-494.

[98] S. I. Vacaru and H. Dehnen, Locally anisotropic structures and nonlinear connections in Einstein and gauge gravity, Gen. Relativity Gravitation 35 (2003), no. 2, 209-250. 
[99] S. I. Vacaru and E. Gaburov, Noncommutative symmetries and stability of black ellipsoids in metric-affine and string gravity, preprint, 2003, http://arXiv.org/abs/ hep-th/0310134.

[100] S. I. Vacaru, E. Gaburov, and D. Gontsa, A method of constructing off-diagonal solutions in metric-affine and string gravity, preprint, 2003, http://arXiv.org/abs/ hep-th/0310133.

[101] S. I. Vacaru and S. V. Ostaf, Twistors and nearly autoparallel maps, Rep. Math. Phys. 37 (1996), no. 3, 309-324.

[102] S. I. Vacaru and F. C. Popa, Dirac spinor waves and solitons in anisotropic Taub-NUT spaces, Classical Quantum Gravity 18 (2001), no. 22, 4921-4938.

[103] S. I. Vacaru and D. Singleton, Ellipsoidal, cylindrical, bipolar and toroidal wormholes in 5D gravity, J. Math. Phys. 43 (2002), no. 5, 2486-2504.

[104] _ Warped solitonic deformations and propagation of black holes in 5D vacuum gravity, Classical Quantum Gravity 19 (2002), no. 14, 3583-3601.

[105] S. I. Vacaru, D. Singleton, V. A. Boțan, and D. A. Doțenco, Locally anisotropic wormholes and flux tubes in 5D gravity, Phys. Lett. B 519 (2001), no. 3-4, 249-259.

[106] S. I. Vacaru and P. Stavrinos, Spinors and Space-Time Anisotropy, Athens University Press, Athens, 2002.

[107] S. I. Vacaru, P. Stavrinos, and E. Gaburov, Anholonomic triads and new classes of $(2+1)$-dimensional black hole solutions, preprint, 2001, http://arXiv.org/abs/ gr-qc/0106068.

[108] S. I. Vacaru, P. Stavrinos, and D. Gontsa, Anholonomic frames and thermodynamic geometry of 3D black holes, preprint, 2001, http://arXiv.org/abs/gr-qc/0106069.

[109] S. I. Vacaru, P. Stavrinos, and N. Vicol, Spinors in Hamilton and Cartan spaces, Contribution at International Conference on "Finsler, Lagrange and Hamilton Spaces", "Al. I. Cuza" University of Iaşi, Iaşi, 2001.

[110] S. I. Vacaru and O. Tintareanu-Mircea, Anholonomic frames, generalized Killing equations, and anisotropic Taub-NUT spinning spaces, Nuclear Phys. B 626 (2002), no. 1-2, 239-264.

[111] S. I. Vacaru and N. Vicol, Nonlinear connections and dual Clifford structures, Contribution to the 6th International Conference on Clifford Algebras, Tennessee, 2002.

[112] I. V. Vancea, Observables of Euclidean supergravity, Phys. Rev. Lett. 79 (1997), no. 17, 3121-3124.

[113] H. Weyl, Elektron und Gravitation, Z. f. Physik 56 (1929), 330-352 (German).

Sergiu I. Vacaru: Centro Multidisciplinar de Astrofisica - CENTRA, Departamento de Fisica, Instituto Superior Tecnico, Lisboa 1049-001, Portugal

E-mail address: vacaru@fisica.ist.ut1.pt

Nadejda A. Vicol: Faculty of Mathematics and Informatics, State University of Moldova, Chişinău, MD 2009, Republic of Moldova

E-mail address: nadejda_vicol@yahoo.com 


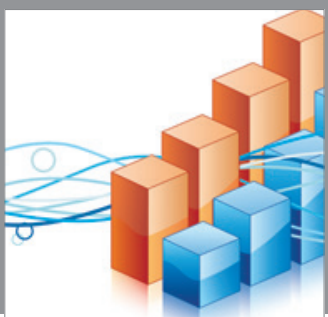

Advances in

Operations Research

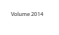

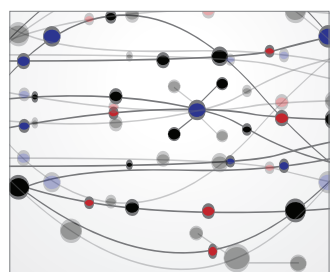

\section{The Scientific} World Journal
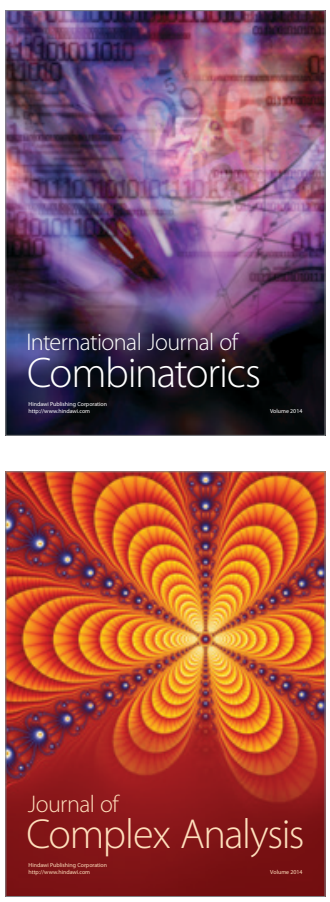

International Journal of

Mathematics and

Mathematical

Sciences
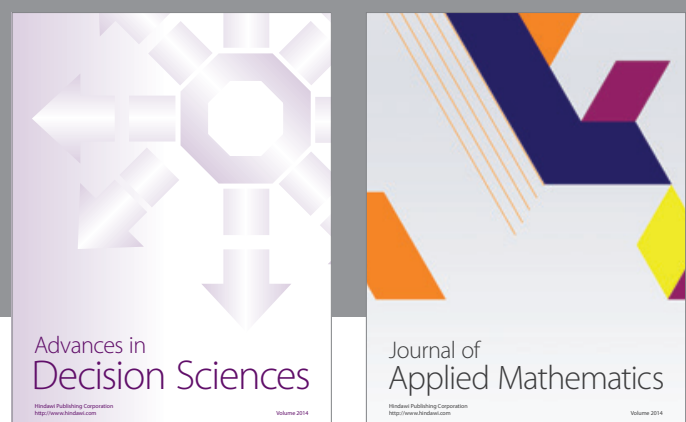

Journal of

Applied Mathematics
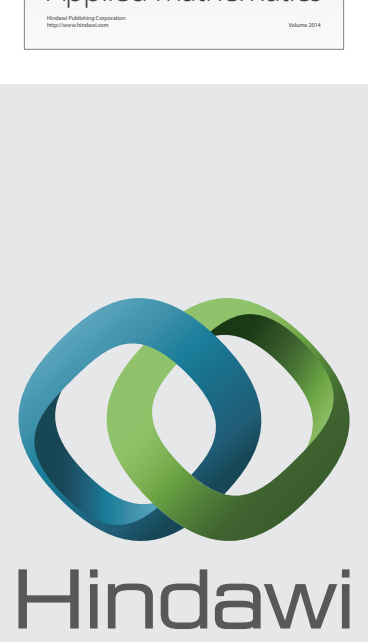

Submit your manuscripts at http://www.hindawi.com
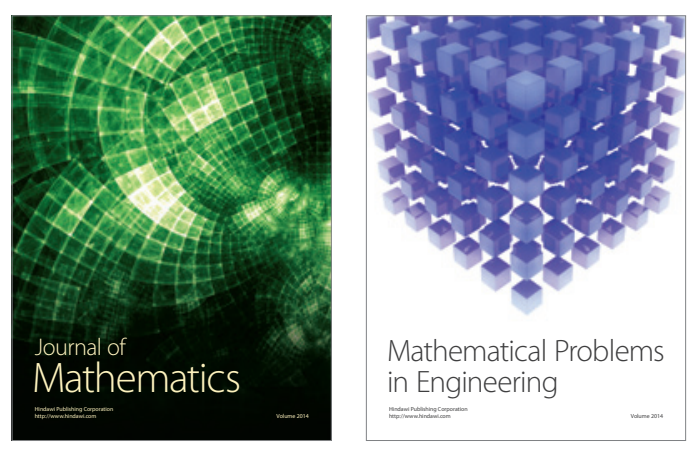

Mathematical Problems in Engineering
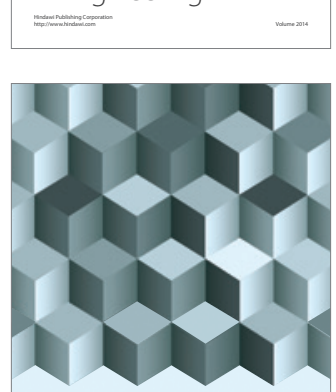

Journal of

Function Spaces
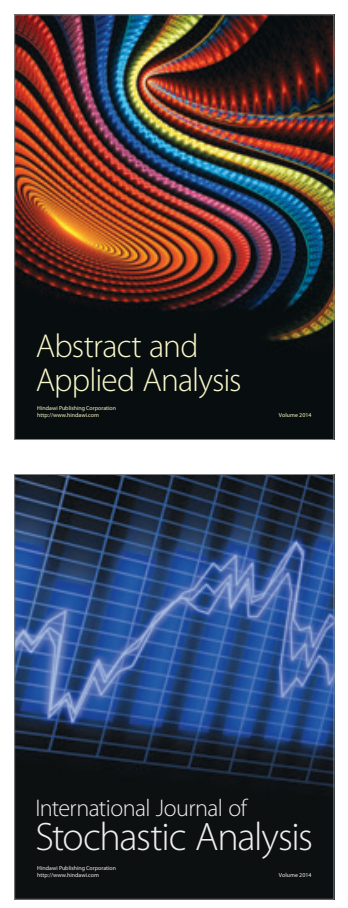

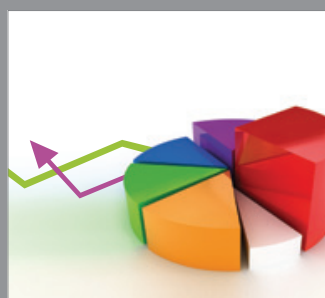

ournal of

Probability and Statistics

Promensencen
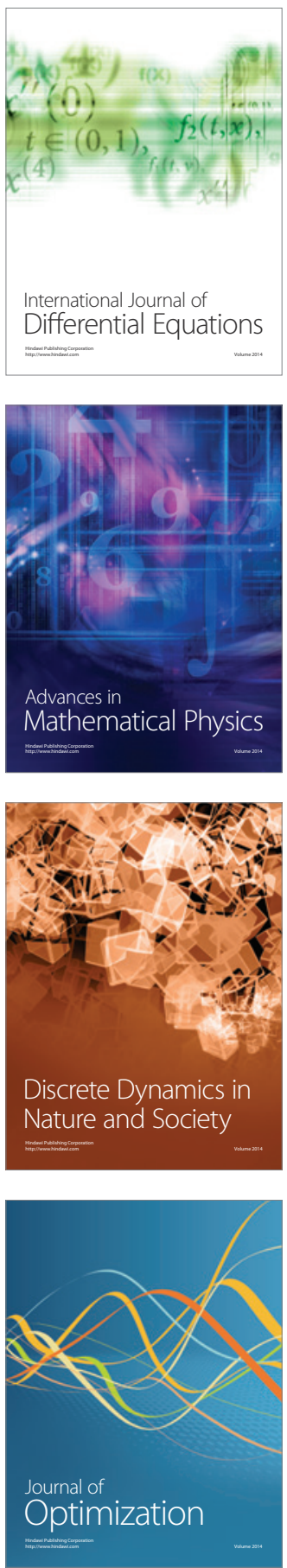